2020-07-24

\title{
A Prospective Cohort Study of Metabolic Syndrome and Endometrial Cancer Survival
}

Kokts-Porietis, Renee L.

Kokts-Porietis, R. L. (2020). A Prospective Cohort Study of Metabolic Syndrome and

Endometrial Cancer Survival (Unpublished master's thesis). University of Calgary, Calgary, AB.

http://hdl.handle.net/1880/112340

Downloaded from PRISM Repository, University of Calgary 


\title{
UNIVERSITY OF CALGARY
}

A Prospective Cohort Study of Metabolic Syndrome and Endometrial Cancer Survival

by

Renee L. Kokts-Porietis

\begin{abstract}
A THESIS
SUBMITTED TO THE FACULTY OF GRADUATE STUDIES

IN PARTIAL FULFILMENT OF THE REQUIREMENTS FOR THE DEGREE OF MASTER OF SCIENCE
\end{abstract}

GRADUATE PROGRAM IN COMMUNITY HEALTH SCIENCES

CALGARY, ALBERTA

JULY, 2020

(C) Renee L. Kokts-Porietis 2020 


\begin{abstract}
Background: Metabolic syndrome has been previously associated with increased endometrial cancer risk, but the relationship with metabolic syndrome and endometrial cancer survival remains unclear.
\end{abstract}

Objectives: To determine the associations between metabolic syndrome using the harmonized criteria with disease-free survival, overall survival, endometrial cancer-specific survival and time to recurrence among endometrial cancer survivors. Second, to determine the association between the number of metabolic syndrome components as well as each individual metabolic syndrome component with these prognostic outcomes.

Methods: A prospective cohort of 540 endometrial cancer survivors diagnosed between 2002 and 2006 participated in the Alberta Endometrial Cancer Cohort and were followed until death or March 20, 2019. Baseline in-person interviews, direct anthropometric measurements and fasting blood samples were used to assess metabolic syndrome. Recurrence and survival data were obtained via medical chart abstraction and vital status updates.

Results: Compared to endometrial cancer survivors without metabolic syndrome, survivors with metabolic syndrome had worse overall survival when assessed with the harmonized criteria. Of the individual metabolic syndrome components, only waist circumference was associated with recurrence and survival outcomes. Lifetime recreational physical activity prior to diagnosis was observed to modify the associations between metabolic syndrome and its components with overall survival and disease-free survival.

Conclusion: The metabolic syndrome, especially waist circumference, was associated with worse overall and disease-free survival among endometrial cancer survivors. Future research should aim to confirm these results and improve our understanding of the role lifestyle factors 
such as physical activity have in the association between metabolic syndrome and endometrial cancer survivors' prognosis. 


\section{Preface}

Chapter Four includes the main manuscript from this thesis which addresses the association between metabolic syndrome and its components defined by harmonized criteria with disease-free survival, overall survival, endometrial cancer-specific survival and recurrence among endometrial cancer survivors from the Alberta Endometrial Cancer Cohort Study.

For this manuscript Renee Kokts-Porietis led the formal analysis and interpretation of data, as well as drafting, writing, reviewing and editing of the manuscript. All authors were involved in conceptualization and provided critical review of this manuscript. Kerry Courneya, Linda Cook and Christine Friedenreich were involved in the funding acquisition, methodology, investigation and project administration of the larger project. Finally, Jessica McNeil, Gregg Nelson and Christine Friedenreich provided supervision and guidance to Renee Kokts-Porietis at each stage of the project.

The citation for the manuscript is:

Kokts-Porietis RL, McNeil J, Nelson GS, Courneya KS, Cook LS, Friedenreich CM. Prospective Cohort Study of Metabolic Syndrome and Endometrial Cancer Survival. Gynecol Oncol. 2020. https://doi.org/10.1016/j.ygyno.2020.06.488 


\section{Acknowledgements}

First and foremost, I would like to sincerely thank my supervisor, Dr. Christine Friedenreich for her incredible support during my thesis and beyond. I am truly grateful of the dedication and enthusiasm she has brought to this entire journey. Her mentorship and leadership have had a tremendous impact not only on my professional development but also my personal growth. Christine, thank you for your unwavering confidence in me to succeed, it has made all the difference. I would also like to acknowledge and thank each of my supervisory committee members for their support and guidance throughout this thesis: Dr. Gregg Nelson for his wholehearted engagement and insights in this project, Dr. Linda Cook for always providing incredibly thoughtful feedback that has continuously encouraged me to strive for more, and Dr. Kerry Courneya for his wonderful support and academic experience. I am tremendously grateful to you all.

I also must express my sincere appreciations to the individuals in the Department of Cancer Epidemiology and Prevention Research who have supported, encouraged and ensured I was well-caffeinated throughout this experience. I would especially like to extend my gratitude to Dr. Jessica McNeil - I could not have asked for better mentor. Her support over the last few years and the role she has played in my growth and success cannot be overstated. I would also like to thank Chelsea Stone, Rebecca Urbat and Emily Heer for their continued friendship and perspectives throughout this Master's degree.

On a personal note, I would like to offer a special thanks to my family and friends for the care and support they have provided. In particular, I would like to express my gratitude to Adrien Gosselin who has given me his unconditional support and encouragement during this degree. 
Finally, I would like to acknowledge and to extend my thanks to the endometrial cancer survivors who participated in the Alberta Endometrial Cancer Cohort, without whom this research would not be possible. I would also like to recognize the generous support from the Canadian Institutes for Health Research and the University of Calgary for the graduate scholarships that I was awarded. 


\section{Table of Contents}

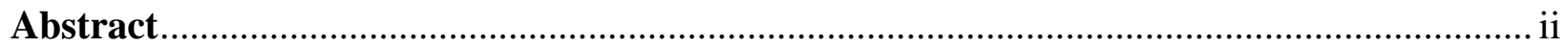

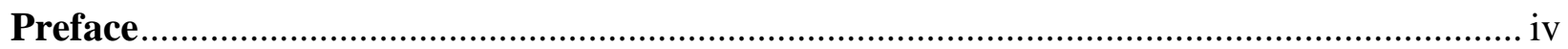

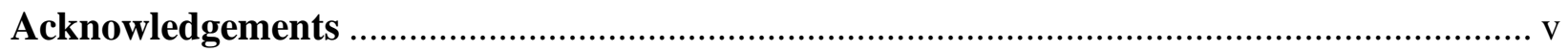

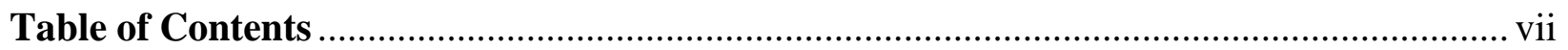

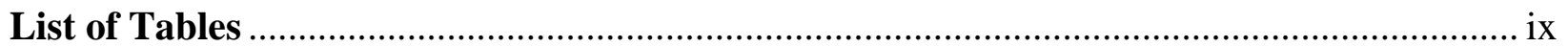

List of Illustrations, Figures \& Graphics.....................................................................

List of Symbols, Abbreviations \& Nomenclature ............................................................ xi

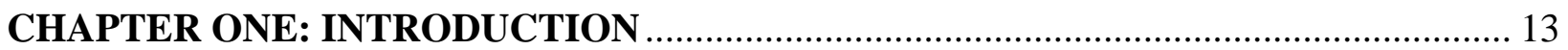

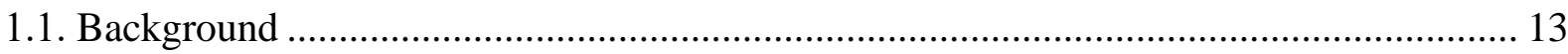

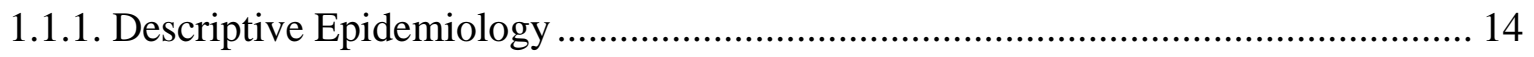

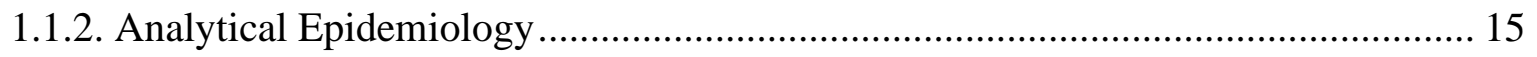

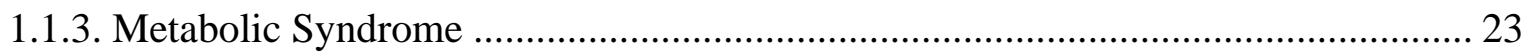

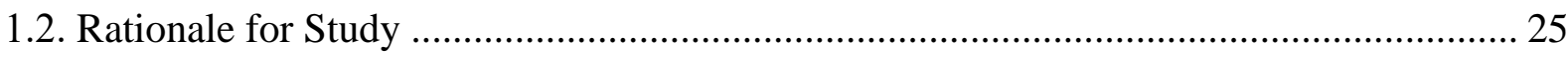

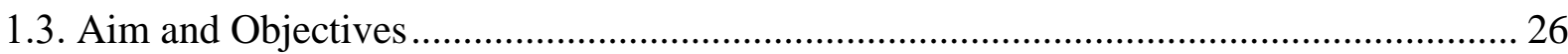

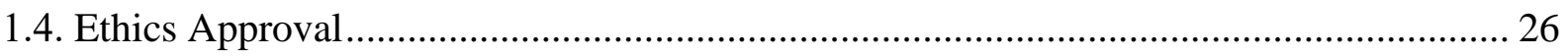

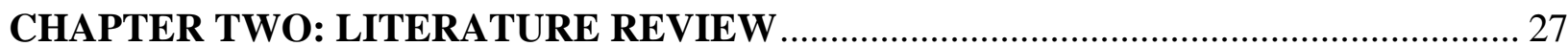

2.1. Metabolic Syndrome and Endometrial Cancer Survival........................................... 27

2.2. Metabolic Syndrome Components and Endometrial Cancer Survival .......................... 39

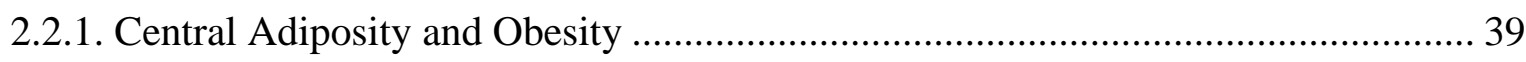

2.2.2. Hyperglycemia and Diabetes ....................................................................... 42

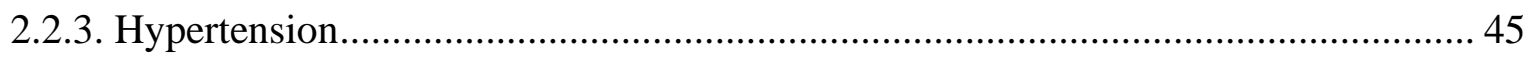

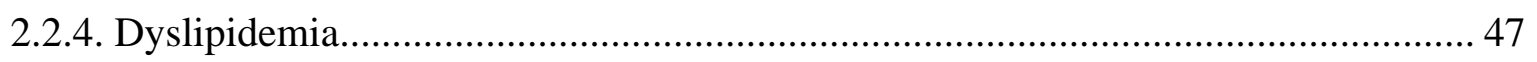

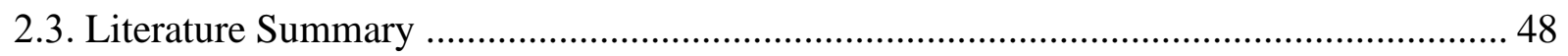

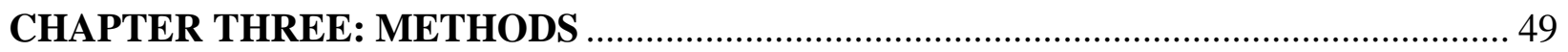

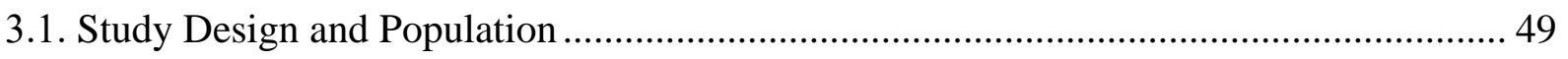

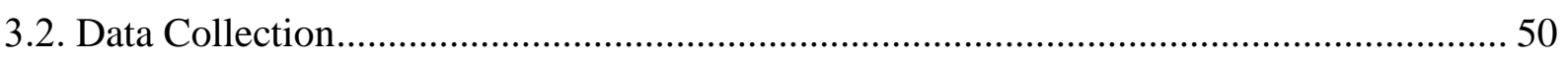

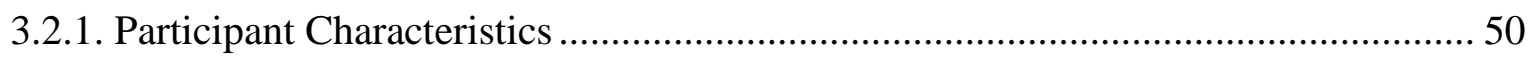

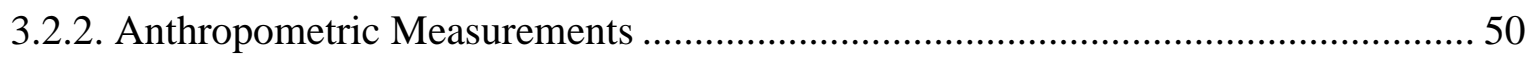

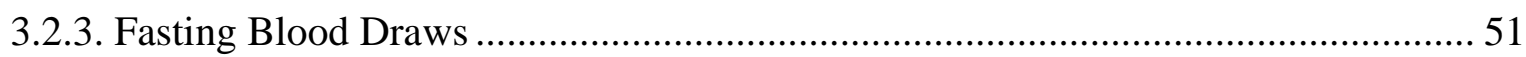

3.2.4. Medical Chart Abstraction and Vital Status Assessment ...................................... 52

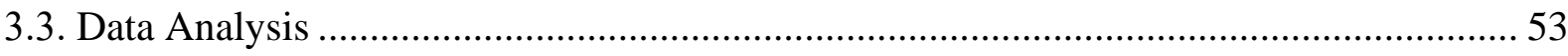

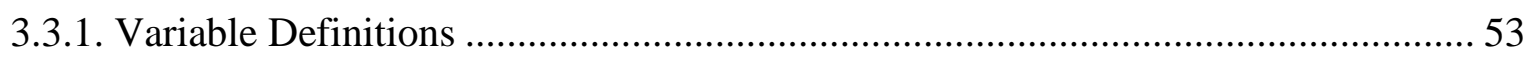

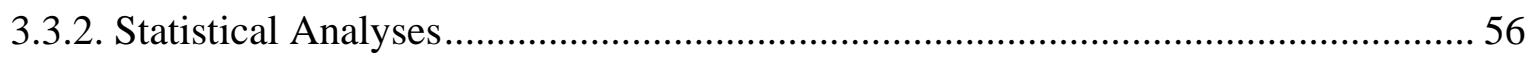


4.1. Prospective Cohort Study of Metabolic Syndrome and Endometrial Cancer Survival .... 60

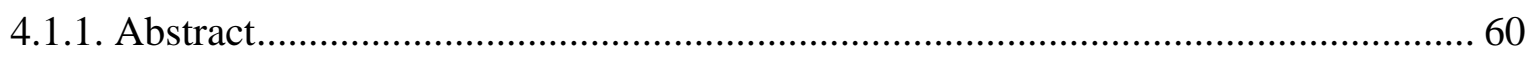

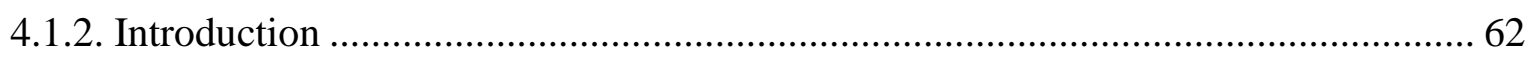

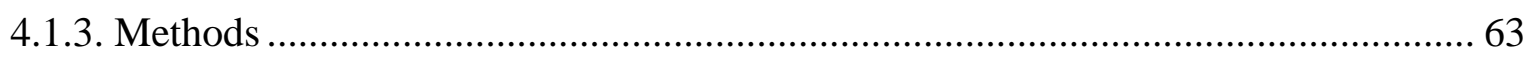

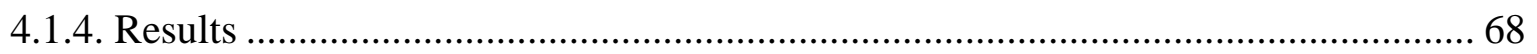

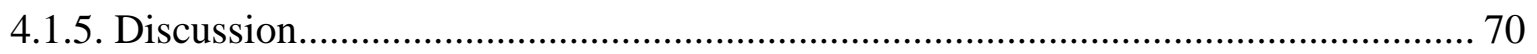

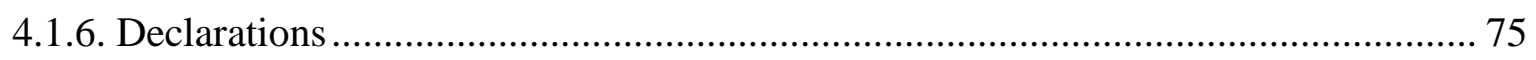

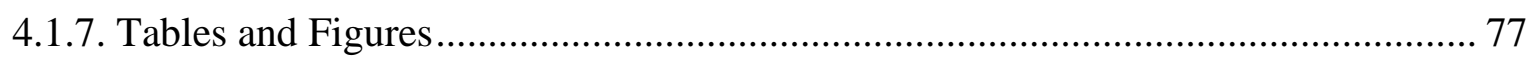

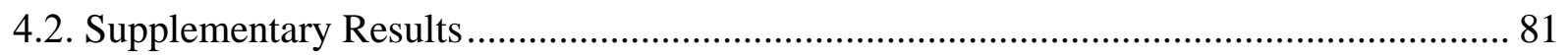

4.2.1. Medication Alternative Metabolic Syndrome Analysis .......................................... 81

4.2.2. Five- and Ten-Year Restricted Analyses............................................................ 82

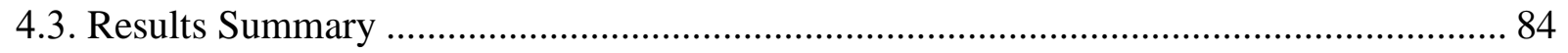

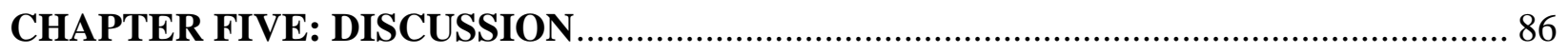

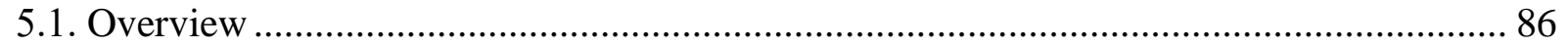

5.2. Previous Research on Metabolic Syndrome and Endometrial Cancer Survival ............... 86

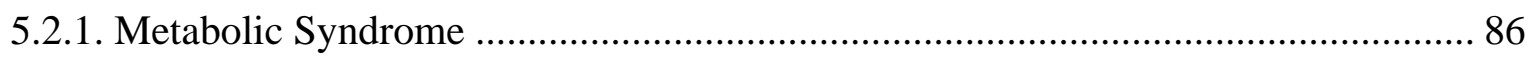

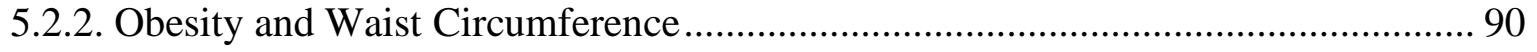

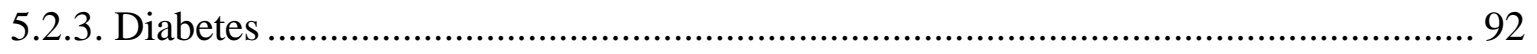

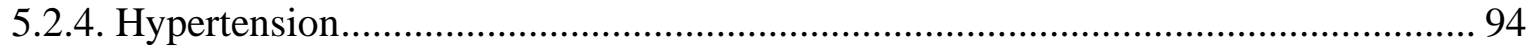

5.2.5. Triglycerides and HDL Cholesterol ................................................................... 96

5.2.6. Physical Activity Modification............................................................................. 97

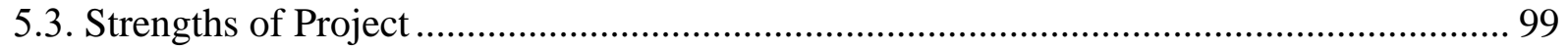

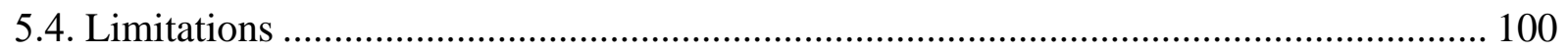

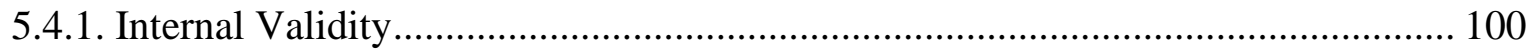

5.4.2. External Validity ........................................................................................ 105

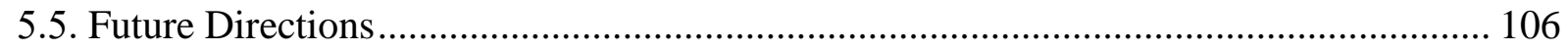

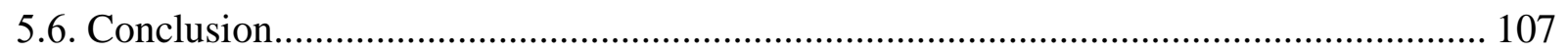

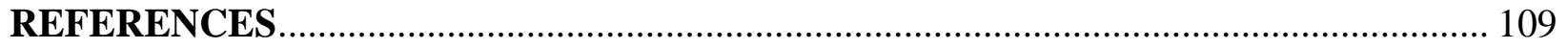

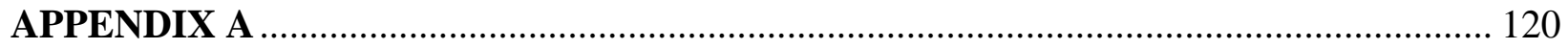

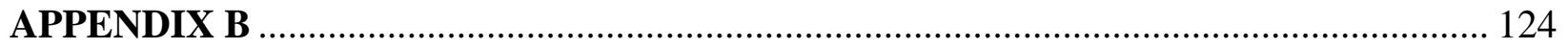




\section{List of Tables}

Table 1. Metabolic Syndrome Definitions

Table 2. Literature Summary of Metabolic Syndrome and Multiple Metabolic Syndrome Components with Endometrial Cancer Recurrence and Survival Outcomes 29

Table 3. Participant Characteristics of the Alberta Endometrial Cancer Cohort by Vital Status, Alberta, Canada $(\mathrm{N}=540)$ 77

Table 4. Disease-Free Survival, Overall Survival, Endometrial Cancer-Specific Survival and Recurrence Outcomes for Endometrial Cancer Survivors in the Alberta Endometrial Cancer Cohort, Alberta, Canada $(\mathrm{N}=520)$ 79

Table 5. Disease-Free Survival, Overall Survival, Endometrial Cancer-Specific Survival and Recurrence Outcomes for Endometrial Cancer Survivors with the Medication Alternate Metabolic Syndrome Definition in the Alberta Endometrial Cancer Cohort, Alberta, Canada $(\mathrm{N}=521)$ 82

Table 6. Five- and Ten-Year Disease-Free Survival, Overall Survival and Endometrial CancerSpecific Survival Outcomes for Endometrial Cancer Survivors by Number of Metabolic Syndrome Components in the Alberta Endometrial Cancer Cohort, Alberta, Canada $(\mathrm{N}=520) . .83$ 


\section{List of Illustrations, Figures \& Graphics}

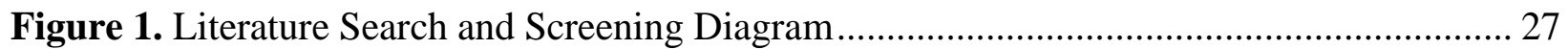

Figure 2. Participant Flowchart for the Alberta Endometrial Cancer Cohort, Alberta, Canada .. 80 


\section{List of Symbols, Abbreviations \& Nomenclature}

\begin{tabular}{ll} 
Symbols \& Abbreviations & Definition \\
$>$ & Greater than \\
$\geq$ & Less than \\
$\leq$ & Greater than or equal to \\
\pm & Less than or equal to \\
BMI & Plus or minus \\
CI & Body Mass Index \\
DBP & Confidence Interval \\
EC & Diastolic Blood Pressure \\
ESMO & Endometrial Cancer \\
FIGO & European Society of Medical Oncology \\
FU & International Federation of Gynecology and Obstetrics \\
HR & Follow-up \\
HDL & Hazard Ratio \\
HUNT & High-Density Lipoprotein \\
IARC & Health Surveys in North-Trondelag study \\
IDF & International Agency for Research on Cancer \\
IGF & International Diabetes Federation \\
LDL & Insulin-Like Growth Factor \\
Me-Can & Low Density lipoprotein \\
\hline
\end{tabular}


MET

NCEP-ATP III

NIH-AARP

NOS

NR

OR

PCOS

RR

SBP

SEER

SMR

SUCCEED

TNM

WCRF-CUP
Metabolic-Equivalent Task

National Cholesterol Education Program Adult Treatment

Panel III

National Institutes of Health- American Association of

Retired Persons

Not Otherwise Specified

Not Reported

Odds Ratio

Polycystic Ovary Syndrome

Risk Ratio

Systolic Blood Pressure

Surveillance, Epidemiology, and End Results Database

Standardized Mortality Rate

Survivors of Uterine CanCer Empowered by Exercise and

Healthy Diet

Tumour, Lymph Node, Metastasis stage

World Cancer Research Fund-Continuous Update Project 


\section{CHAPTER ONE: INTRODUCTION}

\subsection{Background}

Endometrial cancer is the sixth most commonly diagnosed cancer among women worldwide. ${ }^{1}$ Endometrial cancer accounts for $75-80 \%$ of all uterine cancers and occurs most often in women after age sixty. ${ }^{2,3}$ Based primarily on histology, endometrial cancer has been classified into two broad types: Types I and II. Type I endometrial cancers include low International Federation of Gynecology and Obstetrics (FIGO)-grade endometrioid adenocarcinomas and make up $80-90 \%$ of all endometrial cancer cases. ${ }^{3}$ These tumours typically arise following endometrial hyperplasia in the presence of hormonal imbalances, specifically high levels of unopposed estrogens. ${ }^{4}$ Lower FIGO grades (grades 1 and 2) generally have better prognosis which likely contributes to the higher survival rates observed among women living with Type I endometrial cancers. ${ }^{3}$ Type II endometrial cancers are less common and include nonendometrioid and high grade (FIGO grade 3 ) endometrioid tumours. ${ }^{3}$ These tumours are thought to develop in an atrophic environment and are considered more aggressive with higher rates of recurrence and mortality. ${ }^{3,4}$

Specific features of endometrial cancer diagnosis including histology, grade and age at diagnosis are associated with endometrial cancer survival. Relative to women with low-grade endometrioid cancer, women with high-grade endometrioid (hazard ratio $(\mathrm{HR})=6.69,95 \%$ confidence interval $(\mathrm{CI})=4.03-11.10)$, serous, clear cell, mixed endometrioid and serous/clear cell $(\mathrm{HR}=4.00,95 \% \mathrm{CI}=2.40-6.65)$, and carcinosarcoma $(\mathrm{HR}=10.20,95 \% \mathrm{CI}=5.58-18.60)$ have reduced endometrial cancer-specific survival. ${ }^{5}$ Moreover, high grade endometrial cancer $\left(\mathrm{HR}_{\text {stage }}\right.$ 4 vs $1=9.69,95 \% \mathrm{CI}=5.22-18.00)$ has been associated with decreased cancer-specific survival. ${ }^{5}$ 
Increasing age at diagnosis has also been related to reduced endometrial cancer-specific survival $\left(\mathrm{HR}_{\text {per year }}=1.06,95 \% \mathrm{CI}=1.04-1.09\right) .^{5}$

Endometrial cancer survivors have a greater prevalence of obesity, diabetes/elevated blood glucose levels (i.e. hyperglycemia), high blood pressure (i.e. hypertension) and high cholesterol (i.e. hypercholesterolemia) than the general population. ${ }^{6}$ Women living with obesity or diabetes are known to have an increased risk of developing endometrial cancer and nearly $20 \%$ of all Canadian endometrial cancer cases identified in 2015 were attributable to excess body fatness. ${ }^{7}$ In comparison genetic mutations such as Lynch and Cowden syndromes only accounted for approximately five percent of endometrial cancer cases. ${ }^{3}$ There is currently insufficient evidence to suggest that survival rates for women with Lynch syndrome-related endometrial cancer are different from survivors with sporadic endometrial cancer. ${ }^{8}$ However, endometrial cancer survivors living with obesity, diabetes or cardiovascular disease may experience reduced survival. ${ }^{5}$ The relationship between these cardiometabolic risk factors and endometrial cancer survival has been investigated to varying degrees and will be described in Chapter Two. This thesis will focus on the associations between the clustering of cardiometabolic risk factors, namely the metabolic syndrome, and survival outcomes in women living with endometrial cancer.

\subsubsection{Descriptive Epidemiology}

In 2018, the International Agency for Research on Cancer (IARC) estimated that 382,069 new cases of endometrial cancer and 89,929 endometrial cancer-specific deaths occurred globally ${ }^{1}$ with the highest rates occurring in North America and Central and Eastern Europe. ${ }^{1,9}$ Approximately 7,400 new diagnoses and 1,300 endometrial cancer-specific deaths are estimated 
to occur in 2020, and the lifetime probability of dying from endometrial cancer in Canada is currently one in $147 .^{10,11}$ In recent years, uterine cancer mortality rates increased two percent annually in Canada and the USA, making endometrial cancer one of the few cancer types with both increasing incidence and mortality rates. ${ }^{11,12}$

The median age for peak uterine cancer incidence has decreased between 1992 and 2010 from 66 years to 62 years of age among Canadian women, ${ }^{2}$ whereas the median age of death has increased during this time with the highest mortality rates occurring among survivors over the age of 85 years. ${ }^{2}$ Within the American context, White women experience greater incidence of endometrial cancer and are typically diagnosed at a younger age than Black women. ${ }^{4,13}$ In contrast, Black women experience nearly double the rate of endometrial cancer-specific mortality. $^{13}$

\subsubsection{Analytical Epidemiology}

The World Cancer Research Fund Continuous Update Project (WCRF-CUP) investigates diet, nutrition and physical activity factors related to cancer prevention and survival. ${ }^{9}$ While several risk factors have been investigated in the etiology of endometrial cancer, only greater body fatness including abdominal fatness and adult weight gain, has been deemed a convincing cause of endometrial cancer. ${ }^{9}$ The WCRF has reported that there is probable evidence that physical activity, coffee consumption, adult attained height as a proxy for developmental factors during childhood and glycemic load are related to endometrial cancer risk, while there is limited evidence that suggests sedentary behavior is related to the risk of endometrial cancer. ${ }^{9}$

Reproductive life events, medication use and chronic conditions have also been associated with endometrial cancer incidence and survival to varying degrees. ${ }^{9}$ 


\subsubsection{Lifestyle Factors}

Currently, Body Mass Index $\left(\mathrm{BMI}, \mathrm{kg} / \mathrm{m}^{2}\right)$ is the most commonly used method for assessing body fatness and classify individuals as overweight $\left(25-29.9 \mathrm{~kg} / \mathrm{m}^{2}\right)$ or obese (> 30 $\left.\mathrm{kg} / \mathrm{m}^{2}\right) .{ }^{14}$ However, it has been suggested that central adiposity (waist circumference) may be more relevant for morbidity and mortality outcomes. ${ }^{14}$ Obesity is considered to be a convincing cause of endometrial cancer as many pathological features of body fatness such as metabolic disruptions, hormonal and growth factor imbalances, and chronic inflammation prompt tumour growth. ${ }^{14-16}$ Individuals living with cancer and obesity also experience reduced responses to standard treatments, higher rates of metastatic disease and generally poorer prognoses than normal weight individuals. ${ }^{16}$ The link between adipose tissue and female sex hormones is thought to drive the association between obesity and endometrial cancer pathogenesis. ${ }^{14}$ Adipose tissue can convert androgens and androgenic precursors into the female sex steroid estrogen. In individuals with obesity, excess adipose tissue results in greater conversion rates and thus higher estrogen levels. ${ }^{14}$ Unopposed estrogen in the endometrium stimulates cell proliferation and inhibition of apoptosis, which then promotes tumourigenesis. ${ }^{14}$ This mechanism of endometrial cancer pathogenesis is known as the unopposed estrogen hypothesis. In a systematic review and meta-analysis, Aune et al. ${ }^{15}$ reported a non-linear increase in endometrial cancer risk for every five-unit increase in BMI, with greater risk being observed in the overweight and obese BMI ranges. Moreover, for each ten $\mathrm{cm}$ increase in waist circumference the risk of endometrial cancer increased $1.27(95 \% \mathrm{CI}=1.17-1.39)$ times. ${ }^{15}$ Obesity may also decrease endometrial cancerspecific survival, as well as overall survival in women living with endometrial cancer. ${ }^{14}$ 
Endometrial cancer survivors with obesity have a statistically significant decrease in overall

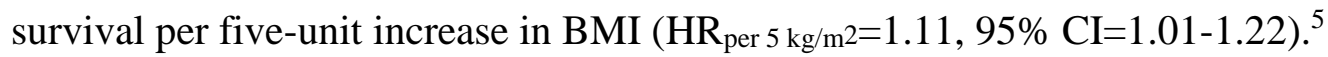

Women with high compared to low physical activity levels have a reduced risk of endometrial cancer (risk ratio $(\mathrm{RR})=0.80,95 \% \mathrm{CI}=0.75-0.85),{ }^{17}$ whereas high rather than low amounts of sedentary behaviour may increase the risk of endometrial cancer by approximately $34 \% .^{18} \mathrm{~A}$ recent meta-analysis reported that post-diagnosis physical activity was associated with improved overall survival in a combined estimate of all female reproductive cancers ( $\mathrm{HR}=0.66$, 95\% CI=0.49-0.88).$^{19}$ No relationship was observed in this meta-analysis for pre-diagnosis physical activity or cancer-specific survival for female reproductive cancers survivors. ${ }^{19}$

There is limited evidence whether or not dietary patterns or specific foods impact endometrial cancer risk. ${ }^{9}$ The WCRF-CUP reported a $15 \%$ increased risk in endometrial cancer per 50 units of glycemic load increase per day $\left(R_{\text {per }} 50\right.$ glycemic load units/day $=1.15,95 \% \mathrm{CI}=1.06-$ 1.25). ${ }^{9}$ It has also been suggested that prolonged high glycemic load diets can lead to hyperinsulinemia and result in greater bioavailability of insulin-like growth factor-1 (IGF), ${ }^{20}$ which can directly promote endometrial cancer cell division and growth. ${ }^{20}$ Currently there is no available evidence on the relationship between glycemic load and endometrial cancer recurrence or survival, and a null association has been reported for the relationship between glycemic load and overall survival among women in the general population $(\mathrm{RR}=1.06,95 \% \mathrm{CI}=0.90-1.25) .{ }^{21}$

There is some evidence that greater adult attained height is associated with increased endometrial cancer risk. The WCRF-CUP reported that for every five $\mathrm{cm}$ increase in adult attained height the risk of endometrial cancer increased seven percent $(95 \% \mathrm{CI}=3-11 \%)$ in a meta-analyses of ten studies. ${ }^{9}$ Adult height is a marker of developmental factors during childhood such as the rate of growth, the number of cell divisions, age of sexual maturity and is a 
proxy for nutritional exposures which affect several hormonal and metabolic mechanisms. ${ }^{9}$ Specifically, taller women may have been exposed to greater insulin levels, pituitary-derived growth hormone and IGFs. ${ }^{9}$

Greater coffee consumption, including decaffeinated coffee, may reduce the risk of endometrial cancer $(\mathrm{RR}=0.74,95 \% \mathrm{CI}=0.68-0.81) .{ }^{9,22}$ While the specific biological mechanism remains unknown, the link between coffee consumption and reduced risk of endometrial cancer may be related to lower circulating levels of estrogen or insulin as well as higher insulin sensitivity. ${ }^{9}$ Coffee has also been associated with reduced obesity, inflammation and bioavailability of estrogens, which may partiality explain its protective association with endometrial cancer. ${ }^{9,22}$ The association between coffee consumption and recurrence or survival outcomes among endometrial cancer survivors has not been assessed in the literature.

Although not included in the WCRF-CUP because it was outside the scope of that report, cigarette smoking has been found to reduce endometrial cancer risk. ${ }^{23}$ Results from a metaanalysis suggest that postmenopausal women who currently smoke have between a 30-40\% lower risk of endometrial cancer compared to never smokers. ${ }^{23}$ While this association was slightly attenuated among ever smokers, a 20-30\% reduction in risk of endometrial cancer was observed for ever compared to never smokers. ${ }^{23}$ These findings may be related to lower BMI commonly observed among individuals who smoke and thus reduced endogenous estrogen levels, as well as higher progesterone levels reported among smokers. ${ }^{4}$ However, it is important to note that while current smokers may be at a reduced risk of endometrial cancer, they experience half the overall survival as never smokers. ${ }^{24}$ Additionally, endometrial cancer survivors who smoke have reduced endometrial cancer-specific survival (HR=1.38, 95\% 
$\mathrm{CI}=1.09-1.75)$ and overall survival $(\mathrm{HR}=1.41,95 \% \mathrm{CI}=1.20-1.66)$ compared to survivors who are non-smokers. $^{25}$

\subsubsection{Events of Reproductive Life}

Reproductive factors such as age at menarche, menopausal age, parity and anovulation have been previously associated with the risk of endometrial cancer. ${ }^{4}$ These reproductive events are closely linked to endogenous hormonal levels, namely estrogens which are central to endometrial cancer etiology. ${ }^{4}$ As mentioned above, the unopposed estrogen hypothesis suggests that high levels of estrogen in the absence of sufficient progesterone increases the risk of endometrial cancer by promoting endometrial proliferation that can lead to malignant transformations. ${ }^{4,26}$ Women living with polycystic ovary syndrome (PCOS) experience chronic anovulation which results in high unopposed estrogen levels. ${ }^{4}$ Subsequently, women with PCOS are at an elevated risk of endometrial cancer (odds ratio $(\mathrm{OR})=2.79,95 \% \mathrm{CI}=1.31-5.95) .{ }^{27} \mathrm{To}$ date, there are no known studies that have assessed the association between PCOS and survival among endometrial cancer survivors. Although there is some evidence that women living with PCOS in the general population may have reduced overall survival, these association did not reach statistical significance $(\mathrm{OR}=1.21,95 \% \mathrm{CI}=0.91-1.60) .{ }^{28}$ Older age at menarche may be protective for endometrial cancer incidence later in life $\left(\mathrm{RR}_{\text {older vs younger }}=0.68,95 \% \mathrm{CI}=0.58\right.$ $0.81),{ }^{29}$ while later age of menopause onset has been associated with an increased risk of endometrial cancer. ${ }^{26}$ Specifically among studies from North America, women with the oldest age at menopause onset had $1.60(95 \% \mathrm{CI}=1.37-1.86)$ times the risk of endometrial cancer compared to women with the youngest age at menopause onset. ${ }^{26}$ No studies have assessed the relationship between age of menopause and subsequent endometrial cancer recurrence or 
survival. A meta-analysis of epidemiologic studies reported an overall protective association between parity and endometrial cancer risk $\left(\mathrm{RR}_{\text {parity vs nulliparous }}=0.69,95 \% \mathrm{CI}=0.65-0.74\right) .{ }^{30}$ Parity may also improve overall survival $\left(\mathrm{HR}_{\text {parity vs nulliparous }}=0.69,95 \% \mathrm{CI}=0.58-0.82\right) .{ }^{31} \mathrm{It}$ is hypothesized that the relationship between parity and endometrial cancer is related to the hormonal changes that occur during pregnancy. ${ }^{32}$

\subsubsection{Exogenous Hormones}

The use of pre- and post-menopausal estrogen only hormones has been consistently shown to increase the risk of endometrial cancer, while there have been inconsistent findings associated with combined use of estrogen and progestin. ${ }^{33}$ Several reasons exist for the lack of direct associations between combined hormone therapy use and endometrial cancer risk. The most important being the protective effect of progestins on endometrial cancer risk, as well as the wide range of regimens of combined estrogen and progestin that are commonly utilized. ${ }^{4}$ Endometrial cancer recurrence was not associated with estrogen only hormone therapy at the time of diagnosis $(\mathrm{OR}=0.78,95 \% \mathrm{CI}=0.38-1.57)$ and a protective association was observed in a meta-analysis of observational studies with combined estrogen and progestin use (OR=0.23, 95\% $\mathrm{CI}=0.08-0.66) .{ }^{34}$

There has been limited research on hormonal therapy use and survival among endometrial cancer survivors. Most recently, Felix et al. ${ }^{35}$ observed that pre-diagnosis use of estrogen and progestin therapy was associated with better ten-year overall survival $(\mathrm{HR}=0.65$, 95\% CI=0.43-0.99) and endometrial cancer-specific survival $(\mathrm{HR}=0.51,95 \% \mathrm{CI}=0.26-0.98)$ within the National Institutes of Health-American Association of Retired Persons cohort (NIH- 
AARP). Since these estimates were based on a small number of deaths (29 and 11 deaths, respectively), further research is warranted to confirm these results. ${ }^{35}$

\subsubsection{Chronic Conditions}

Women living with diabetes have nearly double $(\mathrm{RR}=1.72,95 \% \mathrm{CI}=1.48-2.01)$ the risk

of endometrial cancer. ${ }^{36}$ Increased fasting glucose levels and insulin resistance are hallmarks of diabetes. ${ }^{37}$ Bjorge et al. ${ }^{38}$ previously reported that women in the highest quintile of fasting glucose levels (mmol/L) compared to the lowest had 1.57 (95\% CI=1.27-1.95) times the risk of endometrial cancer. Similarly, Arthur et al. ${ }^{39}$ observed double $(\mathrm{RR}=1.99,95 \% \mathrm{CI}=1.36-2.49)$ the risk of endometrial cancer for women with fasting glucose levels $\geq 100 \mathrm{mg} / \mathrm{dL}$ compared to < $100 \mathrm{mg} / \mathrm{dL}$. Elevated glucose levels can provide tumours with the energy needed to promote cancer growth, cancer progression and ultimately lead to decreased survival. ${ }^{14}$ While diabetes has been found to decrease both cancer-specific survival and overall survival in women with endometrial cancer, the body of literature on this topic remains inconclusive.${ }^{37}$ In a populationbased study of endometrial cancer survivors in the Netherlands, five-year overall survival rates were significantly lower for endometrial cancer survivors with diabetes (68\%) compared to survivors without diabetes (84\%). ${ }^{40}$ Endometrial cancer survivors with diabetes have been reported to experience a 1.6-fold decrease in overall survival compared to survivors without diabetes $\left(\mathrm{HR}=1.58,95 \% \mathrm{CI}=1.07-2.33,{ }^{41} \mathrm{HR}=1.7,95 \% \mathrm{CI}=1.1-2.5\right) .{ }^{42}$ More recently, Nagel et al. ${ }^{5}$ reported that endometrial cancer survivors living with diabetes also had poor endometrial cancer-specific survival $(\mathrm{HR}=2.09,95 \% \mathrm{CI}=1.31-3.35)$.

Several cardiovascular disease risk factors are also closely associated with endometrial cancer risk. Participants of the Women's Health Initiative cohort study with low high-density 
lipoprotein (HDL) cholesterol ( $<50 \mathrm{mg} / \mathrm{dL}$ ) or elevated triglycerides levels $(\geq 150 \mathrm{mg} / \mathrm{dL}$ ) had $1.65(95 \% \mathrm{CI}=1.22-2.23)$ and $1.34(95 \% \mathrm{CI}=0.97-1.85)$ times the risk of endometrial cancer compared to participants with normal lipid level, respectively. ${ }^{39}$ Bjorge et al. ${ }^{38}$ also noted that in contrast to women with the lowest quintile of triglycerides, those with the highest levels had a greater risk of endometrial cancer $(\mathrm{RR}=1.61,95 \% \mathrm{CI}=1.29-2.02$, $\mathrm{p}$-trend $<0.01)$. Moreover, Kitson et al. ${ }^{6}$ reported that significantly more ( $88.7 \%$ versus $\left.54.3 \%\right)$ endometrial cancer survivors had undiagnosed or undertreated cardiovascular disease risk factors compared to women in the overall UK population. The authors also noted that despite endometrial cancer survivors already taking significantly more antihypertensive medication compared to the general population, nearly $50 \%$ of survivors were still classified as hypertensive. ${ }^{6}$ Hypertension has been shown to elevate endometrial cancer risk $\left(\mathrm{HR}_{\text {per unit } z \text {-score }}=1.20,95 \% \mathrm{CI}=1.13-1.28\right) .{ }^{38}$

Five years after an endometrial cancer diagnosis, Ward et al. ${ }^{43}$ reported that the most frequent cause of death for endometrial cancer survivors becomes cardiovascular disease rather than endometrial cancer. In the Surveillance, Epidemiology, and End Results (SEER) Database, endometrial cancer survivors were reported to have greater cardiovascular disease mortality (standardized mortality rate $(\mathrm{SMR})=8.8,95 \% \mathrm{CI}=8.7-9.0)$ and all-cause mortality $(\mathrm{SMR}=15.9$, 95\% CI=15.8-16.0) than the general population. ${ }^{44}$ However, results from the Iowa Women's Health Study suggest that women living with endometrial cancer have a reduced risk of cardiovascular disease mortality ( $\mathrm{HR}=0.75,95 \% \mathrm{CI}=0.56-0.99)$, and a greater risk of all-cause mortality $(\mathrm{HR}=1.50,95 \% \mathrm{CI}=1.30-1.74)$ than the general population, after adjusting for both BMI and diabetes in the analysis. ${ }^{45}$ These findings highlight the discrepancy that currently exists in the literature regarding the contributions of obesity, diabetes and cardiovascular disease with survival outcomes among endometrial cancer survivors. 


\subsubsection{Metabolic Syndrome}

Metabolic syndrome is defined as the clustering of cardiovascular and metabolic disease risk factors including central adiposity (waist circumference $\geq 88 \mathrm{~cm}$ ), hypertension (systolic blood pressure $(\mathrm{SBP}) \geq 130 \mathrm{mmHg}$ or diastolic blood pressure $(\mathrm{DBP}) \geq 85 \mathrm{mmHg}$, hyperglycemia (fasting blood glucose levels $\geq 100 \mathrm{mg} / \mathrm{dL}$ ), and dyslipidemia (triglyceride levels $\geq 150 \mathrm{mg} / \mathrm{dL}$ and/or low HDL cholesterol levels $<50 \mathrm{mg} / \mathrm{dL}) .{ }^{46}$ The specific characteristics of the metabolic syndrome have evolved since the first formalized definition was proposed by the World Health Organization in $1998 .{ }^{46}$ A consensus statement was put forth in 2009 to harmonize the criteria of metabolic syndrome by the International Diabetes Federation (IDF), and the American Heart Association/ National Heart, Lung, and Blood Institute who had adopted the National Cholesterol Education Program Adult Treatment Panel III (NCEP-ATP III)

guidelines. ${ }^{46}$ Specific criteria of metabolic syndrome definitions are outlined in Table 1. Of note, metabolic syndrome defined with the harmonized criteria is categorized using country- and sexspecific waist circumference criteria. ${ }^{46}$ Moreover, medication use for the treatment of hypertension, dyslipidemia or hyperglycemia are considered to be alternative indicators for this metabolic syndrome criteria (i.e. medication alternative metabolic syndrome criteria). ${ }^{46}$ 
Table 1. Metabolic Syndrome Definitions

\begin{tabular}{|c|c|c|c|}
\hline Definition & IDF $2005^{43}$ & NCEP-ATP III $2005^{46}$ & Harmonized $2009^{46}$ \\
\hline & $\begin{array}{l}\text { Waist Circumference and } \\
\text { any two other components }\end{array}$ & $\begin{array}{l}\text { Any three of the } \\
\text { components }\end{array}$ & $\begin{array}{l}\text { Any three of the } \\
\text { components }\end{array}$ \\
\hline \multicolumn{4}{|l|}{ Obesity } \\
\hline Waist Circumference & $\geq 80 \mathrm{~cm}^{\mathrm{a} * *}$ & $\geq 88 \mathrm{~cm}$ & $\geq 88 \mathrm{~cm}^{\mathrm{b}}$ \\
\hline Hyperglycemia & & & \\
\hline Fasting Plasma Glucose & $\begin{array}{l}\geq 100 \mathrm{mg} / \mathrm{dL} \text { or previously } \\
\text { diagnosed Type } 2 \text { diabetes }\end{array}$ & $\geq 110 \mathrm{mg} / \mathrm{dL}$ & $\geq 100 \mathrm{mg} / \mathrm{dL}^{*}$ \\
\hline \multicolumn{4}{|l|}{ Hypertension } \\
\hline Blood Pressure & $\begin{array}{l}\mathrm{SBP} \geq 130 \mathrm{mmHg} \text { or } \\
\mathrm{DBP} \geq 85 \mathrm{mmHg} *\end{array}$ & $\begin{array}{l}\mathrm{SBP} \geq 130 \mathrm{mmHg} \text { or } \\
\mathrm{DBP} \geq 85 \mathrm{mmHg}\end{array}$ & $\begin{array}{l}\mathrm{SBP} \geq 130 \text { and/or } \\
\mathrm{DBP} \geq 85 \mathrm{mmHg} *\end{array}$ \\
\hline \multicolumn{4}{|l|}{ Dyslipidemia } \\
\hline Triglycerides & $\geq 150 \mathrm{mg} / \mathrm{dL}^{*}$ & $\geq 150 \mathrm{mg} / \mathrm{dL}$ & $\geq 150 \mathrm{mg} / \mathrm{dL}^{*}$ \\
\hline HDL Cholesterol & $<50 \mathrm{mg} / \mathrm{dL}^{*}$ & $<50 \mathrm{mg} / \mathrm{dL}$ & $<50 \mathrm{mg} / \mathrm{dL} *$ \\
\hline
\end{tabular}

*Drug treatment of condition is an alternate indicator

**If BMI is $>30 \mathrm{~kg} / \mathrm{m}^{2}$, central obesity can be assumed

aEuropids ethnic group

${ }^{\mathrm{b} C}$ Canadian Specific

Although prevalence estimates differ by the metabolic syndrome definition that is used, it is estimated that approximately one quarter of the world population has metabolic syndrome and one in five Canadians have metabolic syndrome. ${ }^{47-49}$ Moreover, endometrial cancer survivors have a greater prevalence of obesity, diabetes, non-diabetic hyperglycemia, hypertension and hypercholesterolemia compared to women in the general population. ${ }^{6}$ Additionally, most endometrial cancer survivors are expected to have more than one metabolic syndrome risk factor. 6

While metabolic syndrome is known to increase the risk of diabetes fivefold and cardiovascular disease threefold, ${ }^{49}$ it has more recently been associated with an increased risk of cancer. ${ }^{50,51}$ The estimated risk of developing endometrial cancer for women with metabolic syndrome is $1.89(95 \% \mathrm{CI}=1.34-2.67)^{52}$ to $2.20(95 \% \mathrm{CI}=1.61-3.02)^{39}$ times that experienced among women without this syndrome. Additionally, for every increase in standard deviation of a 
continuous metabolic risk score, Stocks et al. ${ }^{53}$ observed a 56\% (95\% CI=42-70\%) increased risk of endometrial cancer.

Women diagnosed with metabolic syndrome in the Third National Health and Nutrition Examination Survey experienced a 33\% (95\% CI=4-71\%) decrease in total cancer-specific survival. ${ }^{54}$ A positive relationship was identified between the number of metabolic syndrome risk factors and total cancer-specific mortality in this study (p-trend $<0.01$ ), such that compared to individuals without metabolic syndrome, those with three, four and five metabolic syndrome components had 1.25 (95\% CI=0.93-1.67), 1.33 (95\% CI=0.94-1.86), and 1.73 (95\% CI=1.122.68) times the hazard of cancer-specific mortality, respectively. ${ }^{54}$ No association between metabolic syndrome and obesity-linked (breast, colorectal, pancreatic and endometrial) cancer survival reached statistical significance in this study. ${ }^{54}$ Among endometrial cancer survivors, only four studies have assessed the association between metabolic syndrome and survival outcomes. ${ }^{38,55-57}$ Generally, metabolic syndrome has been associated with reduced endometrial cancer-specific survival ${ }^{38,55}$ and overall survival ${ }^{56,57}$ among women living with endometrial cancer.

\subsection{Rationale for Study}

The associations between endometrial cancer incidence and metabolic syndrome as well as its components have been previously identified. ${ }^{39,58}$ Despite the biological plausibility that metabolic syndrome may be associated with endometrial cancer recurrence and survival, ${ }^{14,54}$ limited information on survival outcomes currently exists. Therefore, additional research is needed to understand recurrence and survival outcomes among endometrial cancer survivors associated with metabolic syndrome. 


\subsection{Aim and Objectives}

The aim of this thesis was to examine the associations between metabolic syndrome and its components with recurrence and survival outcomes among endometrial cancer survivors. The first objective was to determine the associations between metabolic syndrome using the harmonized criteria and the medication alternative harmonized definition, ${ }^{46}$ with disease-free survival, overall survival, endometrial cancer-specific survival and time to recurrence among endometrial cancer survivors in the Alberta Endometrial Cancer Cohort. The second objective was to determine the relationships between the number of metabolic syndrome components as well as each individual metabolic syndrome component with these prognostic outcomes.

\subsection{Ethics Approval}

Ethical approval for the "Metabolic Syndrome and Lifestyle Risk Factors in Endometrial Cancer Etiology and Survival" project was obtained from the Health Research Ethics Board of Alberta- Cancer Committee (Ethics ID 17-0574). The addition of Renee Kokts-Porietis as a student co-investigator to undertake this thesis was approved on August 23, 2019. 


\section{CHAPTER TWO: LITERATURE REVIEW}

\subsection{Metabolic Syndrome and Endometrial Cancer Survival}

A comprehensive literature search was conducted to summarize the available evidence on the relations between metabolic syndrome and its components with recurrence and survival outcomes among endometrial cancer survivors. The search was completed using the MEDLINE (Ovid) and EMBASE (Ovid) databases from 1990 up to and including March 19, 2020. Three concepts were included in each database search: endometrial cancer, metabolic syndrome and its individual components, and recurrence/survival. Database subject headings (e.g. MEDLINE Subject Heading), title abstract keywords and Boolean operators were used to define and combine these concepts. The complete search strategies are provided in the Appendix A. Additional relevant literature was included at the full text stage. An overview of the article screening process is presented in Figure 1.

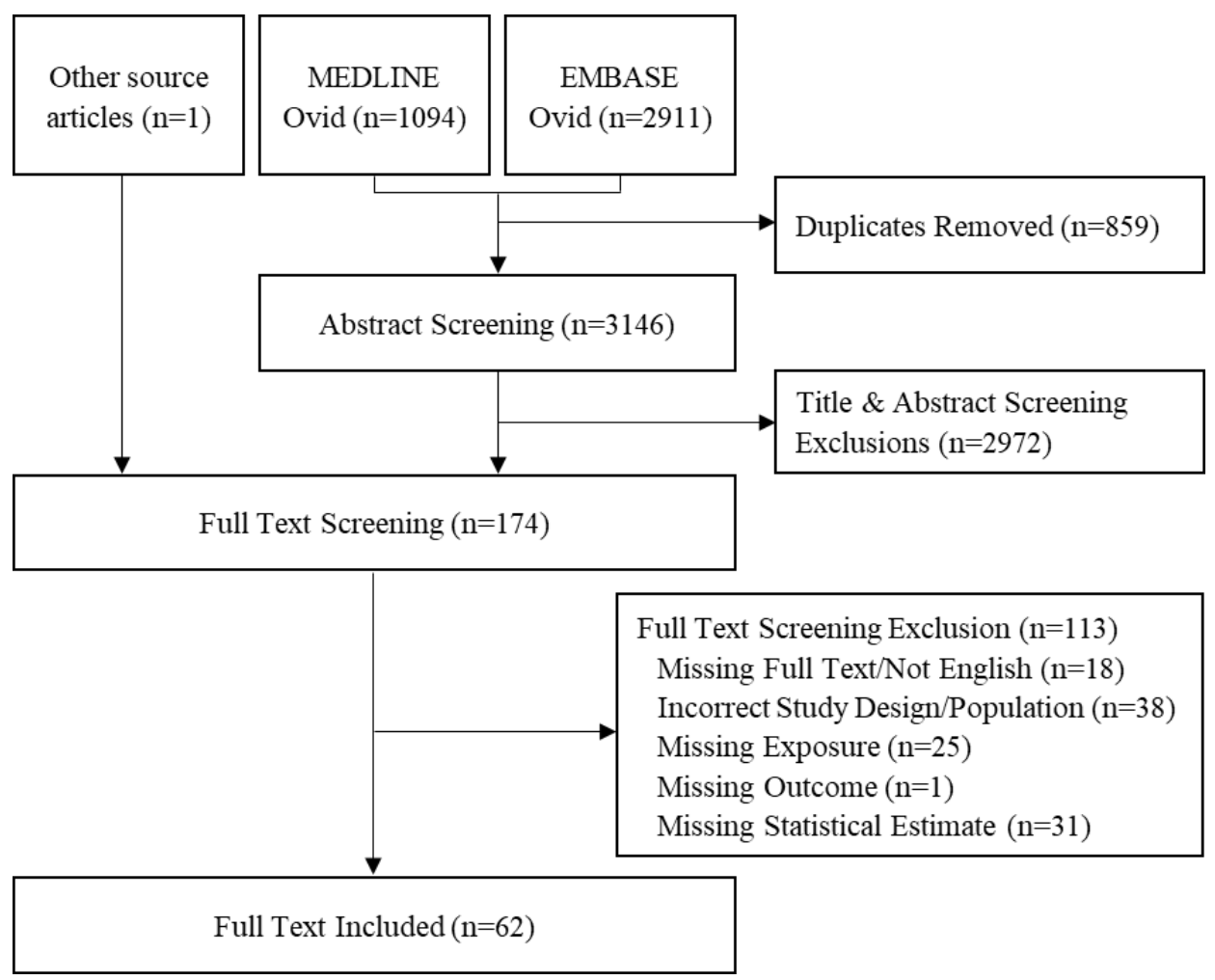

Figure 1. Literature Search and Screening Diagram 
Sixty-two full text publications reported estimates for the relationship between either metabolic syndrome or at least one of its components with overall survival, endometrial cancerspecific survival, and endometrial cancer recurrence. Forty-five $e^{5,38,41,42,55,59-98}$ of these articles provided estimates for obesity, sixteen of which were included in a systematic review and metaanalysis that summarizes the association between BMI and survival in articles published prior to June $2013 .{ }^{99}$ A total of thirty $5,38,40-42,55,70,71,75-80,84,88,90,91,94,95,100-109$ articles reported estimates for hyperglycemia, glucose levels or diabetes and recurrence or survival outcomes. There were eighteen $^{38,40,41,55,63,70,75,77,80,84,88,91,94,95,101,109-111}$ articles that included hypertension estimates and five $\mathrm{e}^{38,55,70,84,91}$ studies presented dyslipidemia estimates with the prognostic outcomes. Only four $\operatorname{articles}^{38,55-57}$ provided estimates specific to metabolic syndrome, although most of these studies used proxy metabolic syndrome criteria ${ }^{55-57}$ or study-specific metabolic syndrome definitions. ${ }^{38,56,57}$ A summary of the literature that reports estimates of the associations for metabolic syndrome or more than one metabolic syndrome component and endometrial cancer recurrence or survival outcomes is presented in Table 2. Most of this literature was conducted as retrospective analyses of large prospective cohorts or databases, ${ }^{38,40,55,70,77,79,94,95,101,109}$ twelve were retrospective cohorts derived from hospital medical records ${ }^{41,56,57,63,75,76,78,80,84,88,90,91}$ and three studies were conducted prospectively. ${ }^{5,42,71}$ Of note, while the language used to describe survival outcomes in the literature differed by study, all statistical estimates are presented in terms of recurrence or mortality events. The main findings from all identified studies are summarized in terms of survival outcomes in the following sections of this chapter. 
Table 2. Literature Summary of Metabolic Syndrome and Multiple Metabolic Syndrome Components with Endometrial Cancer Recurrence and Survival Outcomes

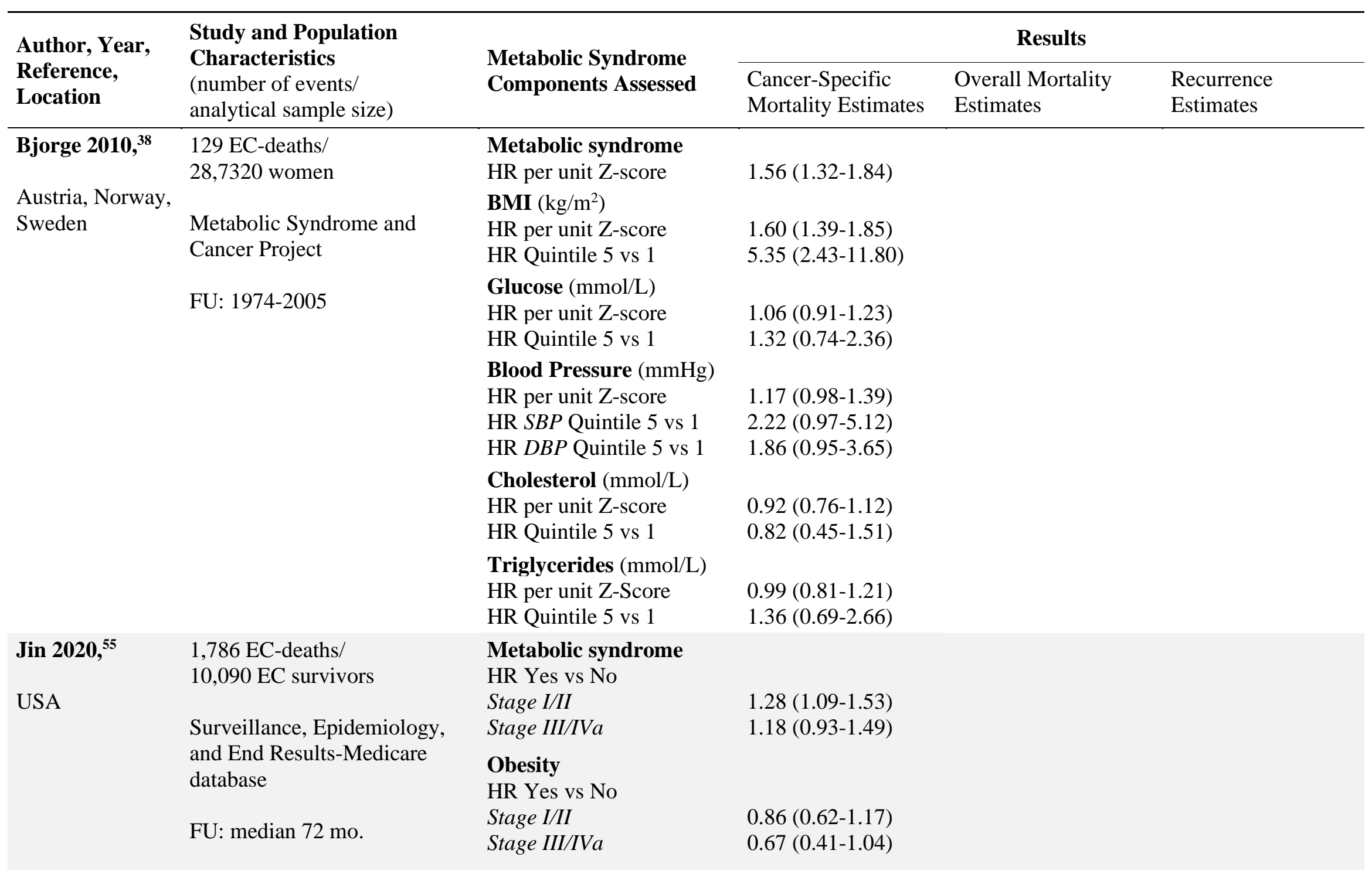




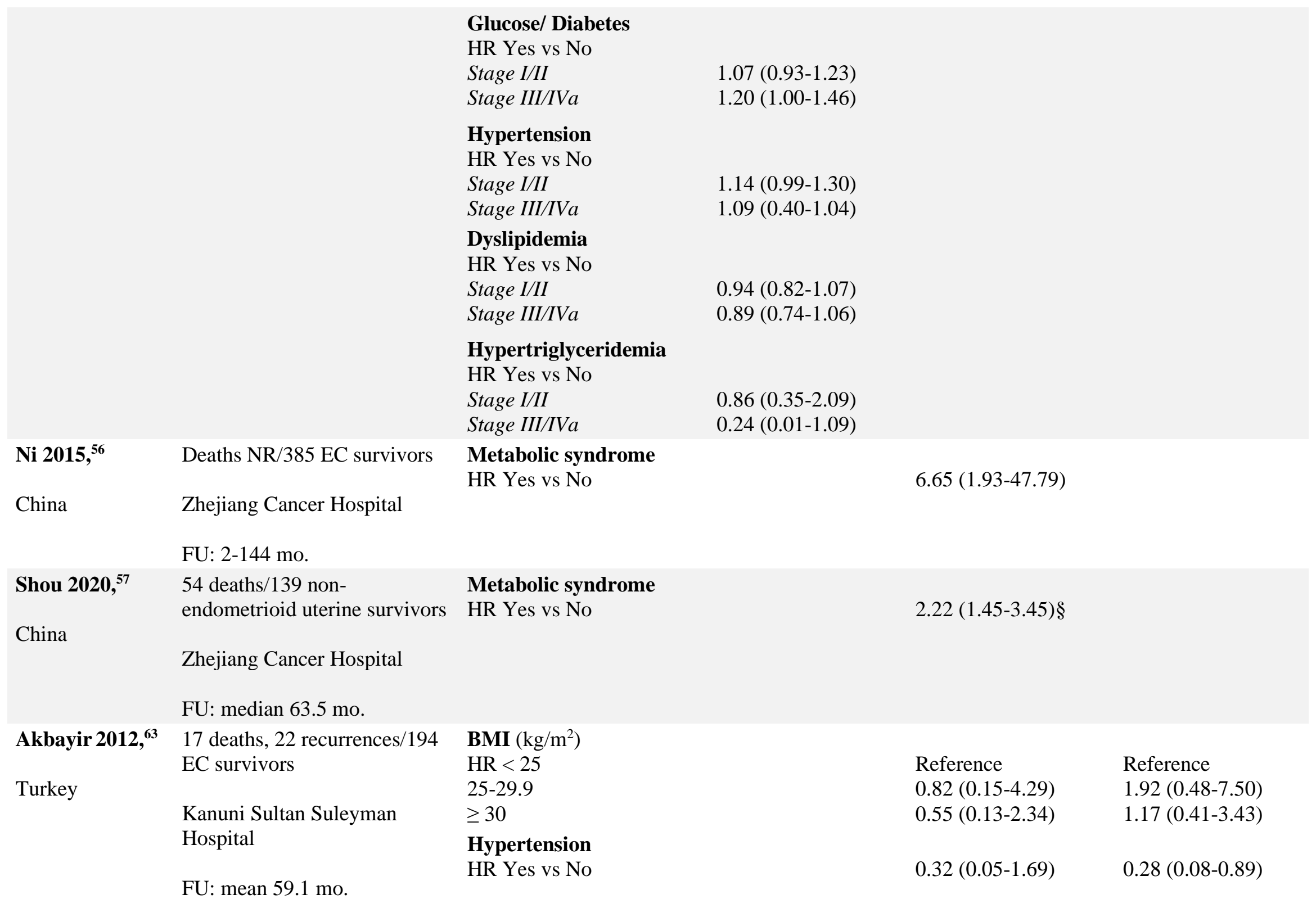




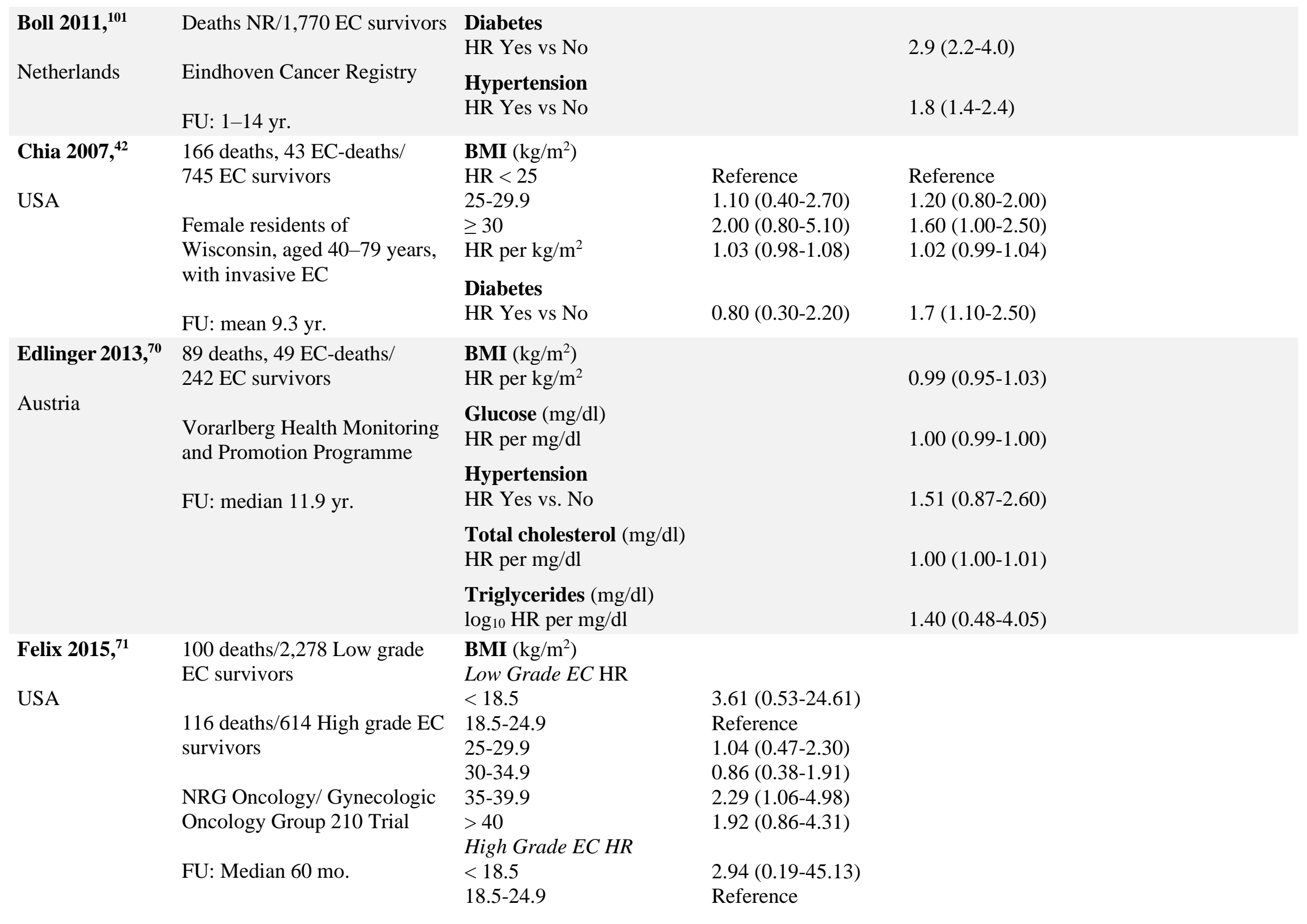




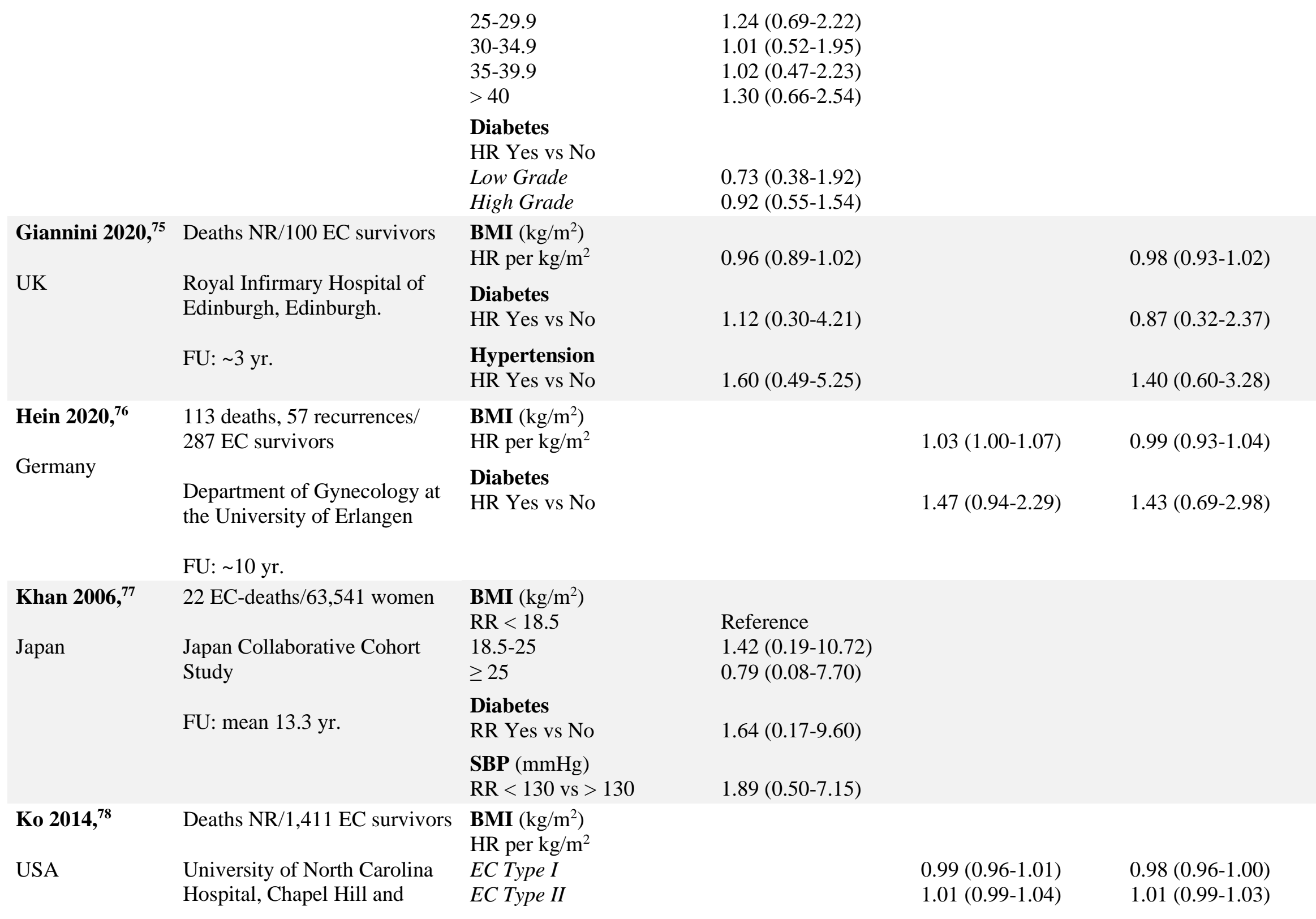




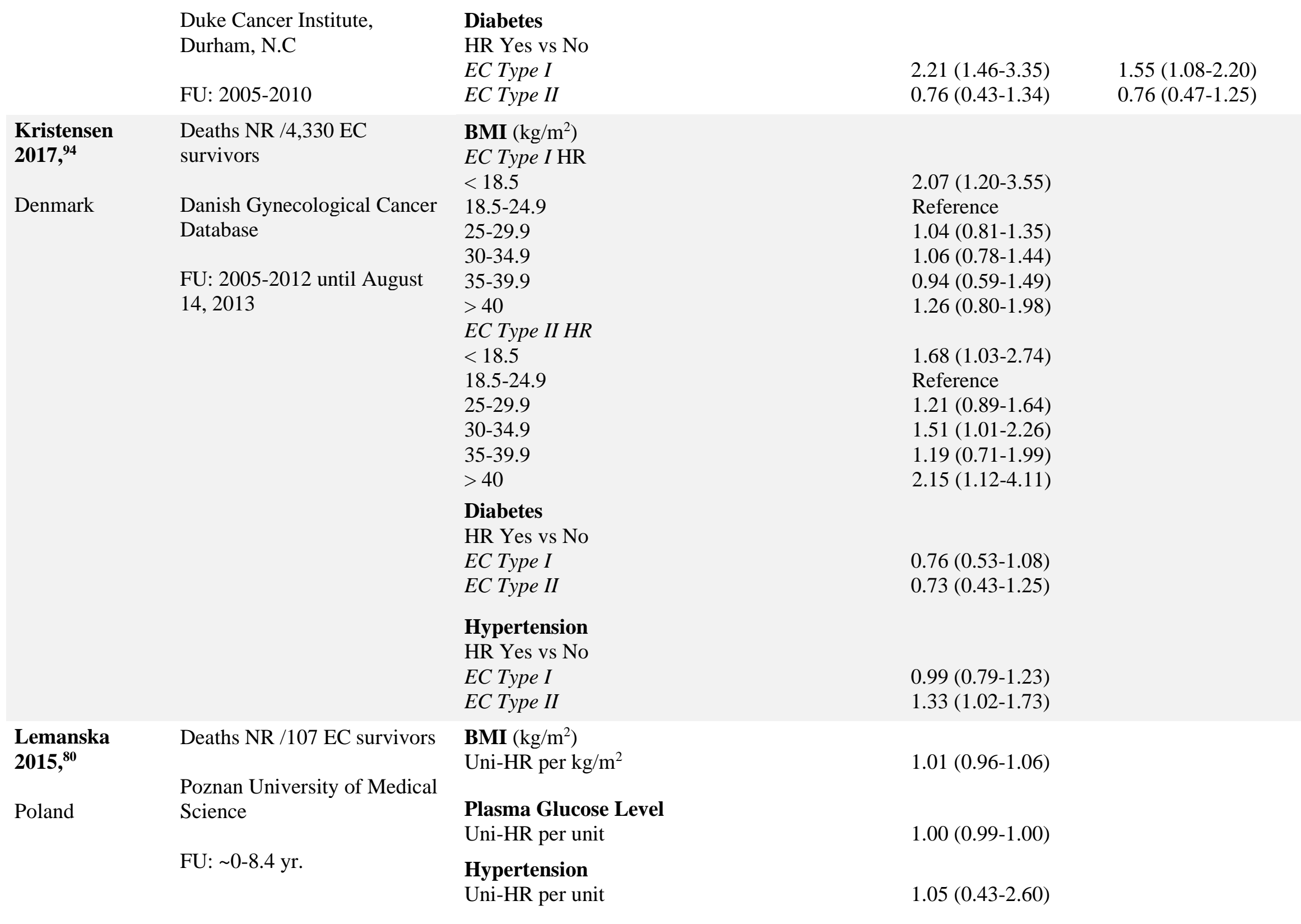




\begin{tabular}{|c|c|c|c|c|c|}
\hline Lindemann & 166 deaths, 56 EC-deaths/ & BMI $\left(\mathrm{kg} / \mathrm{m}^{2}\right)$ & & & \\
\hline $2015,{ }^{79}$ & 337 EC survivors & $\mathrm{HR}<25$ vs $>25$ & $1.47(0.69-3.11)$ & & \\
\hline & & $\mathrm{HR}<20$ & & $0.66(0.08-5.43)$ & \\
\hline Norway & Health Surveys in North- & $20-24.9$ & & Reference & \\
\hline & Trondelag & $25-29.9$ & & $1.10(0.69-1.75)$ & \\
\hline & & $30-34.9$ & & $1.10(0.65-1.88)$ & \\
\hline & FU: median 6.7 yr. & $\geq 35$ & & $1.34(0.78-2.32)$ & \\
\hline & & Diabetes & & & \\
\hline & & HR Yes vs No & $2.62(1.07-6.43)$ & $2.14(1.26-3.63)$ & \\
\hline & & $\mathrm{BMI}<25 \mathrm{~kg} / \mathrm{m}^{2}$ & & $6.35(1.90-21.14)$ & \\
\hline & & $\mathrm{BMI}>25 \mathrm{~kg} / \mathrm{m}^{2}$ & & $1.80(0.98-3.33)$ & \\
\hline Matsuo 2016, ${ }^{84}$ & 59 EC-deaths, 94 recurrences/ & BMI $\left(\mathrm{kg} / \mathrm{m}^{2}\right)$ & & & \\
\hline & 665 EC survivors & $\mathrm{HR}<30 \mathrm{vs} \geq 30$ & $1.19(0.62-2.28)$ & & $1.44(0.86-2.42)$ \\
\hline USA & & Diabetes & & & \\
\hline & Los Angeles County Medical & Uni-HR Yes vs No & $0.78(0.44-1.37)$ & & $0.88(0.57-1.37)$ \\
\hline & & Hypertension & & & \\
\hline & FU: median 36.4 mo. & Uni-HR Yes vs No & $0.98(0.58-1.63)$ & & $1.07(0.71-1.61)$ \\
\hline & & $\begin{array}{l}\text { Hypercholesterolemia } \\
\text { Uni-HR Yes vs No }\end{array}$ & $0.86(0.47-1.56)$ & & $1.04(0.66-1.64)$ \\
\hline Nagle 2018, 5 & 179 deaths, 123 EC-deaths & BMI $\left(\mathrm{kg} / \mathrm{m}^{2}\right)$ & & & \\
\hline & /1,359 EC survivors & HR 18.5-24.9 & Reference & Reference & \\
\hline Australia & & $25-29.9$ & $0.63(0.40-0.98)$ & $0.74(0.49-1.10)$ & \\
\hline & Australian National & $30-34.9$ & $0.68(0.38-1.23)$ & $0.86(0.53-1.40)$ & \\
\hline & Endometrial Cancer Study & $>35$ & $1.01(0.59-1.74)$ & $1.56(1.03-2.38)$ & \\
\hline & & per five $\mathrm{kg} / \mathrm{m}^{2}$ & $0.99(0.87-1.14)$ & $1.11(1.01-1.22)$ & \\
\hline & ro. mediant 7.1 ую. & Diabetes & & & \\
\hline & & HR Yes vs No & $2.09(1.31-3.35)$ & $2.54(1.72-3.74)$ & \\
\hline Nicholas 2014, ${ }^{41}$ & Deaths NR/ 490 EC survivors & BMI $\left(\mathrm{kg} / \mathrm{m}^{2}\right)$ & & & \\
\hline & & $\mathrm{HR}<25$ & & Reference & \\
\hline USA & Utah, USA & $25-39$ & & $1.41(0.83-2.40)$ & \\
\hline & & $>40$ & & $1.40(0.86-2.28)$ & \\
\hline
\end{tabular}


FU: median 4.5 yr.

Diabetes

HR Yes vs No

$1.58(1.07-2.33)$

Hypertension

HR Yes vs No

$1.51(1.06-2.15)$

\begin{tabular}{|c|c|}
\hline Olson 2012,95 & $\begin{array}{l}\text { 5,123 deaths, } 1,691 \text { EC-deaths } \\
12,568 \text { EC survivors }\end{array}$ \\
\hline USA & SEER-Medicare database \\
\hline & FU: median $3.5 \mathrm{yr}$. \\
\hline $\begin{array}{l}\text { Ruterbusch } \\
2014,{ }^{88}\end{array}$ & $\begin{array}{l}192 \text { deaths, } 43 \text { EC-deaths/ } \\
356 \text { White EC survivors }\end{array}$ \\
\hline USA & $\begin{array}{l}171 \text { deaths, } 66 \text { EC-deaths/ } \\
271 \text { Black EC survivors }\end{array}$ \\
\hline
\end{tabular}

Henry Ford Health System

\section{betes}

HR Yes vs No

White Women

$1.19(1.06-1.35)$

$1.27(1.18-1.36)$

Black Women

$0.97(0.73-1.30)$

$1.30(1.08-1.56)$

Hypertension

HR Yes vs No

White Women

Black Women

$1.07(0.96-1.19)$

$0.94(0.89-1.01)$

BMI $\left(\mathrm{kg} / \mathrm{m}^{2}\right)$

HR Yes vs No

White Women

$\geq 30 \mathrm{~kg} / \mathrm{m}^{2}$

$\geq 40 \mathrm{~kg} / \mathrm{m}^{2}$

Black Women

$\geq 30 \mathrm{~kg} / \mathrm{m}^{2}$

$0.76(0.55-1.05) \quad 0.74(0.60-0.92)$

FU: 1990-2005 till December 31, 2012

$\geq 40 \mathrm{~kg} / \mathrm{m}^{2}$

$\begin{array}{ll}0.89(0.44-1.79) & 1.04(0.75-1.43) \\ 0.72(0.24-2.15) & 1.33(0.86-2.06) \\ & \\ 0.81(0.44-1.51) & 1.04(0.69-1.56) \\ 1.04(0.53-2.02) & 1.07(0.70-1.63)\end{array}$

Diabetes

HR Yes vs No

White Women

$1.00(0.40-2.49) \quad 1.63(1.13-2.35)$

Black Women

$0.71(0.36-1.37)$

$1.17(0.80-1.71)$

Hypertension

HR Yes vs No

White Women

$0.47(0.23-0.98)$

$0.96(0.70-1.32)$

Black Women

$0.35(0.19-0.67) \quad 0.52(0.34-0.79)$ 


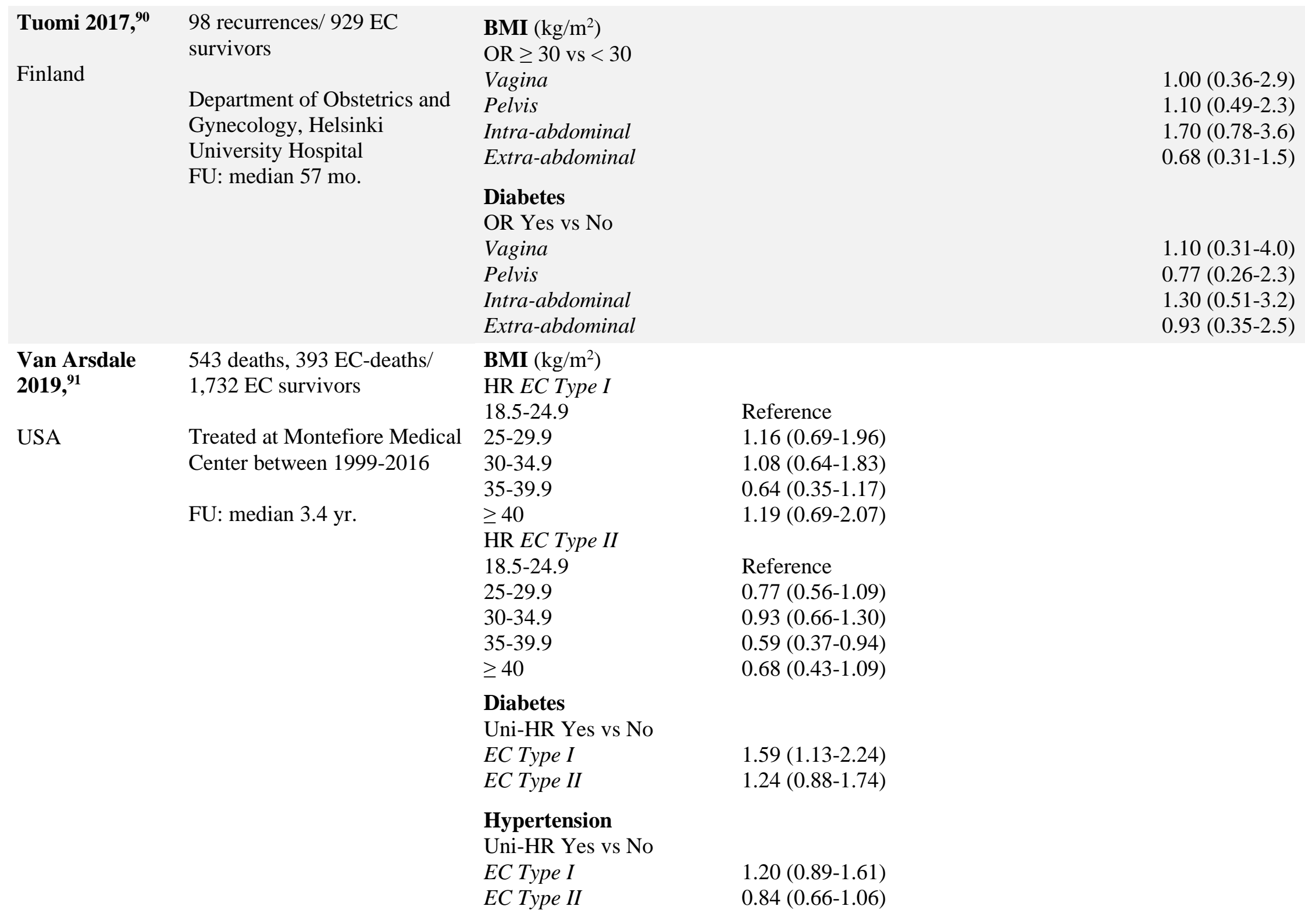




\section{Hyperlipidemia \\ Uni-HR Yes vs No \\ EC Type I \\ EC Type II \\ $0.76(0.56-1.01)$ \\ $0.55(0.43-0.71)$}

Diabetes

OR Yes vs No

$0.40(0.08-1.91)$

Hypertension

OR Yes vs No $3.51(1.03-11.90)$

Portal

FU: median 1.4 yr.

Zander 2012, ${ }^{40}$ Full cohort: 310 deaths/ 1,644 EC survivors

Netherlands †Sub-cohort: Deaths NR/ 388 EC survivors

\section{Diabetes}

HR Yes vs No $\quad 1.4(0.7-2.6) \dagger \quad 1.3(1.0-1.8)$

Hypertension

HR Yes vs No $\quad 0.9(0.7-1.3)$

Eindhoven Cancer Registry

FU: $\sim 5$ yr.

${ }^{a}$ Estimates have been presented as published or limited to two decimal places. Hazard ratio (HR), Univariable (Uni-), Odds ratio (OR), Not reported (NR), Endometrial cancer (EC), Systolic blood pressure (SBP), Diastolic blood pressure (DBP), Body Mass Index (BMI), Follow-up (FU), months (mo.) and years (yr.)

$\S$ Reciprocal of original estimate

+ Sub-cohort estimate 
Only four observational epidemiologic studies have previously examined the relationship between metabolic syndrome and survival outcomes among endometrial cancer survivors. Ni et al. ${ }^{56}$ used the presence of $\geq$ three of the following risk factors: Type II diabetes history, medically-treated hypertension, diagnosed or treated hyperlipidemia and abdominal obesity defined as either waist circumference $\geq 80 \mathrm{~cm}$ or BMI $\geq 28 \mathrm{~kg} / \mathrm{m}^{2}$ to represent the metabolic syndrome. This study observed 64 deaths among women living with histologically confirmed endometrial cancer $(\mathrm{n}=385)$ in China. ${ }^{56}$ Endometrial cancer survivors with metabolic syndrome had worse overall survival $(\mathrm{HR}=6.65,95 \% \mathrm{CI}=1.93-47.79)$ compared to those without the studyspecific metabolic syndrome definition. ${ }^{56}$ The same study-specific metabolic syndrome definition used by Ni et al. ${ }^{56}$ was also associated with an approximate twofold decrease in overall survival among 139 Chinese non-endometrioid uterine cancer patients. ${ }^{57}$

Jin et al. ${ }^{55}$ recently examined the association between metabolic syndrome and endometrial cancer-specific survival in the SEER-Medicare database. This database included 10,090 women diagnosed with endometrial cancer between 1992 and 2011 over the age of 65 years. Survivors with metabolic syndrome Medicare claim ("dysmetabolic syndrome") or claim codes for three of the five NCEP-ATP III metabolic syndrome criteria were considered to have this syndrome. ${ }^{55} \mathrm{~A}$ total of $1,612(16.0 \%)$ survivors met this criterion. This study ${ }^{55}$ noted that metabolic syndrome was associated with decreased endometrial cancer-specific survival among stage I/II survivors (HR=1.28, 95\% CI=1.09-1.53) but not survivors with stage III /IVa cancers $(\mathrm{HR}=1.18,95 \% \mathrm{CI}=0.93-1.49)$. Additionally, in the Metabolic Syndrome and Cancer Project (Me-Can), which collectively reported a total of 129 uterine corpus cancer deaths from a composite of six existing prospective cohort studies from Austria, Norway and Sweden, Bjorge et al. ${ }^{38}$ observed that survivors with metabolic syndrome had $1.56(95 \% \mathrm{CI}=1.32-1.84)$ times the 
hazard of endometrial cancer-specific death per one-unit increment of composite metabolic syndrome Z-score. However, only $58 \%$ of the 129 women who died of endometrial cancer were reported to have a prior diagnosis of incident endometrial cancer. ${ }^{38}$ To date, no known study has assessed the association between metabolic syndrome and recurrence outcomes among endometrial cancer survivors.

\subsection{Metabolic Syndrome Components and Endometrial Cancer Survival}

\subsubsection{Central Adiposity and Obesity}

\subsubsection{Overall Survival}

There is no known literature that directly assessed the relationship between measured central adiposity (i.e. waist circumference) and overall survival among endometrial cancer survivors, therefore the following discussion is limited to BMI. A systematic review and metaanalysis of 18 cohort studies conducted by Secord et al. ${ }^{99}$ observed a dose-response relationship between BMI and survival that reached statistical significance for women $\geq 40 \mathrm{~kg} / \mathrm{m}^{2}$ compared to $<25 \mathrm{~kg} / \mathrm{m}^{2}(\mathrm{OR}=1.66,95 \% \mathrm{CI}=1.10-2.51)$. Additionally, this study reported an inverse doseresponse relationship between increasing BMI and reduced survival $\left(\mathrm{OR}_{\text {per }} 10 \% \mathrm{BMI}=1.09,95 \%\right.$ $\mathrm{CI}=1.03-1.16$ ) for women living with endometrial cancer. ${ }^{99}$

In more recent studies, the literature regarding the association between BMI and overall survival remains mixed. Seventeen studies assessed the association with BMI at or prior to endometrial cancer diagnosis and overall survival, ${ }^{5,41,64,65,67,70,74,76,78-81,83,84,87,88,94}$ fourteen of which observed a null associations between BMI and survival. ${ }^{41,65,67,70,74,76,78-81,83,84,87,88}$ Of note, several of these estimates were derived from studies with small samples $(\mathrm{n}<250){ }^{70,74,80,87}$ However, Benedetti Panici et al. ${ }^{64}$ did observe a twofold decrease in overall survival for 
endometrial cancer survivors over the age of 65 years who were $\geq 30 \mathrm{~kg} / \mathrm{m}^{2}$ compared to $<30$ $\mathrm{kg} / \mathrm{m}^{2}$. Results from the Australian National Endometrial Cancer Study also suggested that endometrial cancer survivors with a pre-diagnosis BMI $>35 \mathrm{~kg} / \mathrm{m}^{2}$ had a reduced overall survival compared to survivors with a BMI within the recommended range $\left(18.5-24.9 \mathrm{~kg} / \mathrm{m}^{2}\right) .^{5}$ Additionally, a dose-response relationship per five $\mathrm{kg} / \mathrm{m}^{2}$ increase in pre-diagnosis BMI with reduced overall survival was observed in that study. ${ }^{5}$ The association with overall survival for endometrial cancer survivors did not differ by post-diagnosis BMI in the Women's Health Initiative $^{62}$ while the NIH-AARP Diet and Health Study observed a dose-response relationship (p-trend $=0.02$ ) for increased post-diagnosis BMI and decreased overall survival. ${ }^{61}$

When endometrial cancer was stratified by histologic type, Kristensen et al. ${ }^{94}$ observed that underweight (at diagnosis BMI $<18.5 \mathrm{~kg} / \mathrm{m}^{2}$ ) women living with Type I as well as Type II endometrial cancer had reduced survival relative to their normal weight $\left(18.5-24.9 \mathrm{~kg} / \mathrm{m}^{2}\right)$ counterparts. The authors reported no difference in overall survival among Type I endometrial cancer survivors living with Class I $\left(30-34.9 \mathrm{~kg} / \mathrm{m}^{2}\right)$, II $\left(35-39.9 \mathrm{~kg} / \mathrm{m}^{2}\right)$ or III $\left(>40 \mathrm{~kg} / \mathrm{m}^{2}\right)$ obesity. ${ }^{94}$ However, women living with Type II endometrial cancer were estimated to have reduced overall survival with increasing levels of obesity. ${ }^{94}$ In a second study that stratified the study population by endometrial cancer type, no significant relationship between overall survival and continuous BMI was observed. ${ }^{78}$

\subsubsection{Endometrial Cancer-Specific Survival}

The majority of studies that examined the association between obesity and endometrial cancer-specific survival $^{5,38,42,55,59,60,64-66,71,75,77,79,84,85,88,89,91}$ have been conducted in an American context. Both categorical and continuous results from the Women's Health Initiative ${ }^{59}$ and NIH- 
AARP Study ${ }^{60}$ suggest that increased pre-diagnosis BMI is negatively associated with endometrial cancer-specific survival. Specifically, endometrial cancer survivors living with Class I $\left(30-34.9 \mathrm{~kg} / \mathrm{m}^{2}\right)$ or II/III (> $\left.35 \mathrm{~kg} / \mathrm{m}^{2}\right)$ obesity had $2.06(95 \% \mathrm{CI}=1.01-4.2)$ and $2.23(95 \%$ $\mathrm{CI}=1.09-4.54, \mathrm{p}$-trend $=0.02)$ times the hazard of endometrial cancer-specific death compared to survivors with a BMI $<25 \mathrm{~kg} / \mathrm{m}^{2}$, respectively. ${ }^{59}$ Moreover, the five-year and ten-year endometrial cancer-specific survival decreased with greater pre-diagnosis BMI in the NIHAARP Study (five-year: p-trend $=0.04$, ten-year: p-trend $=0.02$ ) ${ }^{60}$ Bjorge et al..${ }^{38}$ Calle et al.${ }^{66}$ and Felix et al. ${ }^{71}$ also noted that elevated BMI was associated with decreased endometrial cancer-specific survival. In particular, Calle and colleagues ${ }^{66}$ observed that, in comparison to survivors with a BMI of $18.5-24.9 \mathrm{~kg} / \mathrm{m}^{2}$, survivors living with a BMI of $25.0-29.9 \mathrm{~kg} / \mathrm{m}^{2}$ $(\mathrm{HR}=1.50,95 \% \mathrm{CI}=1.26-1.78) 30.0-34.9 \mathrm{~kg} / \mathrm{m}^{2}(\mathrm{HR}=2.53,95 \% \mathrm{CI}=2.02-3.18), 35.0-39.9$ $\mathrm{kg} / \mathrm{m}^{2}(\mathrm{HR}=2.77,95 \% \mathrm{CI}=1.83-4.18)$ and $\geq 40.0 \mathrm{~kg} / \mathrm{m}^{2}(\mathrm{HR}=6.25,95 \% \mathrm{CI}=3.75-10.42$, $\mathrm{p}$-trend $<0.01)$ had reduced endometrial cancer-specific survival in the Cancer Prevention Study II. Conversely, Felix et al. ${ }^{71}$ noted that Type I but not Type II survivors living with obesity had worse endometrial cancer-specific survival compared to normal weight survivors (18.5-24.9 $\mathrm{kg} / \mathrm{m}^{2}$, p-trend $\left.<0.01\right)$.

In contrast, the Australian National Endometrial Cancer Study ${ }^{5}$ reported better endometrial cancer-specific survival was noted for those with a BMI of $25-29.9 \mathrm{~kg} / \mathrm{m}^{2}$ relative to normal weight survivors $\left(18.5-24.9 \mathrm{~kg} / \mathrm{m}^{2}\right)$, even though no differences in endometrial cancerspecific survival was observed in this cohort for those with Class I (30-34.9 $\left.\mathrm{kg} / \mathrm{m}^{2}\right)$ or II/III (> 35 $\mathrm{kg} / \mathrm{m}^{2}$ ) obesity. Van Arsdale et al. ${ }^{91}$ also reported improved endometrial cancer-specific survival among endometrial cancer survivors with Class II $\left(35-39.9 \mathrm{~kg} / \mathrm{m}^{2}\right)$ obesity compared to normal weight $\left(18.5-24.9 \mathrm{~kg} / \mathrm{m}^{2}\right)$ survivors. ${ }^{91}$ However, when stratified by endometrial cancer type, this 
association was only observed among women living with Type II endometrial cancer. ${ }^{91}$ Nine additional studies that assessed the association between pre/at-diagnosis BMI and endometrial cancer-specific survival failed to observe a statistically significant relationship. ${ }^{42,55,64,65,75,84,85,88,89}$ Univariate analyses and small sample sizes may have contributed to the null results seen in some of these studies. ${ }^{64,75,77}$

\subsubsection{Recurrence}

The association between BMI and endometrial cancer recurrence was investigated by fifteen studies. ${ }^{63,67-69,72,74-76,78,82-84,86,90,92}$ In a French Multicenter Study, Canlorbe et al. ${ }^{68}$ assessed the relationship between BMI and recurrence according to the European Society of Medical Oncology (ESMO) risk groups. The authors reported that in the high-risk group $(n=51)$, endometrial cancer survivors with a BMI $\geq 35 \mathrm{~kg} / \mathrm{m}^{2}$ had worse disease-free survival compared to survivors with a $\mathrm{BMI}<35 \mathrm{~kg} / \mathrm{m}^{2}(\mathrm{HR}=12.5,95 \% \mathrm{CI}=3.1-51.3)$. No association was observed in the low and intermediate ESMO risk groups. ${ }^{68}$ Moreover, none of the other studies that assessed the association between BMI and endometrial cancer recurrence observed a statistically significant relationship in the main ${ }^{63,67,75,76,82-84,90,92}$ or stratified analyses by endometrial cancer type, ${ }^{69,78}$ tumour histological subtype, ${ }^{72}$ or cancer stage. ${ }^{72,74,86}$

\subsubsection{Hyperglycemia and Diabetes}

\subsubsection{Overall Survival}

Two studies previously assessed the association between pre-diagnosis blood glucose levels and overall survival among endometrial cancer survivors, reporting no statistically significant associations. ${ }^{70,80}$ Seventeen studies ${ }^{5,40-42,76,78-80,88,94,95,100-102,105,106,109}$ additionally 
investigated the relationship between pre-/at-diagnosis diabetes and overall survival among women living with endometrial cancer. Six of these studies reported that endometrial cancer survivors with pre-diagnosis diabetes had between a 1.3- and 2.9-fold decrease in overall survival compared to survivors without diabetes. ${ }^{5,40-42,101,105}$ Olson et al. ${ }^{95}$ and Ruterbusch et al. ${ }^{88}$ also reported similar estimates for White endometrial cancer survivors, while only Olson et al. ${ }^{95}$ observed a statically significant association among Black endometrial cancer survivors.

In the Health Surveys in North-Trondelag (HUNT) study, diabetes was assessed within strata of BMI $\left(<25 \mathrm{~kg} / \mathrm{m}^{2}, \geq 25 \mathrm{~kg} / \mathrm{m}^{2}\right) \cdot{ }^{79}$ Among participants with a BMI $<25 \mathrm{~kg} / \mathrm{m}^{2}$, diabetes was associated with worse overall survival, while the association was not statistically significant for participants with a BMI $\geq 25 \mathrm{~kg} / \mathrm{m}^{2} \cdot{ }^{79} \mathrm{Four}^{76,80,100,109}$ additional studies observed no association between pre/at-diagnosis diabetes and overall survival among endometrial cancer survivors. Moreover, in the Echantillon Généraliste de Bénéficiaires database, Larouzée and colleagues ${ }^{106}$ noted that survivors with diabetes had improved three-year survival compared to those without diabetes.

Two studies stratified the overall survival estimates by endometrial cancer type and an additional study was comprised of only Type II endometrial cancer survivors. ${ }^{78,94,102}$ Ko and colleagues $^{78}$ reported that diabetes among women living with Type I endometrial cancer was associated with a twofold decrease in overall survival while no association was observed in the Danish Gynecological Cancer Database. ${ }^{94}$ Neither study observed a relationship between diabetes and overall survival among Type II endometrial cancer survivors. ${ }^{78,94}$ However, Burghau et al. ${ }^{102}$ observed a negative association between having a history of diabetes and overall survival amongst 108 uterine sarcomas and malignant mixed Mullerian tumour survivors $(\mathrm{HR}=2.81,95 \% \mathrm{CI}=1.13-6.98)$. 


\subsubsection{Endometrial Cancer-Specific Survival}

Seventeen studies to date have focused on the relationship between diabetes and endometrial cancer-specific survival, ${ }^{5,40,42,55,71,75,77,79,84,88,91,95,103-105,107,108}$ while a single article has investigated this association with blood glucose concentrations specifically. ${ }^{38}$ Neither the association between quintile of fasting blood glucose levels $(\mathrm{mmol} / \mathrm{L})$ or continuous glucose Zscore with endometrial cancer-specific survival in the Me-Can project reached statistical significance in the fully adjusted analyses. ${ }^{38}$

In the Cancer Prevention Study-II, Campbell and colleagues ${ }^{103}$ noted endometrial cancer survivors with diabetes had $1.33(95 \% \mathrm{CI}=1.08-1.65)$ times the hazard of endometrial cancerspecific death compared to survivors without diabetes. Ranc et al. ${ }^{108}$ also observed decrease endometrial cancer-specific survival among Danish endometrial cancer survivors with diabetes $(\mathrm{HR}=1.32,95 \% \mathrm{CI}=1.14-1.52)$. Reports from four additional studies found survivors with diabetes experienced between a twofold and threefold decrease in endometrial cancer-specific survival compared to survivors without diabetes. ${ }^{5,79,104,105}$ Five studies ${ }^{40,42,75,77,84}$ observed no association between diabetes and endometrial cancer-specific survival, although three $e^{42,75,77}$ of these studies had limited number of participants with diabetes or endometrial cancer-specific deaths.

Six studies assessed the association with diabetes and endometrial cancer-specific survival in stratified analysis. ${ }^{55,71,88,91,95,107}$ In the SEER-Medicare database, reduced endometrial cancer-specific survival was observed among White cancer survivors with diabetes ${ }^{95,107}$ while there was insufficient evidence that Black endometrial cancer survivors with diabetes compared to those without diabetes had decreased endometrial cancer-specific survival. ${ }^{95}$ Diabetes was not 
associated with endometrial cancer-specific survival in White or Black survivors in the Henry Ford Health System tumour registry, yet the number of individuals with known diabetes status in this study was limited (diabetes, White: $n=81$, Black: $n=78) .{ }^{88}$ Decreased endometrial cancerspecific survival was noted for Type I but not Type II endometrial cancer survivors with diabetes, ${ }^{91}$ whereas null associations were observed for analysis stratified by histological subtype $^{71}$ and cancer stage. ${ }^{55}$

\subsubsection{Recurrence}

Six studies assessed the association between diabetes and endometrial cancer recurrence. ${ }^{75,76,78,84,90,100}$ Ko et al. ${ }^{78}$ noted increased disease-free survival among Type I $(\mathrm{HR}=1.55,95 \% \mathrm{CI}=1.08-2.20)$ but not Type II endometrial cancer survivors with diabetes. ${ }^{78}$

Conversely, four other studies ${ }^{75,76,84,100}$ observed insufficient evidence of an association between diabetes status and disease-free survival. Moreover, the odds of site-specific recurrence did not differ by diabetes status for endometrial cancer survivors treated at the Helsinki University Hospital, Finland..$^{90}$

\subsubsection{Hypertension}

\subsubsection{Overall Survival}

It remains unclear whether or not an association between hypertension and overall survival among endometrial cancer survivors exists. A 1.5- to 1.8-fold decrease in overall survival for endometrial cancer survivors with hypertension compared to those without this condition has been noted in two studies. ${ }^{41,101}$ However, six additional studies failed to observe either crude ${ }^{80,111}$ or adjusted ${ }^{40,63,70,110}$ associations between hypertension and overall survival in 
this population. Furthermore, in stratified analyses, two studies ${ }^{88,95}$ reported no associations with hypertension and survival among White endometrial cancer survivors, while one study ${ }^{88}$ observed improved overall survival with hypertension among Black endometrial cancer survivors. Hypertension was also associated with worse overall survival among Type II but not Type I endometrial cancer survivors in the Danish Gynecological Cancer Database. ${ }^{94}$ A second study of 57 women with uterine carcinosarcomas (i.e. Type II) also observed a relationship between hypertension and reduced overall survival $(\mathrm{HR}=3.51,95 \% \mathrm{CI}=1.03-11.90) .{ }^{43}$

\subsubsection{Endometrial Cancer-Specific Survival}

Bjorge et al. ${ }^{38}$ failed to observe a relationship between continuous blood pressure Zscore, quintile of SBP, or quintile of DBP with endometrial cancer-specific survival, although there was some evidence of a linear trend for increasing quintile of DBP ( $\mathrm{p}$-trend <0.01). ${ }^{38}$ Amongst studies that assessed the relations between endometrial cancer-specific survival and dichotomous hypertension diagnoses, six studies ${ }^{75,77,84}$ reported null associations including three that failed to observe statistically significant estimates in cancer type, ${ }^{91}$ cancer stage,${ }^{55}$ and race ${ }^{95}$ stratified analyses. Only Ruterbusch et al. ${ }^{88}$ reported a significant relationship between hypertension diagnosis and endometrial cancer-specific survival in which both White and Black endometrial cancer survivors with hypertension had improved cancer-specific survival.

\subsubsection{Recurrence}

Three studies ${ }^{63,75,84}$ have assessed the association between hypertension and endometrial cancer recurrence. Two of these studies did not observe a difference in disease-free survival between normotensive and hypertensive endometrial cancer survivors. ${ }^{75,84}$ In contrary, Akbayir 
et al. ${ }^{63}$ reported a protective association between hypertension and endometrial cancer recurrence among 194 endometrial cancer survivors in Turkey. ${ }^{63}$

\subsubsection{Dyslipidemia}

\subsubsection{Overall Survival}

Dyslipidemia generally refers to elevated blood triglycerides, total cholesterol, low density lipoprotein cholesterol (LDL) and/or reduced HDL cholesterol levels. ${ }^{39}$ While elevated blood triglycerides and reduced HDL cholesterol levels are specifically included in the metabolic syndrome criteria, only one study ${ }^{70}$ has investigated the associations between triglycerides and total cholesterol with survival outcomes among endometrial cancer survivors, reporting no statistically significant associations.

\subsubsection{Endometrial Cancer-Specific Survival}

Limited evidence is available for the association between triglycerides and HDL cholesterol with endometrial cancer-specific survival. Three studies reported null associations between hypercholesterolemia, ${ }^{38,84}$ dyslipidemia $^{55}$ as well as hypertriglyceridemia ${ }^{38,55}$ with endometrial cancer-specific survival. Conversely, Van Arsdale et al. ${ }^{91}$ reported a null association between hyperlipidemia and endometrial cancer-specific survival among women living with Type I endometrial cancer but improved endometrial cancer-specific survival for Type II endometrial cancer survivors. 


\subsubsection{Recurrence}

Only Matuso et al. ${ }^{84}$ reported the association between dyslipidemia and endometrial cancer recurrence. The authors observed 94 recurrences among 665 endometrial cancer survivors treated at the Los Angeles County Medical Center, USA. ${ }^{84}$ Approximately $25 \%$ of the survivors had hypercholesterolemia at the time of diagnosis, although no association between hypercholesterolemia and disease-free survival was noted in the univariable analysis $(\mathrm{HR}=1.04,95 \% \mathrm{CI}=0.66-1.64) .{ }^{84}$

\subsection{Literature Summary}

Overall, there is some epidemiologic evidence that metabolic syndrome is associated with increased overall ${ }^{56,57}$ and endometrial cancer-specific survival. ${ }^{38,55}$ This literature has largely relied on self-reported cardiometabolic conditions and proxy metabolic syndrome component criteria. To date, the majority of studies have focused on the associations between pre- or atdiagnosis BMI and diabetes with survival outcomes among endometrial cancer survivors. Given that BMI does not differentiate between fat-free mass and fat mass, this measurement does not fully capture the complex physiology of excess body fat associated with cardiometabolic risk. ${ }^{14,112}$ Waist circumference is a strong indicator of visceral adiposity that has been positively associated with cardiometabolic disease and may better reflect the pathophysiology of excess body fatness. ${ }^{112}$ Moreover, literature regarding the associations between hypertension, elevated triglycerides and cholesterol with recurrence and survival outcomes among endometrial cancer survivors remains sparse. Therefore, additional studies are needed to improve understanding regarding the associations between metabolic syndrome and its components with cancer recurrence and survival outcomes among endometrial cancer survivors. 


\section{CHAPTER THREE: METHODS}

\subsection{Study Design and Population}

A prospective cohort of endometrial cancer survivors diagnosed between 2002 and 2006 who participated in a population-based case-control study in Alberta, Canada were followed until March 2019 for recurrence and survival outcomes. ${ }^{113}$ Survivors with histologically confirmed, invasive endometrial cancer were originally identified by the Alberta Cancer Registry, which has

a case ascertainment rate of $\geq 95 \% .{ }^{114}$ Women who were residents of Alberta, English speaking, between 30 and 80 years of age, able to complete an interview and diet history questionnaire, and had no prior history of cancer except non-melanoma skin cancer were eligible to participate. A total of 1,090 cases were identified and 900 cases met the abovementioned study inclusion criteria. Permission to contact cases from their referring physician was obtained for 808 women. Of those, $249(30.8 \%)$ declined to participate, seven $(0.9 \%)$ could not be contacted and three had complex atypical hyperplasia rather than endometrial cancer. ${ }^{113}$ A total of 549 endometrial cancer survivors were included in the original cancer case-control study. Nine additional participants were excluded from the cohort follow-up due to unsatisfactory baseline interviews $(n=7)$, cancer misclassification $(n=1)$ and being diagnosed with an ineligible endometrial cancer sub-type (neuroendocrine endometrial cancer, $\mathrm{n}=1$ ). The remaining 540 participants were included in this analysis.

Ethical approval for the study was obtained from the Health Research Ethics Board of Alberta- Cancer Committee (Alberta Cancer Board), the Conjoint Health Research Ethics Board (University of Calgary) and the Health Research Ethics Board (University of Alberta). All participants provided informed, written consent at baseline that included the follow-up for this cohort study. 


\subsection{Data Collection}

\subsubsection{Participant Characteristics}

Participants' demographic information, medical and medication use history, reproductive history, family history of cancer, and lifetime smoking habits were collected via an intervieweradministered questionnaire. Dietary caloric intake and lifetime alcohol consumption as well as lifetime physical activity participation were measured with the Canadian Diet Health Questionnaire-I and the Lifetime Total Physical Activity Questionnaire, respectively. ${ }^{115,116}$ Interviews occurred on average 22 weeks ( \pm 11.5 weeks $)$ following endometrial cancer diagnosis, $72 \%$ of interviews were completed within six months and $96 \%$ of participants completed the interview within 12 months of diagnosis. Interviewers were located in Calgary and Edmonton, Alberta and had extensive training in cognitive interviewing methods. ${ }^{117}$ These methods have been shown to improve participants' long-term recall. ${ }^{117}$ Detailed interviewer and coding manuals, as well as periodic monitoring of the interviews and regular meetings were in place for quality assurance purposes. Interview data were directly entered by the study staff into laptop computers using Blaise version 7 software (BLAISE®, Westat Corporation).

\subsubsection{Anthropometric Measurements}

All anthropometric measurements were taken at the time of the interview in the participants' homes. Direct measurements of the participants' height, waist and hip circumferences were taken to the first decimal point $(\mathrm{cm})$ using a Lufkin measuring tape. Waists and hips were defined as the widest part of the abdomen and buttocks, respectively. Participants' 
weight $(\mathrm{kg})$ was measured using a digital scale. All measurements were taken in multiple and an average of the measurements were used.

\subsubsection{Fasting Blood Draws}

Blood draws were taken after a minimum eight-hour fast at any participating clinic in the Calgary, Capital, Chinook or Palliser Health Care Regions. Participants enrolled in the study prior to hysterectomy had additional blood samples collected just prior to their surgery (presurgical $n=235$ ). For participants enrolled after hysterectomy, fasting blood draws were completed at a separate laboratory clinic visit four to six weeks following surgery (post-surgical $\mathrm{n}=286$ ). All participants had two 7-ml Vacutainer tubes containing ethylenediaminetetraacetic acid (EDTA; for plasma samples) and one 5-ml tube without additive (for serum samples) drawn. Blood samples were labelled with study IDs, date and time of the draw and transported on ice to the specimen biorepository at the Tom Baker Cancer Centre in Calgary, Alberta. Participants' samples were split between two ultra-low temperature $\left(-80{ }^{\circ} \mathrm{C}\right)$ freezers for storage to safeguard against sample loss in the event of a malfunction.

Laboratory assays were conducted between February 2008 and July 2009 in Dr. David WC Lau's laboratory at the University of Calgary by a technician blinded to the case-control status of the samples. Blood samples were analysed in batches with a 2:1 ratio of control to case samples based on the date of blood collection. Additionally, each batch included four quality assurance specimens: 1) a randomly selected case sample from the batch, 2) a randomly selected control sample from the batch, 3) a pooled case-control sample, and 4) a pooled sample of clinical serum specimen from women with a similar age range as the participants. The analyses of relevant biomarkers for the metabolic syndrome were performed in duplicate with 
colorimetric or absorbance-based assays (enzyme linked immunosorbent assay: ELISA) specific to the biomarker. The analyses of glucose (10009582; BioAssay Systems), triglycerides (10010303; BioAssay Systems), and HDL cholesterol (K614; Bio-Assay Systems) were completed with serum samples for all participants except three for whom serum samples were unavailable and plasma was used instead. The mean intra- and inter-batch coefficients of variation for fasting glucose were $3.7 \%$ and $4.6 \%$, for triglycerides were $1.9 \%$ and $3.5 \%$, and for HDL cholesterol were $4.3 \%$ and $5.5 \%$, respectively.

\subsubsection{Medical Chart Abstraction and Vital Status Assessment}

Clinical information including date of diagnosis, cancer histology and stage, primary and adjuvant treatment(s) received, cancer recurrence and occurrence of a new primary cancer diagnosis were abstracted from medical charts between 2006 and 2019 by the Alberta Cancer Registry trained Health Record Technicians. Overall tumour, lymph node, metastasis (TNM) stage was coded according to the American Joint Committee on Cancer guidelines. ${ }^{118}$ Cancer grade was categorized by the FIGO standards and was assessed by the study pathologist Dr. Anthony Magliocco. Additional cancer characteristics including myometrial or vascular invasion, lymph nodes involvement, presence of distant metastasis and site were available for 510 participants with tissue samples. The Alberta Cancer Registry obtained vital status and underlying cause of death data through regular record linkages with Vital Statistics Alberta and Statistics Canada during study follow-up. Vital status information for participants who died in provinces other than Alberta was obtained by periodic data linkages between provincial cancer registries and the Canadian Mortality Database. Additionally, for participants who left Alberta 
with unknown vital status, the date of leaving the province was considered the end of follow-up. Vital status was followed until death or March 20, 2019, whichever occurred first.

\subsection{Data Analysis}

\subsubsection{Variable Definitions}

Metabolic syndrome was defined with the harmonized definition using country- and sexspecific waist circumference criteria. ${ }^{46}$ An alternate hypertension criterion was used since direct blood pressure measurements were not available from the original case-control study. Participants who self-reported hypertension and/or hypertension medication use within one year of endometrial cancer diagnosis met this alternate criterion. Participants with partially missing data $(\mathrm{n}=27)$ who still met three or more metabolic syndrome components criteria were classified as having metabolic syndrome $(\mathrm{n}=7)$. Moreover, the number of metabolic syndrome components were grouped as zero or one, two or three, and four or five. Participants with incomplete metabolic syndrome data were assigned the lowest possible number of metabolic syndrome components based on the available data.

The medication alternative harmonized metabolic syndrome definition was also assessed. In this alternative definition, participants taking medication for diabetes or triglycerides within a year of diagnosis were also considered to meet the fasting blood glucose and blood triglycerides criteria, respectively. Specifically, participants with fasting blood glucose levels $\geq 100 \mathrm{mg} / \mathrm{dL}$ or taking medication for diabetes (e.g. acarbose, acetohexamide, chlorpropamide, gliclazide, glyburide, insulin, metformin hydrochloride, tolbutamide) within a year of the diagnosis date met the alternate glucose criterion. Women who took medication for gestational diabetes that did not persist after the end of pregnancy $(\mathrm{n}=2)$ were excluded from this alternative criterion. Moreover, 
survivors with blood triglycerides $\geq 150 \mathrm{mg} / \mathrm{dL}$ or using medication intended to lower

LDL/Triglycerides (e.g. atorvastatin, fluvastatin, lovastatin, pravastatin, simvastatin, gemfibrozil) within a year of the reference date met the blood triglycerides criterion. Since the year of hypertriglyceridemia diagnosis was not recoded, the year of high cholesterol diagnosis and duration of medication use was used as a proxy.

Endometrial cancer tumour and treatment characteristics were categorized into overall TNM stage (I, II, III/IV), FIGO grade (I/II, III, unknown/non-applicable) and primary cancer treatment(s). Women with incomplete overall cancer stage $(n=8)$ were coded as stage I based on available lymph node inclusion and metastatic information. FIGO tumour grade I/II were those with less than 50\% solid growth pattern, while grade III tumours had more than 50\% solid growth pattern. Tumours not classically graded (i.e. those within polyps, carcinosarcomas etc.) were coded as non-applicable while endometrial cancer survivors without tissue review (i.e. did not provide consent or did not have hysterectomy) had unknown tumour grade. Non-applicable and unknown tumour grades were combined and treated as a separate category to grade I/II and III. Participants were categorized by the type of primary cancer treatment(s) received: 1) hysterectomy at baseline, 2) hysterectomy and completed chemotherapy, 3) hysterectomy and completed radiation therapy, 4) hysterectomy and at least two types of adjuvant treatments (chemotherapy, radiation and/or hormone therapy), and 5) missing treatment. Of note, no participants received hormone therapy without also receiving chemotherapy or radiation therapy. Missing treatment was defined as any primary treatment (hysterectomy, chemotherapy, radiation or hormonal therapy) not received because of health complications, participants refusing treatment or participants not completing treatment. A new primary cancer was considered any 
metachronous new primary cancer recoded by the Alberta Cancer Registry six or more months following initial endometrial cancer diagnosis.

Participants baseline demographic and medical history information were defined as follows: baseline age (years), measured BMI (continuous; $\mathrm{kg} / \mathrm{m}^{2}$ ), marital status (married/common law, other), race (White, non-White), highest education attained ( $\leq$ high school, non-university certificate, university degree), residency (rural, urban), parity (null, onetwo, $\geq$ two pregnancies $\geq 20$ weeks gestation), menopausal status (pre- or peri-menopausal, postmenopausal), hormone treatment status (ever, never), first degree family history of uterine (Not otherwise specified (NOS)) or colorectal cancer (yes, no/missing), lifetime recreational physical activity (metabolic-equivalent task [MET]-hours/week/year), lifetime alcohol consumption (grams/year), dietary caloric intake (total daily food energy intake (kcal) excluding implausible values (i.e. $<600 \mathrm{kcal}$ or $>5000 \mathrm{kcal}$ )), smoking status (ever, never: smoked 100 cigarettes in their life), smoking pack years for current and ex-smokers was defined as: (number of cigarettes smoked daily)*(duration of smoking in years)/ 20 , and the number of major comorbidities diagnosed before endometrial cancer diagnosis (zero, one, $\geq$ two of any of the following: angina pectoris, pulmonary embolism, myocardial infarction, stroke, thrombosis). Type II diabetes, high cholesterol, hypertension or other free text comorbidity responses were not included in the variable created for the number of major comorbidities.

Cancer recurrence was considered as any primary site recurrence or progression, lymph node recurrence or metastasis of the primary cancer noted in the participants medical record. Deaths attributable to endometrial or uterine (NOS) cancer based on the International Classification of Diseases for Oncology, Third Edition (C54-C55) were categorized as endometrial cancer-specific deaths. ${ }^{11}$ Survival time was calculated as the time from the initial 
diagnosis of endometrial cancer until death or March 20, 2019, whichever occurred first. The endpoints for disease-free survival, overall survival and endometrial cancer-specific survival were the first recurrence or death from any cause, death from any cause and death attributable to endometrial cancer, respectively. The time to recurrence was defined as the time from initial endometrial cancer diagnosis to the date of first recurrence, all other events were censored.

\subsubsection{Statistical Analyses}

Descriptive statistics were used to assess the distribution and/or proportion of metabolic syndrome, metabolic syndrome components, covariates and outcome events. Dietary caloric intake and race information were not included in multivariable analysis because of the reduction to the analytical sample size due to missing/implausible information $(n=489)$ and a nearly homogeneous sample, respectively. Cox proportional hazards regression models were used to estimate multivariable-adjusted $\mathrm{HR}$ and $95 \% \mathrm{CI}$ for the associations between metabolic syndrome (yes, no), the number of metabolic syndrome components (zero-one, two-three, fourfive) and individual metabolic syndrome components with disease-free survival, overall survival and recurrence outcomes. Fine and Gray competing risk models were used for the endometrial cancer-specific survival outcome. The proportional hazards assumption was evaluated with visual and statistical assessments of Schoenfeld residuals and Wald-tests for the interaction of the exposure variable and time in the Cox models and Fine and Gray models, respectively. Models that did not satisfy the proportional hazards assumption were further assessed in an exploratory five- and ten-year restricted time analyses.

The individual metabolic syndrome components tested were: waist circumference $(\geq 88$ $\mathrm{cm}$, per five $\mathrm{cm}$ ), fasting blood glucose levels ( $\geq 100 \mathrm{mg} / \mathrm{dL}$, per five $\mathrm{mg} / \mathrm{dL}$ ), blood triglycerides 
( $\geq 150 \mathrm{mg} / \mathrm{dL}$, per five $\mathrm{mg} / \mathrm{dL}$ ), HDL cholesterol ( $<50 \mathrm{mg} / \mathrm{dL}$, per five $\mathrm{mg} / \mathrm{dL}$ ), and selfreported hypertension (yes, no). Continuous exposures were centered at dichotomous cut-points. Endometrial cancer stage, grade, primary cancer treatment(s), baseline age (years) and non-linear age $\left(\mathrm{age}^{2}\right)$ variables were forced into the model based on a priori biological plausibility. Additionally, disease-free survival models were adjusted for the time to first new primary cancer, overall survival models were adjusted for the time to first recurrence/new primary cancer, and endometrial cancer-specific survival models were adjusted for the time to first recurrence. Finally, all metabolic syndrome component models were mutually adjusted for the other metabolic syndrome risk factors.

All other potential confounding variables were included in preliminary models and removed sequentially via backwards elimination. Only variables that meaningfully changed the estimate of the association of interest remained in the models. A change greater than roughly ten percent was generally considered meaningful. The number of major comorbidities (zero, one, $\geq$ two) was included in metabolic syndrome models for disease-free and overall survival outcomes while continuous BMI $\left(\mathrm{kg} / \mathrm{m}^{2}\right)$ was included in all multivariable-adjusted models. There was insufficient evidence that lifetime alcohol consumption, marital status, hormone treatment status, parity, menopausal status, smoking pack years, highest education attained, residency, or firstdegree family history of uterine (NOS) or colorectal cancer confounded the associations of interest. Nor was there evidence that fasting blood insulin levels $(\mathrm{pM} / \mathrm{ml})$ confounded the associations in fasting blood glucose models.

Potential modification of the associations between metabolic syndrome and its components with overall and disease-free survival by BMI $\left(\mathrm{kg} / \mathrm{m}^{2}\right)$ and lifetime recreational physical activity (MET-hours/week/year) were assessed with Wald-tests in hierarchically well- 
formulated models. Associations with statistically significant effect modification were presented by median groups.

Sample size for this analysis was predetermined by the number of available cases; hence, the final analytical sample included participants with complete metabolic syndrome data $(n=520)$ and complete metabolic syndrome component data $(\mathrm{n}=513)$. Statistical significance was set at five percent and all analyses were performed using Stata software version 15.1 (StataCorp LLC. College Station, TX). 


\section{CHAPTER FOUR: RESULTS}

This chapter includes the manuscript from this thesis which addresses the relationship between the harmonized metabolic syndrome criteria and its components with disease-free survival, overall survival, endometrial cancer-specific survival and recurrence among endometrial cancer survivors from the Alberta Endometrial Cancer Cohort Study.

The citation for the manuscript is:

Kokts-Porietis RL, McNeil J, Nelson GS, Courneya KS, Cook LS, Friedenreich CM.

Prospective Cohort Study of Metabolic Syndrome and Endometrial Cancer Survival. Gynecol Oncol. 2020. https://doi.org/10.1016/j.ygyno.2020.06.488 
4.1. Prospective Cohort Study of Metabolic Syndrome and Endometrial Cancer Survival 4.1.1. Abstract

Objective: Comorbidities are known to increase endometrial cancer risk, but the separate and combined impact of these risk factors on endometrial cancer survival remains unclear. This study aimed to determine the associations between metabolic syndrome and its components with disease-free survival, overall survival, endometrial cancer-specific survival and recurrence among endometrial cancer survivors.

Methods: Cases from a population-based case-control study who were diagnosed with primary endometrial cancer between 2002 and 2006 in Alberta, Canada were followed until death or March 20, 2019. Baseline in-person interviews, direct anthropometric measurements and fasting blood samples were used to assess metabolic syndrome (presence of $\geq 3$ of the following: waist circumference $\geq 88 \mathrm{~cm}$, fasting blood glucose $\geq 100 \mathrm{mg} / \mathrm{dL}$, triglycerides $\geq 150 \mathrm{mg} / \mathrm{dL}$, highdensity lipoprotein cholesterol $<50 \mathrm{mg} / \mathrm{dL}$ and self-reported hypertension). Cox proportional hazards regression and Fine and Gray competing risk models were used to estimate multivariateadjusted hazard ratios (95\% CI) for these associations.

Results: Among 540 endometrial cancer survivors, 325 had metabolic syndrome at diagnosis and $132 \mathrm{had}$ a recurrence and/or died during the median 14.2 years of follow-up (range: 0.3-16.5 years). In multivariable analyses, being diagnosed with metabolic syndrome (HR=1.98, 95\% $\mathrm{CI}=1.07-3.67)$ and having an elevated waist circumference $(\geq 88 \mathrm{~cm} ; \mathrm{HR}=2.12,95 \% \mathrm{CI}=1.18$ 3.80; $\mathrm{HR}_{\mathrm{per}} 5 \mathrm{~cm}=1.21,95 \% \mathrm{CI}=1.07-1.36$ ) were associated with worse overall survival. Additionally, increasing waist circumference (per $5 \mathrm{~cm}$ ) was also associated worse with diseasefree survival $\left(\mathrm{HR}_{\mathrm{per}} 5 \mathrm{~cm}=1.11,95 \% \mathrm{CI}=1.00-1.24\right)$. 
Conclusion: The metabolic syndrome, in particular central adiposity, were associated with worse overall and disease-free survival in endometrial cancer survivors.

Keywords: Endometrial Cancer, Survival, Metabolic Syndrome, Co-morbidities 


\subsubsection{Introduction}

Endometrial cancer is the sixth most frequently diagnosed cancer among women worldwide. ${ }^{1}$ In 2018, the International Agency for Research on Cancer (IARC) estimated that 382,069 new cases of endometrial cancer and 89,929 endometrial cancer-specific deaths occurred globally ${ }^{1}$ with the highest rates occurring in North America and Central and Eastern Europe. ${ }^{1,9}$ Between 2005 and 2015, uterine cancer mortality rates increased $2.0 \%$ annually in Canada and $1.9 \%$ annually between 2011 and 2015 in the USA, making endometrial cancer one of the few cancer types with both increasing incidence and mortality rates. ${ }^{11,12}$

Obesity and diabetes have been previously identified as major risk factors for endometrial cancer, whereas physical activity is thought to reduce endometrial cancer risk..$^{9,36,39}$ These risk factors may also be associated with endometrial cancer recurrence and mortality. $5,41,99$ Specifically, obesity is an established risk factor for endometrial cancer survival, with convincing biological plausibility through metabolic disruptions, hormonal and growth factor imbalances, unopposed estrogens released by adipose tissue ${ }^{14}$ and chronic inflammation that prompts tumour growth. ${ }^{9,14-16}$ Furthermore, among cancer survivors, obesity has been associated with reduced responses to standard treatments, higher rates of metastatic disease and poorer prognoses compared to normal weight individuals. ${ }^{16}$ Diabetes and hypertension may also increase cancer-specific mortality and all-cause mortality in this population. ${ }^{5,40-42,94}$ However, the combined impact of these comorbidities on endometrial cancer survival outcomes remains unclear.

Metabolic syndrome is defined as the clustering of cardiovascular and metabolic disease risk factors including central adiposity, hypertension, hyperglycemia, elevated triglycerides and low high-density lipoprotein (HDL) cholesterol. ${ }^{46}$ Women with metabolic syndrome are 
estimated to have a roughly twofold elevation in endometrial cancer risk compared to those without. ${ }^{3,4,39,52,58,119}$ Despite biological plausibility that metabolic syndrome may also be associated with endometrial cancer recurrence and survival, ${ }^{14,54}$ limited information on survival outcomes currently exists. Therefore, the objective of the current study was to determine the association between metabolic syndrome and its components, defined by harmonized criteria ${ }^{46}$ with disease-free survival, overall survival, endometrial cancer-specific survival and recurrence among endometrial cancer survivors.

\subsubsection{Methods}

\subsubsection{Study Design}

The Alberta Endometrial Cancer Cohort Study is a follow-up of women diagnosed with histologically confirmed, invasive, primary endometrial cancer between 2002 and 2006 who participated in a population-based case-control study in Alberta, Canada. ${ }^{113}$ Full details of the case-control study have been previously reported. ${ }^{58,113}$ Briefly, $549(61.0 \%)$ cases identified through the Alberta Cancer Registry who were residents of Alberta, English speaking, between 30-80 years of age, able to complete an interview and diet history questionnaire, and had no prior history of cancer except non-melanoma skin cancer, were included. Nine participants were excluded from this analysis due to unsatisfactory baseline interviews $(n=7)$, cancer misclassification $(n=1)$ and being diagnosed with an ineligible endometrial cancer sub-type (neuroendocrine endometrial cancer; $n=1$ ). The remaining 540 participants $(60.0 \%)$ were included in this study. Ethical approval for the study was obtained from the Alberta Cancer Board, the Conjoint Health Research Ethics Board (University of Calgary) and the Health 
Research Ethics Board (University of Alberta). All participants provided informed, written consent.

\subsubsection{Data Collection}

Participant's demographic information, menstrual and reproductive history, hormone use history, medical history, medication use information, family history of cancer, and lifetime smoking habits were collected via an interviewer-administered questionnaire following endometrial cancer diagnosis (mean $22 \pm 11.5$ weeks). ${ }^{113}$ Lifetime alcohol consumption and lifetime physical activity participation were measured with the Canadian Diet Health Questionnaire-I and the Lifetime Total Physical Activity Questionnaire (LTPAQ), respectively. ${ }^{115,116}$ At the time of the interviews, standardized anthropometric measurements including the participant's height, weight and waist circumferences were taken in triplicate and the average was recorded as the final measurement. ${ }^{113}$ Participants fasted for at least eight hours before blood samples were collected either pre-hysterectomy or four-six weeks posthysterectomy if blood could not be drawn pre-surgery (pre-surgical $n=235$; post-surgical $n=286$ ). Blood samples were processed into blood fractions (serum, plasma, red blood cells, and buffy coat), frozen at $-80^{\circ} \mathrm{C}$ within 24 hours of collection and stored in biorepository at the Tom Baker Cancer Centre, Calgary, Alberta. ${ }^{120}$

\subsubsection{Follow-up and Outcome Ascertainment}

Clinical information including date of diagnosis, cancer histology and stage, primary and adjuvant treatment(s) received, cancer recurrence and occurrence of a new primary cancer diagnosis were abstracted from medical charts by the Alberta Cancer Registry trained Health 
Record Technicians. Overall stage (tumour, lymph node, metastasis (TNM)) was coded according to the American Joint Committee on Cancer guidelines. ${ }^{118}$ Women with incomplete overall cancer TNM stage $(\mathrm{n}=8)$ were coded based on available lymph node inclusion and metastatic information. Cancer grade (International Federation of Gynecology and Obstetrics) and tumour histology (endometrioid, mixed cell, serous, clear cell, mucinous adenocarcinoma, carcinosarcoma, adenosarcoma and other) were additionally available via tissue samples, as

previously described. ${ }^{120}$ Vital status and underlying cause of death data were provided by Vital Statistics Alberta and Statistics Canada, respectively. Vital status information from participants who died in provinces other than Alberta were obtained from data linkages between provincial cancer registries.

For the purpose of this analysis, cancer recurrence was considered as any primary site recurrence or progression, lymph node recurrence or metastasis of the primary cancer noted in the medical record. Deaths that were attributable to endometrial or uterine (NOS) cancer were categorized as endometrial cancer-specific deaths. Survival time was calculated as the time from the initial diagnosis of endometrial cancer until death or March 20, 2019, whichever occurred first. Disease-free survival was defined as the time from diagnosis to the first recurrence or death from any cause, while overall survival was the time from diagnosis until the time of death from any cause.

\subsubsection{Statistical Analyses}

Metabolic syndrome was categorized with the harmonized definition using country- and sex-specific waist circumference criteria. ${ }^{46}$ Participants with three or more of the following risk factors were classified as having metabolic syndrome: waist circumference $\geq 88 \mathrm{~cm}$, fasting 
blood glucose levels $\geq 100 \mathrm{mg} / \mathrm{dL}$, blood triglycerides $\geq 150 \mathrm{mg} / \mathrm{dL}$, HDL cholesterol $<50 \mathrm{mg} / \mathrm{dL}$, or self-reported hypertension and/or hypertension medication use within one year of endometrial cancer diagnosis. The alternate hypertension criterion was used as direct blood pressure measurements were not available from the original case-control study. ${ }^{46,58}$ Twenty-seven participants were missing one or more metabolic syndrome components (missing: waist circumference $n=4$; fasting blood glucose levels $n=20$; blood triglycerides $n=23$; HDL cholesterol $n=20$ ). Participants with partial missing data who still met three or more metabolic syndrome components criteria were classified as having metabolic syndrome $(\mathrm{n}=7)$.

Cox proportional hazards regression models were used to estimate multivariable-adjusted hazard ratios (HR) and 95\% confidence intervals $(95 \% \mathrm{CI}$ ) for the associations between metabolic syndrome (yes, no), the number of metabolic syndrome components $(0-1,2-3,4-5)$ and individual metabolic syndrome components with disease-free survival, overall survival and recurrence outcomes. Fine and Gray competing risk models were used for the endometrial cancer-specific survival outcome. The individual metabolic syndrome components tested were: waist circumference ( $\geq 88 \mathrm{~cm}$, per $5 \mathrm{~cm}$ ), fasting blood glucose levels ( $\geq 100 \mathrm{mg} / \mathrm{dL}$, per 5 $\mathrm{mg} / \mathrm{dL}$ ), blood triglycerides ( $\geq 150 \mathrm{mg} / \mathrm{dL}$, per $5 \mathrm{mg} / \mathrm{dL})$, HDL cholesterol $(<50 \mathrm{mg} / \mathrm{dL}$, per 5 $\mathrm{mg} / \mathrm{dL}$ ), and self-reported hypertension (yes, no). Continuous exposures were centred at dichotomous cut-points. All models were hierarchically well-formulated and adjusted for endometrial cancer stage (I, II, III/IV), grade (I/II, III, unknown/non-applicable), primary cancer treatment(s) (hysterectomy, hysterectomy/chemotherapy, hysterectomy/radiation, hysterectomy/ chemotherapy/radiation and/or hormone therapy, missing treatment), baseline age (years) and non-linear age $\left(\mathrm{age}^{2}\right)$ based on a priori biological plausibility. Additionally, disease-free survival models were adjusted for the time to first new primary cancer, overall survival models were 
adjusted for the time to first recurrence/new primary cancer, and endometrial cancer-specific survival models were adjusted for the time to first recurrence. Moreover, all metabolic syndrome component models were mutually adjusted for the other metabolic syndrome risk factors.

Additional confounding variables were identified via backwards elimination. Continuous BMI $\left(\mathrm{kg} / \mathrm{m}^{2}\right)$ was included in all multivariable-adjusted models while there was only evidence that the number of major comorbidities $(0,1, \geq 2$ of any of the following: angina pectoris, pulmonary embolism, myocardial infarction, stroke, thrombosis) confounded the association between metabolic syndrome with disease-free and overall survival. There was insufficient evidence that lifetime alcohol consumption (grams/year), marital status (married/common law, other), hormone replacement treatment status (ever, never), parity (null,1-2, $\geq 2$ ) menopausal status (pre or peri-menopausal, post-menopausal), smoking pack years defined as: (number of cigarettes smoked daily $) *($ duration of smoking in years)/ 20, highest education attained ( $\leq$ high school, non-university certificate, university degree), residency (rural, urban) or first degree family history of uterine (NOS) or colorectal cancer (yes, no/missing) confounded the associations of interest. Nor was there evidence that fasting blood insulin levels $(\mathrm{pM} / \mathrm{ml})$ confound associations in fasting blood glucose models.

Potential modification of the association between metabolic syndrome and its components with overall and disease-free survival by BMI $\left(\mathrm{kg} / \mathrm{m}^{2}\right)$ and lifetime recreational physical activity (metabolic-equivalent task [MET]-hours/week/year) were assessed with Waldtests. Associations with statistically significant effect modification are presented by median groups.

The proportional hazards assumption was evaluated with visual and statistical assessments of Schoenfeld residuals and Wald-tests for the interaction of the exposure variable 
and time in the Cox models and Fine and Gray models, respectively. Results are not presented for the analyses that did not satisfy the proportional hazards assumption. Sample size for this analysis was predetermined by the number of available cases; hence, the final analytical sample included participants with complete metabolic syndrome data $(n=520)$ and complete metabolic syndrome component data ( $n=513$; Figure 2). All analyses were performed using Stata software version 15.1 (StataCorp LLC. College Station, TX).

\subsubsection{Results}

Among the 540 endometrial cancer survivors, 325 (60.2\%) had metabolic syndrome at the time of endometrial cancer diagnosis and 132 had a recurrence and/or died during the median 14.2 years of follow-up (range: 0.3-16.5 years). Specifically, there were 73 recurrences, 50 endometrial cancer-specific deaths and 111 overall deaths during follow-up. Most participants were diagnosed with endometroid histology $(81.5 \%)$, low stage and grade endometrial cancer (Stage I: 77.8\%; Grade I: 53.3\%), had hysterectomy as part of primary treatment (97.6\%) and a mean age of 59.1 (SD 9.3) years (Table 3).

In the multivariable-adjusted analyses, a statistically significant association between metabolic syndrome and worse overall survival $(\mathrm{HR}=1.98,95 \% \mathrm{CI}=1.07-3.67)$ was observed (Table 4). The association with metabolic syndrome and endometrial cancer-specific survival did not reach statistical significance $(\mathrm{HR}=1.80,95 \% \mathrm{CI}=0.75-4.33)$. Likewise, there was insufficient evidence of associations between metabolic syndrome with disease-free survival or endometrial cancer recurrence in these analyses (Table 4).

For the individual components of metabolic syndrome, waist circumference $(\geq 88 \mathrm{~cm})$ was associated with statistically significantly worse overall survival $(\mathrm{HR}=2.12,95 \% \mathrm{CI}=1.18$ - 
3.80) but not disease-free survival $(\mathrm{HR}=1.66,95 \% \mathrm{CI}=0.98-2.79)$, endometrial cancer-specific survival $(\mathrm{HR}=2.14,95 \% \mathrm{CI}=0.73-6.31)$ or recurrence $(\mathrm{HR}=1.21,95 \% \mathrm{CI}=0.58-2.52)$. Positive linear dose-response relationships were observed per $5 \mathrm{~cm}$ increase in waist circumference with overall survival $\left(\mathrm{HR}_{\mathrm{per}} 5 \mathrm{~cm}=1.21,95 \% \mathrm{CI}=1.07-1.36\right)$ and disease-free survival $\left(\mathrm{HR}_{\mathrm{per}} 5 \mathrm{~cm}=1.11\right.$, 95\% CI=1.00-1.24) in the multivariable-adjusted models.

Lifetime recreational physical activity prior to diagnosis (MET-hours/week/year) significantly modified ( $\mathrm{p}$-value $<0.05$ ) the associations between metabolic syndrome, waist circumference (per $5 \mathrm{~cm}$ ), HDL cholesterol (per $5 \mathrm{mg} / \mathrm{dL}$ ) and hypertension with overall survival as well as the association between HDL cholesterol (per $5 \mathrm{mg} / \mathrm{dL}$ ) and disease-free survival in the multivariable-adjusted models. Specifically, among endometrial cancer survivors with lower lifetime recreational physical activity ( $<9.7$ MET-hours/week/year), those with metabolic syndrome had worse overall survival $(\mathrm{HR}=2.45,95 \% \mathrm{CI}=1.19-5.05)$ compared to participants without the syndrome. No association with metabolic syndrome and overall survival was observed amongst survivors with higher recreational physical activity (for $\geq 9.7$ METhours/week/year, $\mathrm{HR}=0.88,95 \% \mathrm{CI}=0.46-1.69)$. Increasing waist circumference was associated with worse overall survival for women with lower $\left(\mathrm{HR}_{\mathrm{per}} 5 \mathrm{~cm}=1.30,95 \% \mathrm{CI}=1.13-1.49\right)$, as well as higher $\left(\mathrm{HR}_{\mathrm{per}} 5 \mathrm{~cm}=1.16,95 \% \mathrm{CI}=1.02-1.32\right)$ lifetime recreational physical activity. Improved overall survival $\left(\mathrm{HR}_{\mathrm{per}} 5 \mathrm{mg} / \mathrm{dL}=0.85,95 \% \mathrm{CI}=0.76-0.96\right)$ and disease-free survival $\left(\mathrm{HR}_{\mathrm{per}} 5\right.$ $\mathrm{mg} / \mathrm{dL}=0.85,95 \% \mathrm{CI}=0.76-0.94)$ was observed for greater HDL cholesterol levels (per $5 \mathrm{mg} / \mathrm{dL}$ ) among women with lower physical activity. Lastly, hypertension was associated with worse overall survival $(\mathrm{HR}=1.80,95 \% \mathrm{CI}=1.02-3.18)$ among endometrial cancer survivors with lower lifetime recreational physical activity. 
Finally, the models assessing the number of metabolic syndrome components and fasting blood glucose did not satisfy the proportional hazard assumption and are therefore not reported.

\subsubsection{Discussion}

In the current study, endometrial cancer survivors with metabolic syndrome had approximately a twofold decrease in overall survival compared to study participants who did not have this syndrome. Our findings also suggest that metabolic syndrome may be associated with worse endometrial cancer-specific survival although the association was not statistically significant in the multivariable-adjusted model. There was insufficient evidence of an association between metabolic syndrome and disease-free survival or endometrial cancer recurrence in the present study. Finally, of the individual metabolic syndrome components, only waist circumference was observed to be associated with survival outcomes.

To our knowledge, this investigation is the first study among endometrial cancer survivors to use a standardized definition of metabolic syndrome to test its association with survival outcomes as well as the first conducted in a North American context. Our findings are generally consistent with prior literature that observed worse overall and endometrial cancerspecific survival with metabolic syndrome among endometrial cancer survivors. Ni and colleagues $^{56}$ used a study-specific definition of metabolic syndrome (presence of $\geq$ three of the following components: history of Type 2 diabetes, medically-treated hypertension, diagnosed or treated hyperlipidemia and either waist circumference $\geq 80 \mathrm{~cm}$ or $\mathrm{BMI} \geq 28 \mathrm{~kg} / \mathrm{m}^{2}$ ) to assess the association with all-cause mortality among histologically confirmed endometrial cancer cases ( $n=385 ; n=64$ deaths) in China. The authors noted that endometrial cancer survivors with the study-specific metabolic syndrome had 6.65 (95\% CI=1.93-47.79) times the hazard of all-cause 
mortality compared to those without metabolic syndrome. ${ }^{56}$ The same study-specific metabolic syndrome definition used by $\mathrm{Ni}$ et al., ${ }^{56}$ was also associated with worse overall survival among 139 Chinese non-endometrioid uterine cancer patients. ${ }^{57}$ Within the Metabolic Syndrome and Cancer Project (Me-Can), metabolic syndrome was associated with 1.56 (95\% CI=1.32-1.84) times the hazard of uterine cancer-specific mortality per one-unit increment of composite metabolic syndrome Z-score. ${ }^{38}$

In the current study, women with a waist circumference above $88 \mathrm{~cm}$ had approximately half the overall survival compared to women with a waist circumference below $88 \mathrm{~cm}$. Furthermore, for every additional $5 \mathrm{~cm}$ increase in waist circumference above $88 \mathrm{~cm}$, women were estimated to have a $21 \%$ decrease in overall survival and an $11 \%$ decrease in disease-free survival. Our findings also suggest that greater waist circumference may also be associated with worse disease-free survival, endometrial cancer-specific survival and recurrence although these associations were attenuated by BMI. Previous research has shown that both waist circumference and BMI are positively correlated with estrogen levels in endometrial cancer survivors, unopposed estrogens released by adipose tissue is a known risk factor of endometrial cancer tumour proliferation. ${ }^{14,121}$ The underlying mechanism of pathogenesis via adipose tissue and elevated estrogen concentrations may partially explain why the associations between waist circumference with disease-free survival, endometrial cancer-specific survival and recurrence were attenuated by BMI. However, waist circumference is a strong indicator of central adiposity which has been positively associated with additional biological mechanisms related to hyperglycemia, dyslipidemia, hyperinsulinemia and systemic inflammation that may also drive tumour pathogenesis. ${ }^{14}$ Previous findings from the Nurses' Health Study observed that women had a $34 \%(95 \% \mathrm{CI}=23 \%-39 \%)$ increase in all-cause mortality and a $18 \%(95 \% \mathrm{CI}=9 \%-28 \%)$ 
increase in obesity-related (colon cancer, breast cancer, pancreatic cancer, uterine, ovarian, and kidney cancer) cancer mortality per $\sim 10 \mathrm{~cm}$ increase in waist circumference in multivariable adjusted models including adjustments for BMI. ${ }^{122}$

To our knowledge, the present study is the first to assess whether lifetime physical activity prior to diagnosis impacts the relationship between metabolic syndrome at diagnosis and its components with survival outcomes among endometrial cancer survivors. Our findings suggest that women with greater lifetime recreational physical activity participation do not experience the same harmful associations between metabolic syndrome, waist circumference, and hypertension with overall survival compared to those with lower amounts of lifetime physical activity participation. A protective association was also observed with increasing HDL cholesterol and survival outcomes amongst women with lower lifetime physical activity participation. These findings add to current literature that suggests cancer survivors with greater physical activity participation experience better survival, although specific estimates among endometrial cancer survivors are limited. ${ }^{19,123}$ Arem and colleagues previously reported an association between pre-diagnosis recreational moderate-to-vigorous-intensity physical activity and lower overall 5-year mortality, but not 10-year mortality, endometrial cancer-specific mortality or all-cause mortality in the Women's Health Initiative ${ }^{59}$ and the National Institutes of Health- American Association of Retired Persons (NIH-AARP) Diet and Health Study. ${ }^{60}$ This study has several key strengths, including a substantial study follow-up time of nearly 17 years. Participants were representative of the larger endometrial cancer survivor population living in Alberta since cases were originally identified through the Alberta Cancer Registry which has a $\geq 95 \%$ case ascertainment rate ${ }^{114}$ of histologically confirmed, endometrial cancer cases. Additionally, data linkage with national and provincial registries ensured that 
comprehensive vital status data were captured. Direct measurements of most of the metabolic syndrome components, particularly the anthropometric measures and blood assays, were assessed in multiple to provide valid and reliable data. Finally, covariates were extensively measured during the in-person interviews and interviewers were trained with cognitive interview methods.

Some limitations must be acknowledged in the current study. Primarily, the small sample size and limited number of events may have hindered the ability of the current study to observe statistically significant associations. Particularly the estimates of endometrial cancer-specific survival and recurrence should be interpreted with caution because of the small number of events in the dichotomized analyses. Larger studies are thus needed to confirm and contribute to our findings. Moreover, some results could not be presented because the proportional hazard assumptions were violated. The metabolic syndrome was only assessed at the time of cancer diagnosis and may not represent an individual's health status for the entire follow-up period. Consequently, the prevalence of metabolic syndrome may have been underestimated in this cohort. However, previous findings from the Atherosclerosis Risk in Communities Study suggest that, for the majority of individuals $(76 \%)$, the severity of metabolic syndrome increases over time. ${ }^{124}$ Moreover, medical treatment of individual risk factors failed to reduce the severity of metabolic syndrome during the follow-up period in the Atherosclerosis Risk in Communities Study. ${ }^{124}$ Therefore, women in the current study likely did not have improved metabolic heath status during follow-up if they had metabolic syndrome or some of its components at baseline. Finally, given that the study participants represent a largely homogenous group of White (93.9\%), well educated (67.2\% $\geq$ high school education), endometrial cancer survivors living in 
Alberta, the current findings may not be generalizable to all populations including racial-ethnic minority populations in Canada.

Given that endometrial cancer mortality rates are currently rising ${ }^{11,12}$ and women living with endometrial cancer have greater amounts of diagnosed, undiagnosed or undertreated cardiometabolic risk factors compared to the general population, ${ }^{6}$ identifying opportunities to intervene is becoming increasingly important. In this study, the results suggest that the association between metabolic syndrome and endometrial cancer survival is largely explained by central adiposity. Moreover, endometrial cancer survivors with greater lifetime recreational physical activity had improved survival despite metabolic syndrome risk factors compared to those with lower lifetime physical activity. Additional research is needed to confirm these results and improve our understanding of the role of physical activity and other lifestyle factors in modifying the metabolic syndrome and survival associations among endometrial cancer survivors. 


\subsubsection{Declarations}

Conflict of Interest Statement

The authors declare that there are no conflicts of interest.

\section{Acknowledgements}

Study design and initiation of data collection methods was done by Lisa Strosher and Pam

Round for data collection. Rita Biel, Aleata Ryhorchuk and Sana Fakih were the Study

Coordinators for the original case-control study (RB, AR) and for the cohort follow-up (RB, SF).

Interviews were done by Tamara Bellmont, Kay Christie, Pearl Cooke, Linda Davison, Carolyn Henderson, Tacey Lawrence, Rosalie Merkosky, Jodi Parrotta, Brenda Platzer, Cyndi Rasa, Nicole Slot, Keely Winnitoy and Carol-Anne Zawalykut. Farit Vakhetov did data management and Thomas Speidel created the variables in the case-control study that were used in the cohort follow-up. Qinggang Wang provided statistical analysis support.

\section{Funding Source}

Renée L. Kokts-Porietis held a Canada Graduate Scholarships Master's Award from the Canadian Institutes of Health Research and a Master's Research Scholarship from the University of Calgary. Christine M. Friedenreich received career awards from the Canadian Institutes of Health Research and the Alberta Heritage Foundation for Medical Research/Alberta Innovates (AHFMR/Alberta Innovates). Linda S. Cook and Kerry S. Courneya held Canada Research Chairs and Linda S. Cook also received career award funding from AHFMR and is support from NCI P30CA118100. This study was funded by three separate grants from the National Cancer Institute of Canada through the Canadian Cancer Society (NCIC Grants No. 12018, 13010, 17323) and from one grant awarded by the former Alberta Cancer Board (ACB Grant 22190). 
Author Contribution

Renée L. Kokts-Porietis: Conceptualization, Methodology, Formal analysis, Writing - Original Draft, Writing - Review \& Editing. Jessica McNeil: Conceptualization, Writing - Review \& Editing, Supervision. Gregg Nelson: Conceptualization, Writing - Review \& Editing, Supervision. Kerry S. Courneya: Conceptualization, Methodology, Funding acquisition, Investigation, Project administration, Writing - Review \& Editing. Linda S. Cook:

Conceptualization, Methodology, Funding acquisition, Investigation, Project administration, Writing - Review \& Editing. Christine M. Friedenreich: Conceptualization, Methodology, Investigation, Data Curation, Funding acquisition, Writing - Review \& Editing, Supervision. 


\subsubsection{Tables and Figures}

Table 3. Participant Characteristics of the Alberta Endometrial Cancer Cohort by Vital Status, Alberta, Canada (N=540)

\begin{tabular}{|c|c|c|c|c|c|}
\hline $\begin{array}{c}\text { Characteristics } \\
(\text { Mean (SD); N (\%)) }\end{array}$ & $\underset{\substack{\text { All } \\
(\mathbf{n}=540)}}{ }$ & $\begin{array}{c}\text { Alive } \\
(n=429)\end{array}$ & $\begin{array}{c}\text { Disease-Free } \\
\text { Survival } \\
(n=132)\end{array}$ & $\begin{array}{c}\text { Overall } \\
\text { Deaths } \\
(n=111)\end{array}$ & $\begin{array}{c}\text { Cancer-Specific } \\
\text { Deaths }(n=50)\end{array}$ \\
\hline Age at diagnosis (years) & $59.1(9.3)$ & $57.6(8.8)$ & $63.5(9.8)$ & $64.9(9.2)$ & $62.7(7.7)$ \\
\hline \multicolumn{6}{|l|}{ Highest Education } \\
\hline High school diploma & $177(32.8)$ & $134(31.2)$ & $50(37.9)$ & $43(38.7)$ & $15(30.0)$ \\
\hline Non-university certificate & $249(46.1)$ & $198(46.2)$ & $61(46.2)$ & $51(46.0)$ & $26(52.0)$ \\
\hline University degree & $114(21.1)$ & $97(22.6)$ & $21(15.9)$ & $17(15.3)$ & $9(18.0)$ \\
\hline Married/common-law & $372(68.9)$ & $300(69.9)$ & $84(63.6)$ & $72(64.9)$ & $30(60.0)$ \\
\hline White & $507(93.9)$ & $401(93.5)$ & $123(93.2)$ & $106(95.5)$ & $46(92.0)$ \\
\hline Urban residence & $363(67.2)$ & $300(69.9)$ & $77(58.3)$ & $63(56.8)$ & $32(64.0)$ \\
\hline \multicolumn{6}{|l|}{ Parity } \\
\hline 0 & $111(20.6)$ & $91(21.2)$ & $23(18.70)$ & $20(18.0)$ & $8(16.0)$ \\
\hline $1-2$ & $248(45.9)$ & $208(48.5)$ & $45(36.59)$ & $40(36.0)$ & $21(42.0)$ \\
\hline$>2$ & $181(33.5)$ & $130(30.3)$ & $55(44.72)$ & $51(46.0)$ & $21(42.0)$ \\
\hline Post-Menopausal & $415(76.9)$ & $312(72.7)$ & $116(87.9)$ & $103(92.8)$ & $45(90.0)$ \\
\hline \multicolumn{6}{|l|}{ Histology } \\
\hline Endometroid & $440(81.5)$ & $366(85.3)$ & $91(68.9)$ & $74(66.7)$ & $28(56.0)$ \\
\hline Non-Endometroid & $100(18.5)$ & $63(14.7)$ & $41(31.1)$ & $37(33.3)$ & $22(44.0)$ \\
\hline \multicolumn{6}{|l|}{ Overall AJCC stage } \\
\hline I & $428(79.3)$ & $362(84.4)$ & $78(59.1)$ & $66(59.5)$ & $17(34.0)$ \\
\hline II & $69(12.8)$ & $49(11.4)$ & $25(18.9)$ & $20(18.0)$ & $11(22.0)$ \\
\hline III/IV & $43(8.0)$ & $18(4.2)$ & $29(22.0)$ & $25(22.5)$ & $22(44.0)$ \\
\hline \multicolumn{6}{|l|}{ FIGO Grade } \\
\hline I & $288(53.3)$ & $250(58.3)$ & $47(35.6)$ & $38(34.2)$ & $10(20.0)$ \\
\hline II & $125(23.2)$ & $102(23.8)$ & $27(20.5)$ & $23(20.7)$ & $11(22.0)$ \\
\hline III & $73(13.5)$ & $41(9.6)$ & $36(27.3)$ & $32(28.8)$ & $18(36.0)$ \\
\hline Not Reported/Missing & $54(10.0)$ & $36(8.4)$ & $22(16.7)$ & $18(12.2)$ & $11(22.0)$ \\
\hline \multicolumn{6}{|l|}{ Primary treatment } \\
\hline Hysterectomy & $527(97.6)$ & $422(98.4)$ & $120(90.9)$ & $105(94.6)$ & $46(92.0)$ \\
\hline Chemotherapy & $45(8.3)$ & $26(6.1)$ & $20(15.2)$ & $19(17.1)$ & $17(34.0)$ \\
\hline Hormone therapy & $6(1.1)$ & $6(1.4)$ & $2(1.5)$ & $0(0)$ & $0(0)$ \\
\hline
\end{tabular}




$\begin{array}{llllll}\quad \text { Radiation therapy } & 168(31.1) & 124(28.9) & 52(39.4) & 44(39.6) & 21(42.0) \\ \quad \text { Not Received } & 33(6.1) & 16(3.7) & 25(18.9) & 17(15.3) & 12(24.0) \\ \text { New Primary Cancer } & 92(17.0) & 63(14.7) & 33(25.0) & 29(26.1) & 2(4.0) \\ \text { Family History of Uterine or } & 88(16.3) & 64(14.9) & 27(20.5) & 24(21.6) & 12(24.0) \\ \text { Colorectal Cancer } & 32.3(7.9) & 32.1(17.1) & 33.2(17.6) & 33.0(9.1) & 33.3(17.6) \\ \text { BMI (kg/m }) & 271(50.2) & 218(50.8) & 62(47.0) & 53(47.8) & 19(38.0) \\ \text { Smoker (Ever) } & 12.1(9.2) & 12.5(9.6) & 11.2(8.9) & 10.5(7.5) & 10.6(7.3) \\ \text { Lifetime recreational PA } & 325(60.2) & 248(57.8) & 87(65.9) & 77(69.4) & 37(74.0) \\ \text { (Mets-hours/week/year) } & & & & & \\ \text { Metabolic Syndrome } & & & & & \\ \text { Metabolic Syndrome } & 81(15.0) & 68(15.9) & 15(11.4) & 13(11.7) & 5(10.0) \\ \text { Components } & 114(21.1) & 98(22.8) & 23(17.4) & 16(14.4) & 6(12.0) \\ \quad 0-1 & 147(27.2) & 117(27.3) & 32(24.2) & 30(27.0) & 18(36.0) \\ 2 & 130(24.1) & 95(22.1) & 41(31.1) & 35(31.5) & 14(28.0) \\ 3 & 48(8.9) & 36(8.4) & 14(10.6) & 12(10.8) & 5(10.0) \\ 4 & 98.0(18.8) & 97.4(18.2) & 100.6(20.1) & 100.5(20.9) & 99.1(22.5) \\ \quad 5 & 122.6(43.4) & 123.2(44.1) & 120.1(38.7) & 120.4(40.4) & 118.3(38.5) \\ \text { Waist Circumference (cm) } & 146.8(97.9) & 143.8(97.5) & 161.6(102.7) & 158.2(98.7) & 154.6(87.8) \\ \text { Fasting Glucose (mg/dL) } & 37.3(12.7) & 37.9(12.4) & 34.9(13.7) & 35.2(13.8) & 36.3(13.9) \\ \text { Triglycerides (mg/dL) } & 186(34.4) & 133(31.0) & 57(43.2) & 53(47.8) & 22(44.0) \\ \text { HDL Cholesterol (mg/dL) } & & & & \\ \text { Hypertension (self-reported) } & & \end{array}$

${ }^{\mathrm{a} A J C C}$ : American Joint Committee on Cancer, FIGO: International Federation of Gynecology and Obstetrics, BMI: body mass index (kg/m²), PA: Physical Activity, HDL: High-Density Lipoprotein.

${ }^{b}$ Women with incomplete TMN stage $(\mathrm{n}=8)$ were categorized as Stage I. 
Table 4. Disease-Free Survival, Overall Survival, Endometrial Cancer-Specific Survival and Recurrence Outcomes for Endometrial Cancer Survivors in the Alberta Endometrial Cancer Cohort, Alberta, Canada (N=520)

\begin{tabular}{|c|c|c|c|c|c|c|c|c|}
\hline & \multicolumn{2}{|c|}{$\begin{array}{l}\text { Disease-Free Survival } \\
\qquad(\mathrm{n}=125 / 520)\end{array}$} & \multicolumn{2}{|c|}{$\begin{array}{l}\text { Overall Survival } \\
\qquad(\mathrm{n}=106 / 520)\end{array}$} & \multicolumn{2}{|c|}{$\begin{array}{c}\text { Endometrial Cancer- } \\
\text { Specific Survival }(n=48 / 520)\end{array}$} & \multicolumn{2}{|c|}{$\begin{array}{l}\text { Recurrence } \\
(\mathrm{n}=69 / 520)\end{array}$} \\
\hline & $\begin{array}{l}\text { Events } \\
\text { /Cases }\end{array}$ & $\begin{array}{c}\text { Multivariable } \\
\text { HR }(95 \% \text { CI })\end{array}$ & $\begin{array}{l}\text { Events } \\
\text { /Cases }\end{array}$ & $\begin{array}{c}\text { Multivariable } \\
\text { HR }(95 \% \text { CI) }\end{array}$ & $\begin{array}{l}\text { Events } \\
\text { /Cases }\end{array}$ & $\begin{array}{l}\text { Multivariable } \\
\text { HR }(95 \% \text { CI) }\end{array}$ & $\begin{array}{l}\text { Events } \\
\text { /Cases }\end{array}$ & $\begin{array}{c}\text { Multivariable } \\
\text { HR (95\% CI) }\end{array}$ \\
\hline \multicolumn{9}{|c|}{ Metabolic Syndrome } \\
\hline No & $38 / 195$ & 1.00 & $29 / 195$ & 1.00 & $11 / 195$ & 1.00 & $22 / 195$ & 1.00 \\
\hline Yes & $87 / 325$ & $1.05(0.68-1.63)$ & $77 / 325$ & $1.98(1.07-3.67)$ & $37 / 325$ & $1.80(0.75-4.33)$ & $47 / 325$ & $0.77(0.43-1.38)$ \\
\hline \multicolumn{9}{|c|}{ Waist Circumference (cm) } \\
\hline$<88$ & $32 / 168$ & 1.00 & $28 / 168$ & 1.00 & $13 / 168$ & 1.00 & $18 / 168$ & 1.00 \\
\hline$\geq 88$ & $90 / 345$ & $1.66(0.98-2.79)$ & $75 / 345$ & $2.12(1.18-3.80)$ & $34 / 345$ & $2.14(0.73-6.31)$ & $50 / 345$ & $1.21(0.58-2.52)$ \\
\hline Per $5 \mathrm{~cm}$ increase & & $1.11(1.00-1.24)$ & & $1.21(1.07-1.36)$ & & $1.09(0.91-1.31)$ & & $1.04(0.90-1.21)$ \\
\hline \multicolumn{9}{|c|}{ Fasting Glucose (mg/dL) } \\
\hline$<100$ & $44 / 177$ & 1.00 & $38 / 177$ & 1.00 & $17 / 177$ & 1.00 & $25 / 177$ & 1.00 \\
\hline$\geq 100$ & $78 / 336$ & NR & $65 / 336$ & NR & $30 / 336$ & $0.65(0.33-1.31)$ & $42 / 336$ & $0.77(0.46-1.30)$ \\
\hline Per $5 \mathrm{mg} / \mathrm{dL}$ increase & & $0.98(0.95-1.00)$ & & $0.98(0.96-1.01)$ & & NR & & $0.98(0.95-1.01)$ \\
\hline \multicolumn{9}{|l|}{ Triglycerides (mg/dL) } \\
\hline$<150$ & $69 / 331$ & 1.00 & $58 / 331$ & 1.00 & $24 / 331$ & 1.00 & $38 / 331$ & 1.00 \\
\hline$\geq 150$ & $53 / 182$ & $1.35(0.90-2.02)$ & $45 / 182$ & $1.32(0.83-2.10)$ & $23 / 182$ & $1.26(0.56-2.86)$ & $30 / 182$ & $1.08(0.61-1.89)$ \\
\hline Per $5 \mathrm{mg} / \mathrm{dL}$ increase & & $1.01(1.00-1.01)$ & & $1.00(0.99-1.01)$ & & $1.00(0.98-1.01)$ & & $1.00(0.98-1.01)$ \\
\hline \multicolumn{9}{|c|}{ HDL Cholesterol (mg/dL) } \\
\hline$\geq 50$ & $16 / 73$ & 1.00 & $14 / 73$ & 1.00 & $7 / 73$ & 1.00 & $9 / 73$ & 1.00 \\
\hline$<50$ & $106 / 440$ & $0.61(0.34-1.08)$ & $89 / 440$ & $0.55(0.29-1.03)$ & $40 / 440$ & $0.31(0.12-0.82)$ & $59 / 440$ & $0.63(0.29-1.34)$ \\
\hline Per $5 \mathrm{mg} / \mathrm{dL}$ increase & & $0.94(0.87-1.02)$ & & $0.96(0.88-1.04)$ & & $1.01(0.85-1.21)$ & & $0.95(0.85-1.06)$ \\
\hline \multicolumn{9}{|l|}{ Hypertension } \\
\hline No & $70 / 339$ & 1.00 & $54 / 339$ & 1.00 & $26 / 339$ & 1.00 & $44 / 339$ & 1.00 \\
\hline Yes & $52 / 174$ & $0.90(0.60-1.34)$ & $49 / 174$ & $1.18(0.77-1.81)$ & $21 / 174$ & $1.01(0.85-1.21)$ & $24 / 174$ & $0.55(0.31-1.00)$ \\
\hline
\end{tabular}




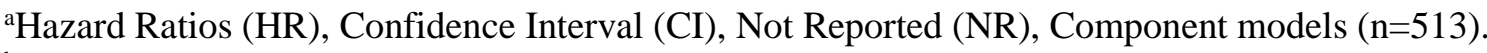

${ }^{\mathrm{b}}$ Multivariable models adjusted for stage (I/missing, II, III/IV), grade (I/II, III, missing), primary treatment(s) (hysterectomy,

hysterectomy/chemotherapy, hysterectomy/radiation, hysterectomy/chemotherapy/ radiation and/or hormone therapy, missing treatment), baseline age (years), non-linear age $\left(\mathrm{age}^{2}\right)$ and continuous BMI $\left(\mathrm{kg} / \mathrm{m}^{2}\right)$. Disease-free survival model additionally adjusted for time to first new primary cancer; Overall survival additionally adjusted for time to first new primary cancer/recurrence; Endometrial cancer-specific survival additionally adjusted for time to first recurrence.

${ }^{\mathrm{c}}$ All models for metabolic syndrome components were mutually adjusted for the other components.

dDisease-free survival and overall survival metabolic syndrome multivariable models were additionally adjusted for number of major comorbidities $(0,1, \geq 2)$.

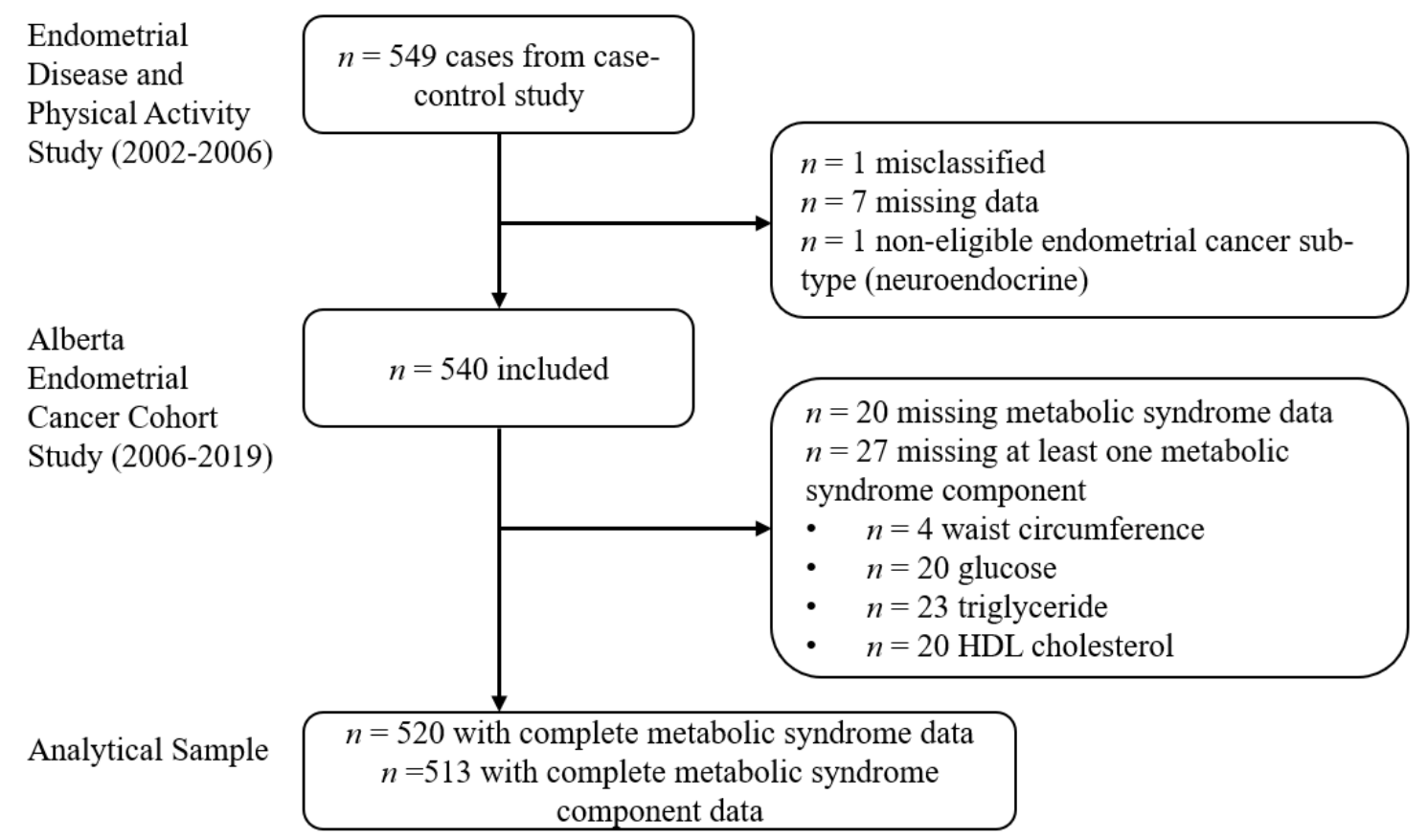

Figure 2. Participant Flowchart for the Alberta Endometrial Cancer Cohort, Alberta, Canada 


\subsection{Supplementary Results}

The results for the medication alternative metabolic syndrome definition analysis and exploratory five- and ten-year restricted analyses for models in the Prospective Cohort Study of Metabolic Syndrome and Endometrial Cancer Survival manuscript (Section 4.1.4) that did not satisfy the proportional hazards assumption are provided in the sections below.

\subsubsection{Medication Alternative Metabolic Syndrome Analysis}

In this analysis, 33 study participants used diabetes medication within a year of their endometrial cancer diagnosis date. Of these, 28 participants also had fasting blood glucose levels $\geq 100 \mathrm{mg} / \mathrm{dL}$ and thus already met the blood glucose criterion regardless of their medication use. Consequently, only five additional participants met the alternative blood glucose criterion in addition to the 340 participants with fasting blood glucose levels $\geq 100 \mathrm{mg} / \mathrm{dL}$ ( $\mathrm{n}=345$ ). Likewise, 22 participants who used triglyceride medication within a year of diagnosis met the alternate triglyceride criterion (triglyceride $\geq 150 \mathrm{mg} / \mathrm{dL} \mathrm{n}=183$, total $\mathrm{n}=205$ ). With the proxy fasting blood glucose and blood triglycerides criterion included in the medication alternative metabolic syndrome definition, $330(63.2 \%)$ of 521 participants were categorized as having metabolic syndrome at the time of endometrial cancer diagnosis, rather than 325 of 520 participants in the primary analysis. Use of the medication alternative metabolic syndrome criteria instead of the primary harmonized criteria changed six study participants' metabolic syndrome status. However, since all six participants were alive and had not experienced cancer recurrences at the end of follow-up, the number of events that occurred by metabolic syndrome status was the same for both metabolic syndrome definitions. For example, regardless of which 
metabolic syndrome criteria were used, 77 and 29 overall deaths occurred among endometrial cancer survivors with and without metabolic syndrome, respectively.

As mentioned above (Section 4.1.4.), harmonized metabolic syndrome was associated with worse overall survival but not disease-free survival, endometrial cancer-specific survival or the time to endometrial cancer recurrence. In comparison, no associations between the medication alternative metabolic syndrome criteria and survival outcomes were observed in multivariable-adjusted analyses (Table 5).

Table 5. Disease-Free Survival, Overall Survival, Endometrial Cancer-Specific Survival and Recurrence Outcomes for Endometrial Cancer Survivors with the Medication Alternate Metabolic Syndrome Definition in the Alberta Endometrial Cancer Cohort, Alberta, Canada $(\mathrm{N}=521)$

\begin{tabular}{|c|c|c|c|c|}
\hline & $\begin{array}{l}\text { Disease-Free Survival } \\
\qquad(\mathrm{n}=125 / 521)\end{array}$ & $\begin{array}{l}\text { Overall Survival } \\
(\mathrm{n}=106 / 521)\end{array}$ & $\begin{array}{c}\text { Endometrial Cancer- } \\
\text { Specific Survival } \\
(n=48 / 521)\end{array}$ & $\begin{array}{l}\text { Recurrence } \\
(n=69 / 521)\end{array}$ \\
\hline & $\begin{array}{l}\text { Events Multivariable } \\
\text { /Cases HR }(95 \% \mathrm{CI})\end{array}$ & $\begin{array}{l}\text { Events Multivariable } \\
\text { /Cases HR }(95 \% \mathrm{CI})\end{array}$ & $\begin{array}{l}\text { Events Multivariable } \\
\text { /Cases HR }(95 \% \mathrm{CI})\end{array}$ & $\begin{array}{l}\text { Events Multivariable } \\
\text { /Cases HR }(95 \% \mathrm{CI})\end{array}$ \\
\hline \multicolumn{5}{|c|}{ Alternative Metabolic Syndrome } \\
\hline No & $38 / 1911.00$ & $29 / 1911.00$ & $11 / 1911.00$ & $22 / 1911.00$ \\
\hline Yes & $87 / 3300.98(0.63-1.52)$ & $77 / 3301.35(0.83-2.20)$ & $37 / 3301.75(0.72-4.25)$ & $47 / 3300.74(0.41-1.33)$ \\
\hline
\end{tabular}

${ }^{a}$ Hazard Ratios (HR), Confidence Interval (CI).

${ }^{b}$ Multivariable models adjusted for stage (I/missing, II, III/IV), grade (I/II, III, missing), primary treatment(s) (hysterectomy, hysterectomy/chemotherapy, hysterectomy/radiation, hysterectomy/chemotherapy/ radiation and/or hormone therapy, missing treatment), baseline age (years), non-linear age $\left(\mathrm{age}^{2}\right)$ and continuous BMI $\left(\mathrm{kg} / \mathrm{m}^{2}\right)$. Disease-free survival model additionally adjusted for time to first new primary cancer and number of major comorbidities (zero, one, $\geq$ two). Overall survival additionally adjusted for time to first new primary cancer/recurrence and number of major comorbidities (zero, one, $\geq$ two). Endometrial cancer-specific survival additionally adjusted for time to first recurrence.

\subsubsection{Five- and Ten-Year Restricted Analyses}

Provided that the models assessing the number of metabolic syndrome components with disease-free, overall and endometrial cancer-specific survival did not satisfy the proportional hazards assumption in the primary analyses (Section 4.1.4.), they were further assessed in exploratory five- and ten-year restricted analyses. The association between ten-year endometrial 
cancer-specific survival and the number of metabolic syndrome components did not meet the proportional hazards assumption and was therefore not reported (Table 6). There was insufficient evidence of an association between the number of metabolic syndrome components with five-year disease-free survival, overall survival or endometrial cancer-specific survival in multivariable analyses. In the ten-year multivariable analysis, null associations were noted for the relationship between the number of metabolic syndrome components and disease-free survival, whereas participants with four or five metabolic syndrome components had increased ten-year overall survival compared to those with zero or one components (Table 6).

Table 6. Five- and Ten-Year Disease-Free Survival, Overall Survival and Endometrial Cancer-Specific Survival Outcomes for Endometrial Cancer Survivors by Number of Metabolic Syndrome Components in the Alberta Endometrial Cancer Cohort, Alberta, Canada ( $\mathrm{N}=520)$

\begin{tabular}{|c|c|c|c|c|c|c|}
\hline & \multicolumn{2}{|c|}{ Disease-Free Survival } & \multicolumn{2}{|c|}{ Overall Survival } & \multicolumn{2}{|c|}{$\begin{array}{c}\text { Endometrial Cancer- } \\
\text { Specific Survival }\end{array}$} \\
\hline & $\begin{array}{l}\text { Events/ } \\
\text { Cases } \\
\end{array}$ & $\begin{array}{l}\text { Multivariable HR } \\
(95 \% \mathrm{CI})\end{array}$ & $\begin{array}{l}\text { Events/ } \\
\text { Cases } \\
\end{array}$ & $\begin{array}{l}\text { Multivariable HR } \\
(95 \% \mathrm{CI})\end{array}$ & $\begin{array}{l}\text { Events/ } \\
\text { Cases } \\
\end{array}$ & $\begin{array}{l}\text { Multivariable HR } \\
(95 \% \text { CI })\end{array}$ \\
\hline \multicolumn{7}{|c|}{ Five Years } \\
\hline \multicolumn{7}{|c|}{ Metabolic Syndrome Components } \\
\hline $0-1$ & $8 / 81$ & 1.00 & $3 / 81$ & 1.00 & $2 / 81$ & 1.00 \\
\hline $2-3$ & $28 / 261$ & $0.55(0.23-1.32)$ & $20 / 261$ & $1.10(0.30-4.00)$ & $16 / 261$ & $1.48(0.43-5.13)$ \\
\hline $4-5$ & $28 / 178$ & $0.64(0.25-1.65)$ & $17 / 178$ & $0.74(0.18-3.03)$ & $15 / 178$ & $1.26(0.28-5.70)$ \\
\hline $\mathrm{P}$ for Trend & & 0.69 & & 0.41 & & 0.99 \\
\hline \multicolumn{7}{|c|}{ Ten Years } \\
\hline \multicolumn{7}{|c|}{ Metabolic Syndrome Components } \\
\hline $0-1$ & $12 / 81$ & 1.00 & $11 / 81$ & 1.00 & $5 / 81$ & 1.00 \\
\hline $2-3$ & $44 / 261$ & $0.71(0.35-1.42)$ & $34 / 261$ & $0.63(0.30-1.34)$ & $23 / 261$ & NR \\
\hline $4-5$ & $33 / 178$ & $0.62(0.29-1.32)$ & $23 / 178$ & $0.40(0.17-0.95)$ & $17 / 178$ & NR \\
\hline $\mathrm{P}$ for Trend & & 0.25 & & 0.03 & & NR \\
\hline
\end{tabular}

${ }^{a}$ Hazard Ratios (HR), Confidence Interval (CI), Not Reported (NR).

${ }^{b}$ Multivariable models adjusted for stage (I/missing, II, III/IV), grade (I/II, III, missing), primary treatment(s) (hysterectomy, hysterectomy/chemotherapy, hysterectomy/radiation, hysterectomy/chemotherapy/ radiation and/or hormone therapy, missing treatment), baseline age (years), non-linear age (age ${ }^{2}$ ) and continuous BMI $\left(\mathrm{kg} / \mathrm{m}^{2}\right)$. Disease-free survival model additionally adjusted for time to first new primary cancer. Overall survival additionally adjusted for time to first new primary cancer/recurrence. Endometrial cancer-specific survival additionally adjusted for time to first recurrence. 
Additionally, the proportional hazards assumption was not satisfied in the primary models for fasting blood glucose $(\geq 100 \mathrm{mg} / \mathrm{dL})$ with disease-free and overall survival nor the models for continuous fasting blood glucose (per five $\mathrm{mg} / \mathrm{dL}$ increase) with endometrial cancerspecific survival. Fasting blood glucose $(\geq 100 \mathrm{mg} / \mathrm{dL})$ was associated with improved five-year $\left(\mathrm{HR}_{5}\right.$ year $\left.=0.51,95 \% \mathrm{CI}=0.29-0.90\right)$ and ten-year disease-free survival $\left(\mathrm{HR}_{10}\right.$ year $=0.51,95 \%$ $\mathrm{CI}=0.32-0.80)$. Participants with elevated fasting blood glucose levels $(\geq 100 \mathrm{mg} / \mathrm{dL})$ also had statistically significantly better ten-year $\left(\mathrm{HR}_{10}\right.$ year $\left.=0.49,95 \% \mathrm{CI}=0.29-0.84\right)$, but not five-year, overall survival $\left(\mathrm{HR}_{5}\right.$ year $\left.=0.57,95 \% \mathrm{CI}=0.28-1.14\right)$ compared to those with fasting blood glucose $<100 \mathrm{mg} / \mathrm{dL}$. Moreover, linear dose-response relationships were observed per five mg/dL increase in fasting blood glucose with improved five-year $\left(\mathrm{HR}_{\mathrm{per}} 5 \mathrm{mg} / \mathrm{dL}=0.93,95 \% \mathrm{CI}=0.88-0.97\right)$ and ten-year endometrial cancer-specific survival $\left(\mathrm{HR}_{\mathrm{per}} 5 \mathrm{mg} / \mathrm{dL}=0.94,95 \% \mathrm{CI}=0.90-0.98\right)$ in the multivariable-adjusted models.

\subsection{Results Summary}

Overall, $325(60.2 \%)$ participants had metabolic syndrome defined by the harmonized criteria at the time of endometrial cancer diagnosis. During the median 14.2 years of follow-up, there were 132 recurrence and/or death events including 73 recurrences, 50 endometrial cancerspecific deaths and 111 overall deaths. Metabolic syndrome was associated with worse overall survival but not disease-free survival, endometrial cancer-specific survival or the time to recurrence when assessed with the harmonized criteria. No associations were observed between the alternate metabolic syndrome criteria and any of the outcomes. Conversely, increased number of metabolic syndrome components was associated with improved ten-year overall survival, but not any five-year survival outcomes or ten-year disease-free survival. 
Waist circumference was the only individual component of metabolic syndrome associated with worse overall survival ( $\geq 88 \mathrm{~cm}$, per five $\mathrm{cm}$ ) and disease-free survival (per five $\mathrm{cm})$ in the main multivariable-adjusted analyses. Although elevated fasting blood glucose $(\geq 100$ $\mathrm{mg} / \mathrm{dL}$ ) was associated with improved five-year disease-free survival as well as ten-year diseasefree and overall survival. Increasing fasting blood glucose (per five $\mathrm{mg} / \mathrm{dL}$ ) was also associated with better five-year and ten-year endometrial cancer-specific survival.

Lifetime recreational physical activity prior to diagnosis statistically significantly modified associations between metabolic syndrome (harmonized) and its components with overall survival and disease-free survival. Specifically, metabolic syndrome, increasing waist circumference (per five $\mathrm{cm}$ ), and hypertension were associated with worse overall survival, whereas greater HDL cholesterol levels (per five $\mathrm{mg} / \mathrm{dL}$ ) was related to improved overall and disease-free survival among endometrial cancer survivors with lower lifetime recreational physical activity (<9.7 MET-hours/week/year). Among survivors with higher lifetime recreational physical activity ( $\geq 9.7$ MET-hours/week/year), only increasing waist circumference (per five $\mathrm{cm}$ ) was associated with worse overall survival. 


\section{CHAPTER FIVE: DISCUSSION}

\subsection{Overview}

The aim of this thesis was to investigate the associations between metabolic syndrome and its components with recurrence and survival outcomes among endometrial cancer survivors. To achieve this aim, an analysis of the Alberta Endometrial Cancer Cohort $(n=540)$ that had data collected shortly after cancer diagnosis on direct measures of anthropometry, biomarkers and self-reported data on important covariates was conducted. The primary analyses of interest in this thesis were the associations between metabolic syndrome (using the harmonized criteria) with disease-free survival, overall survival, endometrial cancer-specific survival and time to recurrence. These associations were also assessed with a medication alternative definition of metabolic syndrome that included alternate fasting glucose and triglyceride medication use indicators. In the second set of analyses, the relationships between the number of metabolic syndrome components as well as each individual metabolic syndrome component with these cancer outcomes were examined. The last set of analyses assessed the associations between metabolic syndrome and its components with disease-free and overall survival by participants' lifetime recreational physical activity level.

\subsection{Previous Research on Metabolic Syndrome and Endometrial Cancer Survival}

\subsubsection{Metabolic Syndrome}

In this analysis, an approximate twofold decrease in overall survival was observed for endometrial cancer survivors with metabolic syndrome defined by the harmonized criteria compared to those without this syndrome. It was also noted that metabolic syndrome may be 
associated with worse endometrial cancer-specific survival, although this association did not reach statistical significance in the multivariable-adjusted model.

Limited research has been conducted on the associations between metabolic syndrome and survival outcomes among endometrial cancer survivors. Four previous observational epidemiologic studies have examined the relationship between metabolic syndrome and either overall or cancer-specific survival in this population. ${ }^{38,55-57}$ All of these studies were retrospective reviews of cohorts, large databases or medical records. ${ }^{38,55-57}$ To date, no standardized definition of metabolic syndrome has been adopted in this research area. This lack of standardization may be partially explained by the fact that previous studies have often relied on the presence of self-reported comorbid conditions (e.g. diabetes, hypertension, high cholesterol levels and/or high BMI levels) as alternative proxy indicators for metabolic syndrome components. The predominate use of self-reported comorbidities suggests that data on metabolic syndrome components assessed using standardized methods (i.e. anthropometric measures and fasting blood samples) may not be routinely available in these types of retrospective studies.

Two studies conducted by the Department of Gynecologic Oncology at the Zhejiang Cancer Hospital in China observed reduced overall survival among endometrial cancer survivors with the study-specific metabolic syndrome definition (presence of $\geq$ three of the following components: history of Type 2 diabetes, medically-treated hypertension, diagnosed or treated hyperlipidemia and either waist circumference $\geq 80 \mathrm{~cm}$ or BMI $\geq 28 \mathrm{~kg} / \mathrm{m}^{2}$ ), relative to those without this syndrome. ${ }^{56,57}$ Although these studies reported statistically significant results, these findings should be interpreted with caution since the analyses included limited adjustment for known prognostic factors (e.g. participant age, cancer treatment) and the resulting estimates had low precision. While previous research, as well as the current thesis, have consistently reported 
poor overall survival among endometrial cancer survivors with metabolic syndrome, additional studies are needed to contribute to this under-studied research topic.

Two prior studies ${ }^{38,55}$ reported statistically significant associations between metabolic syndrome and endometrial cancer-specific survival. Jin and colleagues ${ }^{55}$ included 10,090 endometrial cancer survivors from the SEER-Medicare database, of whom 1,786 later had a cancer-specific death. Medicare claims for metabolic syndrome were associated with worse endometrial cancer-specific survival for stage I/II ( $\mathrm{HR}=1.28,95 \% \mathrm{CI}=1.09-1.53)$, but not stage $\mathrm{III} / \mathrm{IVa}(\mathrm{HR}=1.18,95 \% \mathrm{CI}=0.93-1.49)$ endometrial cancer survivors. Similarly, Bjorge et al. ${ }^{38}$ reported that metabolic syndrome was associated with 1.56 (95\% CI=1.32-1.84) times the hazard of uterine cancer-specific mortality per one-unit increment of composite metabolic syndrome Zscore. The Me-Can project identified women with endometrial cancer based on death records; 129 cancer-specific deaths were included in the project, although only 58\% of these study participants had a prior diagnosis of incident endometrial cancer in the cohort. ${ }^{38}$ Moreover, tumour and treatment characteristics were unknown, and therefore not included in the statistical models. ${ }^{38}$ In contrast to prior research, the relationship between metabolic syndrome and endometrial cancer-specific survival did not reach statistical significance in this thesis, although this lack of a statistically significant association is likely attributable to the limited number of endometrial cancer specific-events that occurred in the Alberta Endometrial Cancer Cohort.

No known study has assessed the association between metabolic syndrome and recurrence outcomes among endometrial cancer survivors. In the current thesis, there was insufficient evidence of an association between metabolic syndrome and disease-free survival or the time to endometrial cancer recurrence in multivariable-adjusted models. This lack of association might be attributable to the small numbers of recurrences that occurred in the Alberta 
Endometrial Cancer Cohort. However, given that the relationship between metabolic syndrome and recurrence may act as an early indicator of poor survival for endometrial cancer survivors, understanding this area of research should be the focus of future studies.

In addition to the assessment of metabolic syndrome with the primary harmonized criteria, this thesis assessed the medication alternative definition that included treatment use indicators for the fasting blood glucose and triglyceride components. Only participants using medication within one year of endometrial cancer diagnosis were included in the alternative medication definition to maintain a consistent time frame with the hypertension criteria. When the medication alternative metabolic syndrome criteria were used, no associations were observed for any of the survival outcomes. The attenuation in the estimates that incorporated the inclusion of medication use as a proxy for high glucose and triglyceride levels compared to the primary metabolic syndrome criteria may be related to the management of these risk factors as a result of taking these medications.

The relationship between the number of metabolic syndrome components and survival outcomes has not been previously investigated. In this thesis, only eleven participants had no metabolic syndrome risk factors, so the reference group included survivors with both zero and one risk factor. The relationship between the number of metabolic syndrome components (zeroone, two-three, four-five) and survival outcomes varied over time in the main analysis. Therefore, exploratory five- and ten-year restricted analyses were conducted. Contrary to the main results in which metabolic syndrome ( $\geq$ three components) was associated with poor overall survival, endometrial cancer survivors with four or five, compared to zero or one, metabolic syndrome components had better ten-year overall survival. No associations between the number of metabolic syndrome components and overall survival were seen for five-year 
outcomes. It is important to note that these analyses do not account for any potential change in the number of metabolic syndrome components during follow-up and assumes that each metabolic syndrome contributes equally to individuals' heath status.

\subsubsection{Obesity and Waist Circumference}

In this thesis, the results suggest that the association between metabolic syndrome and endometrial cancer survival was largely attributable to the association between waist circumference and survival. Compared to endometrial cancer survivors with a waist circumference $<88 \mathrm{~cm}$, survivors with a waist circumference $\geq 88 \mathrm{~cm}$ had a twofold decrease in overall survival. For every five $\mathrm{cm}$ increase in waist circumference $\geq 88 \mathrm{~cm}$, endometrial cancer survivors were estimated to have a $11 \%$ decrease in disease-free survival and a $21 \%$ decrease in overall survival. Although the associations between greater waist circumference $(\geq 88 \mathrm{~cm})$ and worse disease-free survival, endometrial cancer-specific survival and recurrence did not reach statistical significance in multivariable models, these findings may still be clinically relevant.

Both waist circumference and BMI have been previously positively correlated with estrogen levels in endometrial cancer survivors. ${ }^{14,121}$ Excess adipose tissue can produce elevated concentrations of estrogens, which in the absence of sufficient progesterone is a known risk factor of endometrial cancer tumour proliferation. ${ }^{14,121}$ The adipose tissue and unopposed estrogen mechanism of pathogenesis may partially explain the attenuation of the associations between waist circumference with disease-free survival, endometrial cancer-specific survival and recurrence in multivariable models that adjusted for BMI. Waist circumference is also a strong indicator of central adiposity which has been positively associated with other biological 
mechanisms related to tumour pathogenesis, such as hyperglycemia, dyslipidemia, hyperinsulinemia and systemic inflammation. ${ }^{14}$

This thesis is the first known project to directly assess the relationship between measured central adiposity (i.e. waist circumference) and survival outcomes among endometrial cancer survivors. In the general population, the Nurses' Health Study previously reported that for every approximate ten $\mathrm{cm}$ increase in waist circumference, women had a $34 \%(95 \% \mathrm{CI}=23-39 \%)$ increase in all-cause mortality and a 18\% (95\% CI=9-28\%) increase in obesity-related (colon cancer, breast cancer, pancreatic cancer, uterine, ovarian, and kidney cancer) cancer mortality in multivariable adjusted models which included adjustments for BMI. ${ }^{122}$

Most of the previous literature in the endometrial cancer survivor population, including a systematic review and meta-analysis of 18 cohort studies conducted by Secord et al. ${ }^{99}$ has focused on the relationship between BMI and survival outcomes. Secord et al. ${ }^{99}$ noted a doseresponse relationship between increasing BMI and reduced survival that reached statistical significance among endometrial cancer survivors with a BMI $\geq 40 \mathrm{~kg} / \mathrm{m}^{2}$. More recently, elevated BMI has been associated with reduced overall survival and endometrial cancer-specific survival in four ${ }^{5,61,64,94}$ and five studies, ${ }^{35,38,59,60,66}$ respectively. However, two studies have reported findings that Type II endometrial cancer survivors with a BMI above the normal range $\left(18.5-24.9 \mathrm{~kg} / \mathrm{m}^{2}\right)$ experience better endometrial cancer-specific survival. ${ }^{5,91}$ This improved survival has become known as the obesity paradox hypothesis, which suggests that obesity may be protective of incidence and mortality for some cancer sites. ${ }^{14}$ Yet, several other potential reasons to explain the obesity paradox in cancer patients have been proposed, including residual confounding by age, smoking and physical activity which all impact measures of BMI and survival. ${ }^{14}$ Additionally, some have suggested that the obesity paradox is related to reverse 
causation proposing that lower body weight at cancer diagnosis may be the result of cancer cachexia rather than health status. ${ }^{14}$ Moreover, the association between obesity and less aggressive tumour characteristics may partially explain these results. ${ }^{14}$

One study to date has observed a statistically significant association between BMI and endometrial cancer recurrence. ${ }^{68}$ Canlorbe et al. ${ }^{68}$ assessed the relationship between BMI and recurrence according to the ESMO risk groups and only observed an association among endometrial cancer survivors in the ESMO high-risk group. Several studies have failed to observe a statistically significant relationship between BMI with overall survival, ${ }^{41,65,67,70,74,76,78-}$ $81,83,84,87,88$ endometrial cancer-specific survival ${ }^{42,55,64,65,75,84,85,88,89}$ and recurrence. ${ }^{63,67,69,72,74-}$ 76,78,82-84,86,90,92 Study design and methodical approaches may contribute to some of the null results reported. Of note, many of these estimates were derived from studies with small samples (n <250), low numbers of events and univariate analyses which may have contributed to the null findings reported by some of these studies. ${ }^{64,70,74,75,77,80,87}$

Although the body of literature has largely focused on the associations between BMI and prognostic outcomes, the current findings are generally consistent with prior research that observed worse overall survival with increasing obesity among endometrial cancer survivors. The current findings also provide some indication that obesity, particularly central adiposity, may be a more relevant risk factor for overall survival than endometrial cancer-specific survival and recurrence outcomes.

\subsubsection{Diabetes}

There was no evidence of a relationship between fasting blood glucose levels and any of the survival outcomes in the current thesis' primary analysis. Additionally, the proportional 
hazards assumption was not satisfied in the main disease-free or overall survival models for fasting blood glucose ( $\geq 100 \mathrm{mg} / \mathrm{dL}$ ), nor the endometrial cancer-specific survival models for continuous fasting blood glucose (per five $\mathrm{mg} / \mathrm{dL}$ increase). In the five- and ten-year restricted analyses, blood glucose ( $\geq 100 \mathrm{mg} / \mathrm{dL})$ was associated with improved five-year disease-free survival and ten-year disease-free and overall survival. A positive dose-response relationship was also noted for improved five- and ten-year endometrial cancer-specific survival per five $\mathrm{mg} / \mathrm{dL}$ increase in fasting blood glucose.

Three previous studies have assessed the association between pre-diagnosis blood glucose levels and either overall survival ${ }^{70,80}$ or endometrial cancer-specific survival, ${ }^{38}$ reporting no statistically significant associations among endometrial cancer survivors. Most of the studies that have explored the relationship between diabetes and overall survival have reported a harmful relationship, ${ }^{5,40-42,78,79,88,95,101,102,105}$ whereas only half of the studies observed worse endometrial cancer-specific survival among endometrial cancer survivors with diabetes. ${ }^{5,79,91,95,103-105,107,108}$ Improved three-year survival was noted in one study ${ }^{106}$ for survivors with diabetes compared to those without diabetes. Of the studies that assessed the relationship between diabetes and recurrence outcomes, ${ }^{75,76,78,84,90,100}$ only Ko et al. ${ }^{78}$ noted a statistically significant relationship with reduced disease-free survival. Generally, this literature has been limited by the number of participants with diabetes and the number of events that occurred in each study. ${ }_{4} 2,75,77,88$

The results from the primary analyses in this thesis align with the null findings previously reported for the association between fasting blood glucose and prognostic outcomes among endometrial cancer survivors. However, the exploratory five- and ten-year restricted analyses in this study suggested a protective association with survival outcomes. 
The discrepancies between these different study findings may be partially explained by different criteria used to define diabetes and hyperglycemia in the metabolic syndrome. Compared to the hyperglycemia criteria of metabolic syndrome, which defines fasting blood glucose as $\geq 100 \mathrm{mg} / \mathrm{dL}$, diabetes is generally diagnosed at fasting glucose levels of 126 $\mathrm{mg} / \mathrm{dL} \cdot{ }^{46,125}$ Additionally, diabetes is characterized by impaired insulin production or reduced cellular response to insulin. ${ }^{125}$ Insulin resistance has been linked to accelerated transformation of androstenedione into estrogen, thus increasing the levels of circulating unopposed estrogen. ${ }^{126}$ Additionally, hyperinsulinemia has been associated with greater invasion of endometrial cancer cells, inhibition of apoptosis of cancer cells, promotion of angiogenesis in tumours and lymph node infiltration, independently of glucose concentrations. ${ }^{126}$ While hyperglycemia has been linked to metabolic reprogramming of tumour cells, tumour inflammation, angiogenesis and inhibition of macrophages function in endometrial cancer survivors, studies conducted in mice models failed to demonstrate a positive association between glucose levels and tumour growth in the absence of hyperinsulinemia. ${ }^{14,126}$ Therefore, this difference in the definition criteria used for hyperglycemia as part of metabolic syndrome and for the diagnosis of diabetes may explain these discrepancies in study findings.

\subsubsection{Hypertension}

Self-reported hypertension was not associated with any of the prognostic outcomes in this thesis. The literature regarding the association between hypertension and survival among women living with endometrial cancer remains mixed. Only Bjorge et al. ${ }^{38}$ have examined the association between measured blood pressure and survival outcomes. The authors reported some evidence of a linear trend for increasing quintile of DBP (p-trend <0.01) with decreased survival, 
but no relationship between continuous blood pressure, quintile of SBP or DBP with endometrial cancer-specific survival. ${ }^{38}$

Worse overall survival has been noted for endometrial cancer survivors with hypertension compared to those without. ${ }^{41,101}$ Decreased overall survival with hypertension has been particularly evident among Type II endometrial cancer survivors. ${ }^{43,94}$ However, the majority of this literature has failed to observe either crude ${ }^{80,111}$ or adjusted $^{40,63,70,88,95,110}$ associations between hypertension and overall survival in this population. There has been one study ${ }^{88}$ that reported improved overall survival with hypertension among Black endometrial cancer survivors. Of the seven studies that assessed the association between hypertension and endometrial cancer-specific survival, no studies observed a harmful association, one study ${ }^{88}$ observed a positive association and six studies reported null associations. ${ }^{55,75,77,84,91,95}$ There is limited research ${ }^{63,75,84}$ that has investigated the association between hypertension and endometrial cancer recurrence. Two studies have previously ${ }^{75,84}$ observed null associations and one reported a protective relationship between hypertension and recurrence outcomes. ${ }^{63}$

While some literature among endometrial cancer survivors supports the notion that hypertension reduces overall survival, there is less evidence of a relationship with endometrial cancer-specific outcomes. The current results add to the body of literature reporting null associations between hypertension with recurrence and survival outcomes.

The potential mechanisms between hypertension and endometrial cancer-specific survival and recurrence are not well understood. ${ }^{126}$ However, it is plausible that the association between hypertension and overall survival among endometrial cancer survival is driven by cardiovascular disease. Felix and colleagues ${ }^{45}$ have previously reported that endometrial cancer survivors in the 
Iowa Women's Health Study with hypertension had increased cardiovascular disease mortality compared to normotensive survivors.

\subsubsection{Triglycerides and HDL Cholesterol}

In this thesis, blood triglycerides and HDL cholesterol were assessed via fasting blood draws at the time of endometrial cancer diagnosis. Based on the metabolic syndrome threshold of $150 \mathrm{mg} / \mathrm{dL}$, blood triglycerides levels were not associated with any prognostic outcomes, nor was there evidence of a linear trend. Nearly all participants $(n=440,81.5 \%)$ had blood HDL cholesterol concentrations $<50 \mathrm{mg} / \mathrm{dL}$. Therefore, the protective relationship observed between low HDL cholesterol and improved endometrial-cancer survival is likely an artifact of the data rather than a clinically meaningful result. Moreover, for every additional five $\mathrm{mg} / \mathrm{dL}$ increase in HDL cholesterol $\geq 50 \mathrm{mg} / \mathrm{dL}$, endometrial cancer survivors were estimated to have improved overall and disease-free survival, although these relationships did not reach statistical significance. The linear trend, though also not statistically significant, supports the notion that the protective association seen with endometrial cancer-specific survival was the result of the homogeneous sample rather than biological plausibility.

Previous literature has used multiple variations of dyslipidemia and abnormal blood lipid definitions in the assessment of recurrence and survival outcomes. While the metabolic syndrome criteria specifically assesses blood triglycerides and HDL cholesterol, dyslipidemia generally refers to elevated blood triglycerides, total cholesterol, LDL and/or reduced HDL cholesterol levels. ${ }^{39}$ No statistically significant associations have been observed between triglyceride and total cholesterol levels with overall survival among endometrial cancer survivors. ${ }^{70}$ Three studies reported null associations between hypercholesterolemia, ${ }^{38,84}$ 
dyslipidemia ${ }^{55}$ as well as hypertriglyceridemia ${ }^{38,55}$ with endometrial cancer-specific survival. However, Van Arsdale et al. ${ }^{91}$ noted improved endometrial cancer-specific survival for Type II endometrial cancer survivors with hyperlipidemia, but a null association among women living with Type I endometrial cancer. The association between dyslipidemia and endometrial cancer recurrence has been assessed in one study, which failed to observe an association in the univariable analysis. $^{84}$

The null results in this thesis are therefore consistent with previous research. The potential mechanisms by which triglyceride and cholesterol impact endometrial cancer have been largely assessed in terms of cancer incidence. Less is understood about how abnormal blood lipids impact endometrial cancer progression. ${ }^{126}$ It has been suggested that elevated free fatty acids may be related to the progression of endometrial cancer via an estrogen receptor-dependent pathway. ${ }^{126}$ A proposed relationship between HDL metabolism and cellular lipid homeostasis regulation has also been suggested for gynecologic cancer pathogenesis. ${ }^{127}$

\subsubsection{Physical Activity Modification}

In this analysis, the associations between metabolic syndrome and its components with survival outcomes depended on the participants' lifetime recreational physical activity prior to diagnosis (as assessed in terms of MET-hours/week/year). For endometrial cancer survivors with lower lifetime recreational physical activity ( $<9.7$ MET-hours/week/year), those with metabolic syndrome had a 2.5 -fold decrease in overall survival compared to participants without the syndrome. Conversely, the association between metabolic syndrome and overall survival did not reach statistical significance amongst survivors with higher lifetime recreational physical activity ( $\geq 9.7$ MET-hours/week/year). A harmful relationship between hypertension and overall 
survival, as well as protective associations with HDL cholesterol, and overall and disease-free survival were only observed among endometrial cancer survivors with lower recreational physical activity levels. Finally, increasing waist circumference was associated with worse overall survival for both women with lower as well as higher lifetime recreational physical activity, although the association was attenuated among endometrial cancer survivors with greater lifetime recreational physical activity compared to those with lower lifetime physical activity.

No known prior study has examined metabolic syndrome or its components with survival outcomes among endometrial cancer survivors in the context of physical activity. Generally, these findings add to a larger body of literature that suggests cancer survivors with greater physical activity participation experience better survival. ${ }^{19}$ Overall cancer survivors with the highest compared to lowest levels of pre-diagnosis physical activity have been reported to have an $18 \%(95 \% \mathrm{CI}=14-21 \%)$ improvement in total cancer-specific survival. ${ }^{19}$ However, women living with female reproductive cancers (cervical, endometrial, and ovarian cancers) with the highest versus lowest pre-diagnoses physical activity did not experience a statistically significant difference in overall or cancer-specific survival. ${ }^{19}$ Specific survival estimates with physical activity among endometrial cancer survivors are limited. ${ }^{19,123}$ In the NIH-AARP Diet and Health Study, Arem and colleagues ${ }^{60}$ previously reported endometrial cancer survivors with higher prediagnosis recreational moderate-to-vigorous-intensity physical activity had better five-year overall survival, but not ten-year overall survival or endometrial specific-survival. The authors also investigated the association between pre-diagnosis physical activity and survival among endometrial cancer survivors in the Women's Health Initiative, although no associations were observed with overall and endometrial cancer-specific survival outcomes. ${ }^{59}$ 
Our findings suggest that endometrial cancer survivors with greater lifetime recreational physical activity participation do not experience the same harmful associations between metabolic syndrome, waist circumference, and hypertension with overall survival compared to those with lower amounts of lifetime physical activity participation. The overall survival benefits experienced with greater physical activity is thought to relate to lower levels of obesity and improved cardiorespiratory fitness, therefore reducing cardiac morbidity and mortality. ${ }^{128,129}$ Moreover, physical activity has been reported to improve insulin sensitivity and reduce systemic inflammation, blood pressure and visceral adiposity in the general population. ${ }^{130}$ It is therefore possible that metabolic syndrome and the accompanying pathophysiology (e.g. chronic inflammation) may be less severe in women who regularly participate in physical activity.

\subsection{Strengths of Project}

The substantial study follow-up time and well-defined study population are key strengths of the current project. Participants were originally identified through the Alberta Cancer Registry, which has a $\geq 95 \%$ case ascertainment rate ${ }^{114}$ of histologically confirmed, endometrial cancer cases and are therefore representative of the larger endometrial cancer survivor population living in Alberta. The median follow-up time was 14.2 years (range: $0.3-16.5$ years) which provides an appropriate duration of time for events to occur in this population. ${ }^{131}$ Moreover, outcome ascertainment was conducted through data linkage with national and provincial registries which assures that the survival data are comprehensive. Metabolic syndrome exposure data including direct anthropometric measures and blood assays were taken in multiples at the time of endometrial cancer diagnosis and provide valid and reliable data with a clear temporal relationship to the outcomes assessed. Interviewers trained with cognitive interview methods 
administered questionnaires at the time of endometrial cancer diagnosis to collect data on potential covariates. Cognitive interviewing data collection methods have been shown to improve participants' long-term recall ability and have been successfully used in a variety of surveys. ${ }^{117}$

\subsection{Limitations}

The limitations of this study also need to be considered when assessing these results. The limitations include threats to internal and external validity such as selection bias, misclassification bias, type I and II error, confounding and generalizability.

\subsubsection{Internal Validity}

Internal validity is the extent to which the observed associations represent the true relationship of interest. The internal validity of a study can be threatened by either random or systematic error. As the name suggests, random error occurs by chance and threatens all studies, although has a greater impact on studies with smaller sample sizes. Systematic error, on the other hand, is caused by defects in the study approach or methods which impact the accuracy of the estimates. Selection bias, measurement bias and confounding are the most common types of systematic error. ${ }^{132}$

\subsubsection{Selection Bias}

Selection bias results from study design defects and other factors that impact who is included in a study. The current project was composed of endometrial cancer cases from the original population-based case-control study, therefore the inclusion in the current study was 
contingent upon participation in the prior study. Of the eligible endometrial cancer cases identified between 2002 and 2006 by the Alberta Cancer Registry, 61\% participated in the casecontrol study. Given that these endometrial cancer survivors self-selected to participate in the project, they represent women who were first and foremost healthy enough to participate and secondly potentially more health conscious than women who refused to participate.

Of the 540 participants who were eligible for this analysis, 27 participants were missing $\geq$ one metabolic syndrome component. Seven of these endometrial cancer survivors still met $\geq$ three metabolic syndrome components criteria and were classified as having metabolic syndrome. Participants missing at least one metabolic syndrome component were not included in the component models and survivors who could not be categorized by metabolic syndrome status were missing from the metabolic syndrome analysis. Incomplete fasting blood assays was the primary reason for partial metabolic syndrome data, and likely occurred at random. Moreover, endometrial cancer survivors included in the component analyses $(n=513)$ were similar in terms of mean age at diagnosis, FIGO grade and BMI to those with incomplete metabolic syndrome data $(\mathrm{n}=27)($ Age $n=513: 59.1 \pm 9.4$ years, $n=27: 57.8 \pm 8.3$ years; Cancer grade $n=513:$ I/II $=$ $76.2 \%, \mathrm{III}=13.4 \%, \mathrm{NR}=10.3 \%, \mathrm{n}=27: \mathrm{I} / \mathrm{II}=81.5 \%, \mathrm{III}=14.8 \%, \mathrm{NR}=3.7 \% ; \mathrm{BMI} \mathrm{n}=513: 32.1$ $\left.\pm 7.9 \mathrm{~kg} / \mathrm{m}^{2}, \mathrm{n}=27: 35.8 \pm 6.9 \mathrm{~kg} / \mathrm{m}^{2}\right)$. Although, endometrial cancer survivors included in the component analyses had slightly fewer stage II tumours $(\mathrm{n}=513: \mathrm{I}=80.1 \%, \mathrm{II}=11.9 \%$, III/IV = $8.0 \%, \mathrm{n}=27: \mathrm{I}=63.0 \%, \mathrm{II}=29.6 \%, \mathrm{III} / \mathrm{IV}=7.4 \%)$ and comorbidities $(\mathrm{n}=513:$ zero $=88.1 \%$, one $=9.3 \%, \geq$ two $=2.5 \%, \mathrm{n}=27:$ zero $=81.5 \%$, one $=18.5 \%, \geq$ two $=0 \%)$ than the survivors missing metabolic syndrome data. 


\subsubsection{Misclassification Bias}

Misclassification bias results from systematic error in the measurement of the exposure or outcome. The primary aim of the original population case-control study was to address the association between physical activity and endometrial cancer risk, while the association with metabolic syndrome was a later addition to this research program. Given that the original funding and participants' measurements were obtained in the case-control study prior to the addition of metabolic syndrome, not all metabolic syndrome components were fully captured. While the majority of the metabolic syndrome components were available from direct measurements (i.e. waist circumference, fasting blood glucose, triglycerides, HDL cholesterol), blood pressure was not assessed. Data on self-reported hypertension diagnosis and/or antihypertensive mediation use were proxy measures in the metabolic syndrome criteria, meaning that participants with undiagnosed hypertension would have been misclassified as normotensive. Endometrial cancer survivors have been reported to have more diagnosed and undiagnosed hypertension compared to women in the general population. ${ }^{6}$ Since all participants were equally likely to have undiagnosed hypertension, this misclassification is non-differential in nature. Therefore, underdiagnosed and unreported hypertension in the current project may have attenuated the estimates of associations with hypertension and potentially metabolic syndrome.

Moreover, the artificial categorization of metabolic syndrome components and subsequent classification of metabolic syndrome relies heavily on threshold values which can result in the loss of information. This categorization could have contributed to the unexpected protective associations observed in the HDL cholesterol and fasting blood glucose analyses. For instance, participants with fasting blood glucose levels of $101 \mathrm{mg} / \mathrm{dL}$ and $126 \mathrm{mg} / \mathrm{dL}$ were both categorized as meeting the blood glucose criteria despite the fact that participants with blood 
glucose levels of $126 \mathrm{mg} / \mathrm{dL}$ would be considered diabetic while participants with fasting blood glucose levels of $101 \mathrm{mg} / \mathrm{dL}$ have a nearly normal fasting blood glucose concentration. One possible explanation for the protective association observed in the five- and ten-year restricted glucose analyses is that the threshold of $100 \mathrm{mg} / \mathrm{dL}$ was too low as a cut-off point to represent two meaningfully distinct groups in these analyses. Therefore, the comparison and reference groups would be more similar than anticipated. However, the practice of categorization has long been accepted in the health field to facilitate real-world decisions and treatments, and the use of these cut-off points have been previously validated as being associated with increased disease risk. ${ }^{132-135}$

Metabolic syndrome was only assessed at the time of cancer diagnosis and may not represent participants' health status for the entire follow-up period. Previous findings from the Atherosclerosis Risk in Communities Study suggest that most individuals (76\%) experienced increased severity of metabolic syndrome over time and medical treatment of the individual risk factors was ineffective at reducing the severity. ${ }^{124}$ Therefore, the burden of metabolic syndrome among endometrial cancer survivors in the current study may have been underestimated since participants who did not have metabolic syndrome at baseline may have developed it during the follow-up period. This change over time would lead to the exposed and non-exposed groups being more similar and the estimates would be skewed towards the null.

\subsubsection{Power, Precision, and Residual Confounding}

Statistical power is the probability of detecting an association when that relationship truly exists. If a study is underpowered, the probability of failing to observe an association when in 
truth the relationship exists, committing a type II error, increases. Statistical power is impacted by the study's sample size, magnitude of effect and the level of statistical significance that is set. Increasing sample size is the most common method used to improve statistical power and will increase the precision of the estimates as reflected in the width of the confidence intervals. Statistical significance was set at five percent in the current thesis, therefore the probability of observing a statistically significant association by chance, type I error, was five percent. As the number of statistical tests performed increases, so does the chance that the observed results are the product of type I error. ${ }^{132}$

Due to the small sample size and limited number of events, the current study was likely underpowered to observe statistically significant associations and therefore at risk of type II error. This risk of type II error is particularly relevant for the endometrial cancer-specific survival and recurrence results, which should be interpreted with caution because of the small number of events in the categorical analyses. The lower precision of the estimates reflected in the wide confidence intervals are indicative of underpowered analyses. Specifically, the association between HDL cholesterol and endometrial-cancer specific survival is likely an erroneous result and an artifact of the limited number of events in the reference group.

Many associations were assessed in this thesis because of the number of metabolic syndrome components and number of outcomes considered. While it is possible that some associations were the result of type I error, the consistency across outcomes and analyses would suggest that the results were not as a result of chance. Moreover, a basic biological plausibility model was determined a priori and included in all analyses to reduce the number of analyses preformed. 
Confounding is the intermixing of an additional factor in the association of interest. Confounding variables are characterized as independent risk factors for the outcome, associated with the exposure and not on the causal pathway between exposure and outcome. ${ }^{132}$ Possible confounding factors were assessed using backwards elimination and based on a priori biological plausibility and existing literature. This method is considered an effective way of assessing potential confounding factors. However, it is possible that unmeasured factors may have resulted in residual confounding in the present analyses.

\subsubsection{External Validity}

The extent to which the results of one study can be generalized beyond its original context to the larger population represents a study's external validity. This type of validity is contingent upon the study first being deemed internally valid. Results from studies with samples defined by rigorous inclusion criteria may not be generalizable to the broader population. In the current thesis, there were no restrictions on demographic characteristic except age, in which all women between 30 and 80 years of age were eligible. Provided that the vast majority of women at risk of developing endometrial cancer are within this age range, this eligibility criteria is not likely a limiting factor. ${ }^{3}$ Although not inclusion criteria, the study participants represent a predominantly White (93.9\%) and well educated $(67.2 \% \geq$ high school education) group of endometrial cancer survivors living in Alberta. Due to the homogenous study population, the potential impact of race and ethnic background could not be assessed in this thesis. Future research should address this gap by including racially divers endometrial cancer survivors in the assessment of metabolic syndrome with survival outcomes. Therefore, the current findings may be most applicable to White endometrial cancer survivors who have access to similar cancer 
treatments as the survivors in the Alberta Endometrial Cancer Cohort did. However, given the similarity between endometrial cancer and other obesity-linked cancers, these results may be relevant to the broader cancer survivor population.

\subsection{Future Directions}

This thesis, along with the available literature, suggest that endometrial cancer survivors with metabolic syndrome experience both reduced endometrial cancer-specific survival and overall survival. ${ }^{38,55-57}$ However, the key factors of the associations between metabolic syndrome and prognostic outcomes in endometrial cancer survivors are still debated. While this thesis and most previous research ${ }^{38,39}$ suggests that the association is primarily related to obesity and central adiposity, not all studies support this hypothesized biological link. ${ }^{55}$ Additional research is needed to understand the biological mechanism associated with metabolic syndrome and survival outcomes. Moreover, studies to date have been conducted with the assumption that all metabolic syndrome components contribute equally to endometrial cancer survival. Future studies should aim to determine the critical risk factors within the metabolic syndrome and assess the assumption that all metabolic syndrome components contribute equally to cancer prognosis. Furthermore, future research should aim to assess metabolic syndrome at multiple time points during study follow-up with objective measurements.

Provided that endometrial cancer survivors have greater amounts of cardiometabolic risk factors that may or may not be diagnosed or treated compared to the general population, ${ }^{6}$ and that endometrial cancer mortality rates are currently rising, ${ }^{11}$ identifying opportunities to intervene in this population is becoming increasingly important. This thesis provides some evidence that the associations with metabolic syndrome and its components were attenuated 
among endometrial cancer survivors with greater lifetime recreational physical activity compared to those with lower physical activity levels before cancer diagnosis. Lifestyle interventions that target weight management, healthy diets and physical activity have been proposed as means to address the growing burden of chronic conditions among endometrial cancer survivors and improve survival outcomes. ${ }^{6,51}$ The impact of lifestyle interventions on recurrence and survival outcomes among gynecological cancer survivors remains unclear. ${ }^{136}$ Moreover, it has been suggested that cancer diagnosis represents a time in which individuals are more receptive to the adoption of lifestyle changes. ${ }^{137}$ Results from the Survivors of Uterine CanCer Empowered by Exercise and Healthy Diet (SUCCEED) trial suggest that after a six-month lifestyle intervention, endometrial cancer survivors were able to lose and maintain weight loss successfully at one year follow-up with diet and physical activity. ${ }^{138}$ Future survival analyses from the SUCCEED trial ${ }^{138}$ and additional studies that assess metabolic syndrome and its components are needed to improve our understanding of the role physical activity and other lifestyle factors play in the association between metabolic syndrome and endometrial cancer survivors' prognosis.

\subsection{Conclusion}

This thesis improves our understanding of the associations between metabolic syndrome and its components with cancer recurrence and survival outcomes among endometrial cancer survivors. Endometrial cancer survivors with metabolic syndrome had reduced overall survival compared to those without this syndrome. Moreover, the results suggest that the association between metabolic syndrome and endometrial cancer survival is largely explained by central adiposity. It also illustrated that endometrial cancer survivors with greater lifetime recreational physical activity before diagnosis had improved survival despite metabolic syndrome risk factors 
compared to those with lower lifetime recreational activity levels. This thesis provides some additional evidence needed to develop and assess interventions in endometrial cancer survivors targeting early indicators of cardiovascular and metabolic disease, such as metabolic syndrome, with the ultimate aim of improving endometrial cancer survival. 


\section{REFERENCES}

1. Bray F, Ferlay J, Soerjomataram I, Siegel RL, Torre LA, Jemal A. Global cancer statistics 2018: GLOBOCAN estimates of incidence and mortality worldwide for 36 cancers in 185 countries. CA Cancer J Clin. 2018;68(6):394-424.

2. Navaneelan T. Trends in the incidence and mortality of female reproductive system cancers. Statistics Canada;2015. 82-624-X.

3. Passarello K, Kurian S, Villanueva V. Endometrial Cancer: An Overview of Pathophysiology, Management, and Care. Semin Oncol Nurs. 2019;35(2):157-165.

4. Schottenfeld D, Fraumeni JF. Cancer epidemiology and prevention. 4th ed. Oxford, New York: Oxford University Press; 2018.

5. Nagle CM, Crosbie EJ, Brand A, et al. The association between diabetes, comorbidities, body mass index and all-cause and cause-specific mortality among women with endometrial cancer. Gynecol Oncol. 2018;150(1):99-105.

6. Kitson SJ, Lindsay J, Sivalingam VN, et al. The unrecognized burden of cardiovascular risk factors in women newly diagnosed with endometrial cancer: A prospective case control study. Gynecol Oncol. 2018;148(1):154-160.

7. Brenner DR, Poirier AE, Ruan Y, et al. Estimates of the current and future burden of cancer attributable to excess body weight and abdominal adiposity in Canada. Prev Med. 2019;122:49-64.

8. Meyer LA, Broaddus RR, Lu KH. Endometrial cancer and Lynch syndrome: clinical and pathologic considerations. Cancer Control. 2009;16(1):14-22.

9. World Cancer Research Fund/American Institute for Cancer Research Continuous Update Project Expert Report. Diet, nutrition, physical activity and endometrial cancer. Available at dietandcancerreport.org. 2018.

10. Brenner DR, Weir HK, Demers AA, et al. Projected estimates of cancer in Canada in 2020. CMAJ. 2020;192(9):E199-E205.

11. Canadian Cancer Statistics Advisory Committee. Canadian Cancer Statistics 2019. Toronto, ON: Canadian Cancer Society; 2019. Available at: cancer.ca/Canadian-CancerStatistics-2019-EN (accessed March 17 2020). 2019.

12. Siegel RL, Miller KD, Jemal A. Cancer statistics, 2018. CA Cancer J Clin. 2018;68(1):730 .

13. Oliver KE, Enewold LR, Zhu K, et al. Racial disparities in histopathologic characteristics of uterine cancer are present in older, not younger blacks in an equal-access environment. Gynecol Oncol. 2011;123(1):76-81. 
14. Avgerinos KI, Spyrou N, Mantzoros CS, Dalamaga M. Obesity and cancer risk: Emerging biological mechanisms and perspectives. Metabolism. 2019;92:121-135.

15. Aune D, Navarro Rosenblatt DA, Chan DS, et al. Anthropometric factors and endometrial cancer risk: a systematic review and dose-response meta-analysis of prospective studies. Ann Oncol. 2015;26(8):1635-1648.

16. O'Flanagan CH, Bowers LW, Hursting SD. A weighty problem: metabolic perturbations and the obesity-cancer link. Horm Mol Biol Clin Investig. 2015;23(2):47-57.

17. Schmid D, Behrens G, Keimling M, Jochem C, Ricci C, Leitzmann M. A systematic review and meta-analysis of physical activity and endometrial cancer risk. Eur J Epidemiol. 2015;30(5):397-412.

18. Lynch BM. Sedentary behavior and cancer: a systematic review of the literature and proposed biological mechanisms. Cancer Epidemiol Biomarkers Prev. 2010;19(11):2691-2709.

19. Friedenreich CM, Stone CR, Cheung WY, Hayes SC. Physical Activity and Mortality in Cancer Survivors: A Systematic Review and Meta-Analysis. JNCI Cancer Spectr. 2020;4(1).

20. Nagle CM, Olsen CM, Ibiebele TI, et al. Glycemic index, glycemic load and endometrial cancer risk: results from the Australian National Endometrial Cancer study and an updated systematic review and meta-analysis. Eur J Nutr. 2013;52(2):705-715.

21. Shahdadian F, Saneei P, Milajerdi A, Esmaillzadeh A. Dietary glycemic index, glycemic load, and risk of mortality from all causes and cardiovascular diseases: a systematic review and dose-response meta-analysis of prospective cohort studies. Am J Clin Nutr. 2019;110(4):921-937.

22. Lukic M, Guha N, Licaj I, et al. Coffee Drinking and the Risk of Endometrial Cancer: An Updated Meta-Analysis of Observational Studies. Nutr Cancer. 2018;70(4):513-528.

23. Zhou B, Yang L, Sun Q, et al. Cigarette smoking and the risk of endometrial cancer: a meta-analysis. Am J Med. 2008;121(6):501-508 e503.

24. Christensen CH, Rostron B, Cosgrove C, et al. Association of Cigarette, Cigar, and Pipe Use With Mortality Risk in the US Population. JAMA Intern Med. 2018;178(4):469-476.

25. Modesitt SC, Huang B, Shelton BJ, Wyatt S. Endometrial cancer in Kentucky: the impact of age, smoking status, and rural residence. Gynecol Oncol. 2006;103(1):300-306.

26. Wu Y, Sun W, Liu H, Zhang D. Age at Menopause and Risk of Developing Endometrial Cancer: A Meta-Analysis. Biomed Res Int. 2019;2019:8584130. 
27. Barry JA, Azizia MM, Hardiman PJ. Risk of endometrial, ovarian and breast cancer in women with polycystic ovary syndrome: a systematic review and meta-analysis. Hum Reprod Update. 2014;20(5):748-758.

28. Zhou Y, Wang X, Jiang Y, et al. Association between polycystic ovary syndrome and the risk of stroke and all-cause mortality: insights from a meta-analysis. Gynecol Endocrinol. 2017;33(12):904-910.

29. Gong TT, Wang YL, Ma XX. Age at menarche and endometrial cancer risk: a doseresponse meta-analysis of prospective studies. Sci Rep. 2015;5:14051.

30. Wu QJ, Li YY, Tu C, et al. Parity and endometrial cancer risk: a meta-analysis of epidemiological studies. Sci Rep. 2015;5:14243.

31. Albrektsen G, Heuch I, Wik E, Salvesen HB. Parity and time interval since childbirth influence survival in endometrial cancer patients. Int J Gynecol Cancer. 2009;19(4):665669.

32. Raglan O, Kalliala I, Markozannes G, et al. Risk factors for endometrial cancer: An umbrella review of the literature. Int J Cancer. 2019;145(7):1719-1730.

33. Sjogren LL, Morch LS, Lokkegaard E. Hormone replacement therapy and the risk of endometrial cancer: A systematic review. Maturitas. 2016;91:25-35.

34. Shim SH, Lee SJ, Kim SN. Effects of hormone replacement therapy on the rate of recurrence in endometrial cancer survivors: a meta-analysis. Eur J Cancer. 2014;50(9):1628-1637.

35. Felix AS, Arem H, Trabert B, et al. Menopausal hormone therapy and mortality among endometrial cancer patients in the NIH-AARP Diet and Health Study. Cancer Causes Control. 2015;26(8):1055-1063.

36. Saed L, Varse F, Baradaran HR, et al. The effect of diabetes on the risk of endometrial Cancer: an updated a systematic review and meta-analysis. BMC cancer. 2019;19(1):527.

37. Anastasi E, Filardi T, Tartaglione S, Lenzi A, Angeloni A, Morano S. Linking type 2 diabetes and gynecological cancer: an introductory overview. Clin Chem Lab Med. 2018;56(9):1413-1425.

38. Bjorge T, Stocks T, Lukanova A, et al. Metabolic syndrome and endometrial carcinoma. Am J Epidemiol. 2010;171(8):892-902.

39. Arthur RS, Kabat GC, Kim MY, et al. Metabolic syndrome and risk of endometrial cancer in postmenopausal women: a prospective study. Cancer Causes Control. 2019;30(4):355-363.

40. Zanders MM, Boll D, van Steenbergen LN, van de Poll-Franse LV, Haak HR. Effect of diabetes on endometrial cancer recurrence and survival. Maturitas. 2013;74(1):37-43. 
41. Nicholas Z, Hu N, Ying J, Soisson P, Dodson M, Gaffney DK. Impact of comorbid conditions on survival in endometrial cancer. Am J Clin Oncol. 2014;37(2):131-134.

42. Chia VM, Newcomb PA, Trentham-Dietz A, Hampton JM. Obesity, diabetes, and other factors in relation to survival after endometrial cancer diagnosis. Int J Gynecol Cancer. 2007;17(2):441-446.

43. Ward KK, Shah NR, Saenz CC, McHale MT, Alvarez EA, Plaxe SC. Cardiovascular disease is the leading cause of death among endometrial cancer patients. Gynecol Oncol. 2012;126(2):176-179.

44. Felix AS, Bower JK, Pfeiffer RM, Raman SV, Cohn DE, Sherman ME. High cardiovascular disease mortality after endometrial cancer diagnosis: Results from the Surveillance, Epidemiology, and End Results (SEER) Database. Int J Cancer. 2017;140(3):555-564.

45. Felix AS, Blair CK, Lehman A, et al. Cardiovascular disease mortality among women with endometrial cancer in the Iowa Women's Health Study. Cancer Causes Control. 2017;28(10):1043-1051.

46. Alberti KG, Eckel RH, Grundy SM, et al. Harmonizing the metabolic syndrome: a joint interim statement of the International Diabetes Federation Task Force on Epidemiology and Prevention; National Heart, Lung, and Blood Institute; American Heart Association; World Heart Federation; International Atherosclerosis Society; and International Association for the Study of Obesity. Circulation. 2009;120(16):1640-1645.

47. Saklayen MG. The Global Epidemic of the Metabolic Syndrome. Curr Hypertens Rep. 2018;20(2):12.

48. Riediger ND, Clara I. Prevalence of metabolic syndrome in the Canadian adult population. CMAJ. 2011;183(15):E1127-1134.

49. O'Neill S, O'Driscoll L. Metabolic syndrome: a closer look at the growing epidemic and its associated pathologies. Obes Rev. 2015;16(1):1-12.

50. Gathirua-Mwangi WG, Monahan PO, Murage MJ, Zhang J. Metabolic syndrome and total cancer mortality in the Third National Health and Nutrition Examination Survey. Cancer Causes Control. 2017;28(2):127-136.

51. Papatla K, Huang M, Slomovitz B. The obese endometrial cancer patient: how do we effectively improve morbidity and mortality in this patient population? Ann Oncol. 2016;27(11):1988-1994.

52. Esposito K, Chiodini P, Capuano A, Bellastella G, Maiorino MI, Giugliano D. Metabolic syndrome and endometrial cancer: a meta-analysis. Endocrine. 2014;45(1):28-36.

53. Stocks T, Bjorge T, Ulmer H, et al. Metabolic risk score and cancer risk: pooled analysis of seven cohorts. Int J Epidemiol. 2015;44(4):1353-1363. 
54. Gathirua-Mwangi WG, Song Y, Monahan PO, Champion VL, Zollinger TW. Associations of metabolic syndrome and C-reactive protein with mortality from total cancer, obesity-linked cancers and breast cancer among women in NHANES III. Int J Cancer. 2018;143(3):535-542.

55. Jin J, Dalwadi SM, Masand RP, Hall TR, Anderson ML, Ludwig MS. Association Between Metabolic Syndrome and Endometrial Cancer Survival in a SEER-Medicare Linked Database. Am J Clin Oncol. 2020;43(6):411-417.

56. Ni J, Zhu T, Zhao L, et al. Metabolic syndrome is an independent prognostic factor for endometrial adenocarcinoma. Clin Transl Oncol. 2015;17(10):835-839.

57. Shou H, Yan K, Song J, Zhao L, Zhang Y, Ni J. Metabolic syndrome affects the longterm survival of patients with non-endometrioid carcinoma of the uterine corpus. Int $J$ Gynaecol Obstet. 2020;148(1):96-101.

58. Friedenreich CM, Biel RK, Lau DC, et al. Case-control study of the metabolic syndrome and metabolic risk factors for endometrial cancer. Cancer Epidemiol Biomarkers Prev. 2011;20(11):2384-2395.

59. Arem H, Chlebowski R, Stefanick ML, et al. Body mass index, physical activity, and survival after endometrial cancer diagnosis: results from the Women's Health Initiative. Gynecol Oncol. 2013;128(2):181-186.

60. Arem H, Park Y, Pelser C, et al. Prediagnosis body mass index, physical activity, and mortality in endometrial cancer patients. J Natl Cancer Inst. 2013;105(5):342-349.

61. Arem H, Pfeiffer RM, Moore SC, Brinton LA, Matthews CE. Body mass index, physical activity, and television time in relation to mortality risk among endometrial cancer survivors in the NIH-AARP Diet and Health Study cohort. Cancer Causes Control. 2016;27(11):1403-1409.

62. Arem H, Pfeiffer RM, Moore SC, et al. Post-diagnosis body mass index and mortality among women diagnosed with endometrial cancer: Results from the Women's Health Initiative. PLoS One. 2017;12(2):e0171250.

63. Akbayir O, Corbacioglu Esmer A, Numanoglu C, et al. Influence of body mass index on clinicopathologic features, surgical morbidity and outcome in patients with endometrial cancer. Arch Gynecol Obstet. 2012;286(5):1269-1276.

64. Benedetti Panici P, Basile S, Salerno MG, et al. Secondary analyses from a randomized clinical trial: age as the key prognostic factor in endometrial carcinoma. Am J Obstet Gynecol. 2014;210(4):363 e361-363 e310.

65. Blackburn BE, Soisson S, Rowe K, et al. Prognostic factors for rural endometrial cancer patients in a population-based cohort. BMC Public Health. 2019;19(1):921. 
66. Calle EE, Rodriguez C, Walker-Thurmond K, Thun MJ. Overweight, Obesity, and Mortality from Cancer in a Prospectively Studied Cohort of U.S. Adults. N Engl J Med. 2003.

67. Chaves GV, de Almeida Simao T, Pinto LFR, Moreira MAM, Bergmann A, Chaves CBP. Overweight and obesity do not determine worst prognosis in endometrioid endometrial carcinoma. Arch Gynecol Obstet. 2019;300(6):1671-1677.

68. Canlorbe G, Bendifallah S, Raimond E, et al. Severe Obesity Impacts Recurrence-Free Survival of Women with High-Risk Endometrial Cancer: Results of a French Multicenter Study. Ann Surg Oncol. 2015;22(8):2714-2721.

69. Crosbie EJ, Roberts C, Qian W, Swart AM, Kitchener HC, Renehan AG. Body mass index does not influence post-treatment survival in early stage endometrial cancer: results from the MRC ASTEC trial. Eur J Cancer. 2012;48(6):853-864.

70. Edlinger M, Concin N, Concin H, Nagel G, Ulmer H, Gobel G. Lifestyle-related biomarkers and endometrial cancer survival: elevated gamma-glutamyltransferase as an important risk factor. Cancer Epidemiol. 2013;37(2):156-161.

71. Felix AS, Scott McMeekin D, Mutch D, et al. Associations between etiologic factors and mortality after endometrial cancer diagnosis: the NRG Oncology/Gynecologic Oncology Group 210 trial. Gynecol Oncol. 2015;139(1):70-76.

72. Felix AS, Brasky TM, Cohn DE, et al. Endometrial carcinoma recurrence according to race and ethnicity: An NRG Oncology/Gynecologic Oncology Group 210 Study. Int J Cancer. 2018;142(6):1102-1115.

73. Gates EJ, Hirschfield L, Matthews RP, Yap OW. Body mass index as a prognostic factor in endometrioid adenocarcinoma of the endometrium. J Natl Med Assoc. 2006;98(11):1814-1822.

74. Gillen J, Mills KA, Dvorak J, et al. Imaging biomarkers of adiposity and sarcopenia as potential predictors for overall survival among patients with endometrial cancer treated with bevacizumab. Gynecol Oncol Rep. 2019;30:100502.

75. Giannini A, Di Donato V, Schiavi MC, May J, Panici PB, Congiu MA. Predictors of postoperative overall and severe complications after surgical treatment for endometrial cancer: The role of the fragility index. Int J Gynaecol Obstet. 2020;148(2):174-180.

76. Hein A, Schneider MO, Renner SK, et al. Risk of postmenopausal hormone therapy and patient history factors for the survival rate in women with endometrial carcinoma. Arch Gynecol Obstet. 2020;301(1):289-294.

77. Khan M, Mori M, Sakauchi F, et al. Risk of endometrial cancer mortality by ever-use of sex hormones and other factors in Japan. Asian Pac J Cancer Prev. 2006;7(2):260-266. 
78. Ko EM, Walter P, Clark L, et al. The complex triad of obesity, diabetes and race in Type I and II endometrial cancers: prevalence and prognostic significance. Gynecol Oncol. 2014;133(1):28-32.

79. Lindemann K, Cvancarova M, Eskild A. Body mass index, diabetes and survival after diagnosis of endometrial cancer: A report from the HUNT-Survey. Gynecol Oncol. 2015;139(3):476-480.

80. Lemanska A, Zaborowski M, Spaczynski M, Nowak-Markwitz E. Do endometrial cancer patients benefit from metformin intake? Ginekol Pol. 2015;86(6):419-423.

81. Linkov F, Taioli E. Factors influencing endometrial cancer mortality: the Western Pennsylvania Registry. Future Oncol. 2008;4(6):857-865.

82. Lee SH, Park J-W, Han M, Cho YJ. Lymphovascular space invasion, a prognostic marker for disease recurrence in patients with early endometrioid endometrial cancer. Current Gynecologic Oncology. 2017;15(3):183-188.

83. Lutman CV, Havrilesky LJ, Cragun JM, et al. Pelvic lymph node count is an important prognostic variable for FIGO stage I and II endometrial carcinoma with high-risk histology. Gynecol Oncol. 2006;102(1):92-97.

84. Matsuo K, Moeini A, Cahoon SS, et al. Weight Change Pattern and Survival Outcome of Women with Endometrial Cancer. Ann Surg Oncol. 2016;23(9):2988-2997.

85. Mauland KK, Trovik J, Wik E, et al. High BMI is significantly associated with positive progesterone receptor status and clinico-pathological markers for non-aggressive disease in endometrial cancer. Br J Cancer. 2011;104(6):921-926.

86. Modesitt SC, Tian C, Kryscio R, et al. Impact of body mass index on treatment outcomes in endometrial cancer patients receiving doxorubicin and cisplatin: a Gynecologic Oncology Group study. Gynecol Oncol. 2007;105(1):59-65.

87. Nattenmüller J, Rom J, Buckner T, et al. Visceral abdominal fat measured by computer tomography as a prognostic factor for gynecological malignancies? Oncotarget. 2018;9(23).

88. Ruterbusch JJ, Ali-Fehmi R, Olson SH, et al. The influence of comorbid conditions on racial disparities in endometrial cancer survival. Am J Obstet Gynecol. 2014;211(6):627 e621-629.

89. Todo Y, Okamoto K, Minobe S, Kato H. Clinical significance of surgical staging for obese women with endometrial cancer: a retrospective analysis in a Japanese cohort. Jpn J Clin Oncol. 2014;44(10):903-909.

90. Tuomi T, Pasanen A, Leminen A, Butzow R, Loukovaara M. Prediction of Site-Specific Tumor Relapses in Patients With Stage I-II Endometrioid Endometrial Cancer. Int J Gynecol Cancer. 2017;27(5):923-930. 
91. Van Arsdale A, Miller DT, Kuo DY, Isani S, Sanchez L, Nevadunsky NS. Association of obesity with survival in patients with endometrial cancer. Gynecol Oncol. 2019;154(1):156-162.

92. von Gruenigen VE, Tian C, Frasure H, Waggoner S, Keys H, Barakat RR. Treatment effects, disease recurrence, and survival in obese women with early endometrial carcinoma : a Gynecologic Oncology Group study. Cancer. 2006;107(12):2786-2791.

93. Jeong NH, Lee JM, Lee JK, et al. Role of body mass index as a risk and prognostic factor of endometrioid uterine cancer in Korean women. Gynecol Oncol. 2010;118(1):24-28.

94. Kristensen AB, Hare-Bruun H, Hogdall CK, Rudnicki M. Influence of Body Mass Index on Tumor Pathology and Survival in Uterine Cancer: A Danish Register Study. Int J Gynecol Cancer. 2017;27(2):281-288.

95. Olson SH, Atoria CL, Cote ML, et al. The impact of race and comorbidity on survival in endometrial cancer. Cancer Epidemiol Biomarkers Prev. 2012;21(5):753-760.

96. Temkin SM, Pezzullo JC, Hellmann M, Lee YC, Abulafia O. Is body mass index an independent risk factor of survival among patients with endometrial cancer? Am J Clin Oncol. 2007;30(1):8-14.

97. Reeves GK, Pirie K, Beral V, et al. Cancer incidence and mortality in relation to body mass index in the Million Women Study: cohort study. BMJ. 2007;335(7630):1134.

98. Martra F, Kunos C, Gibbons H, et al. Adjuvant treatment and survival in obese women with endometrial cancer: an international collaborative study. Am J Obstet Gynecol. 2008;198(1):89 e81-88.

99. Secord AA, Hasselblad V, Von Gruenigen VE, et al. Body mass index and mortality in endometrial cancer: A systematic review and meta-analysis. Gynecol Oncol. 2016;140(1):184-190.

100. Al Hilli MM, Bakkum-Gamez JN, Mariani A, et al. The effect of diabetes and metformin on clinical outcomes is negligible in risk-adjusted endometrial cancer cohorts. Gynecol Oncol. 2016;140(2):270-276.

101. Boll D, Verhoeven RH, van der Aa MA, Lybeert ML, Coebergh JW, Janssen-Heijnen ML. Adherence to national guidelines for treatment and outcome of endometrial cancer stage I in relation to co-morbidity in southern Netherlands 1995-2008. Eur J Cancer. 2011;47(10):1504-1510.

102. Burghaus S, Halmen S, Gass P, et al. Outcome and prognosis in uterine sarcoma and malignant mixed Mullerian tumor. Arch Gynecol Obstet. 2016;294(2):343-351.

103. Campbell PT, Newton CC, Patel AV, Jacobs EJ, Gapstur SM. Diabetes and causespecific mortality in a prospective cohort of one million U.S. adults. Diabetes Care. 2012;35(9):1835-1844. 
104. Chen Y, Wu F, Saito E, et al. Association between type 2 diabetes and risk of cancer mortality: a pooled analysis of over 771,000 individuals in the Asia Cohort Consortium. Diabetologia. 2017;60(6):1022-1032.

105. Folsom AR, Anderson KE, Sweeney C, Jacobs DR, Jr. Diabetes as a risk factor for death following endometrial cancer. Gynecol Oncol. 2004;94(3):740-745.

106. Larouzee E, Phelippeau J, Roberti E, Koskas M. Evaluation of the French medical practices in endometrial cancer management by using quality indicators. Eur J Obstet Gynecol Reprod Biol. 2019;236:198-204.

107. Lam C, Cronin K, Ballard R, Mariotto A. Differences in cancer survival among white and black cancer patients by presence of diabetes mellitus: Estimations based on SEERMedicare-linked data resource. Cancer Med. 2018.

108. Ranc K, Jorgensen ME, Friis S, Carstensen B. Mortality after cancer among patients with diabetes mellitus: effect of diabetes duration and treatment. Diabetologia. 2014;57(5):927-934.

109. Wang Y, Garabedian MJ, Logan SK. URI1 amplification in uterine carcinosarcoma associates with chemo-resistance and poor prognosis. Am J Cancer Res. 2015;5(7):23202329.

110. Feinberg J, Albright B, Black J, et al. Ten-Year Comparison Study of Type 1 and 2 Endometrial Cancers: Risk Factors and Outcomes. Gynecol Obstet Invest. 2019;84(3):290-297.

111. Fleming ND, Lentz SE, Cass I, Li AJ, Karlan BY, Walsh CS. Is older age a poor prognostic factor in stage I and II endometrioid endometrial adenocarcinoma? Gynecol Oncol. 2011;120(2):189-192.

112. Neeland IJ, Ross R, Despres JP, et al. Visceral and ectopic fat, atherosclerosis, and cardiometabolic disease: a position statement. Lancet Diabetes Endocrinol. 2019;7(9):715-725.

113. Friedenreich CM, Cook LS, Magliocco AM, Duggan MA, Courneya KS. Case-control study of lifetime total physical activity and endometrial cancer risk. Cancer Causes Control. 2010;21(7):1105-1116.

114. North American Association of Cancer Registries. Cancer in North America 1991-1995. Sacramento, CA1999.

115. Friedenreich CM, Courneya KS, Bryant HE. The lifetime total physical activity questionnaire: development and reliability. Med Sci Sports Exerc. 1998;30(2):266-274.

116. Csizmadi I, Kahle L, Ullman R, et al. Adaptation and evaluation of the National Cancer Institute's Diet History Questionnaire and nutrient database for Canadian populations. Public Health Nutr. 2007;10(1):88-96. 
117. Willis G. Cognitive interviewing and questionnaire design: A training manual. . Natl Cent Heal Stat Cogn Methods Staff Work Pap Ser. 1994.

118. American Joint Committee on Cancer AJCC. AJCC Cancer Staging Manual. 5th ed. Philadelphia, PA: Lippincott-Raven; 1997.

119. Adambekov S, Yi Y, Fabio A, et al. Metabolic Syndrome in Endometrial Cancer Patients: Systematic Review. Metab Syndr Relat Disord. 2019;17(5):241-249.

120. Amankwah EK, Friedenreich CM, Magliocco AM, et al. Anthropometric measures and the risk of endometrial cancer, overall and by tumor microsatellite status and histological subtype. Am J Epidemiol. 2013;177(12):1378-1387.

121. van Weelden WJ, Fasmer KE, Tangen IL, et al. Impact of body mass index and fat distribution on sex steroid levels in endometrial carcinoma: a retrospective study. $B M C$ cancer. 2019;19(1):547.

122. Zhang C, Rexrode KM, van Dam RM, Li TY, Hu FB. Abdominal obesity and the risk of all-cause, cardiovascular, and cancer mortality: sixteen years of follow-up in US women. Circulation. 2008;117(13):1658-1667.

123. Lugo D, Pulido AL, Mihos CG, et al. The effects of physical activity on cancer prevention, treatment and prognosis: A review of the literature. Complement Ther Med. 2019;44:9-13.

124. Vishnu A, Gurka MJ, DeBoer MD. The severity of the metabolic syndrome increases over time within individuals, independent of baseline metabolic syndrome status and medication use: The Atherosclerosis Risk in Communities Study. Atherosclerosis. 2015;243(1):278-285.

125. Diabetes Canada Clinical Practice Guidelines Expert Committee, Punthakee Z, Goldenberg R, Katz P. Definition, Classification and Diagnosis of Diabetes, Prediabetes and Metabolic Syndrome. Can J Diabetes. 2018;42 Suppl 1:S10-S15.

126. Yang X, Wang J. The Role of Metabolic Syndrome in Endometrial Cancer: A Review. Front Oncol. 2019;9:744.

127. Pirro M, Ricciuti B, Rader DJ, Catapano AL, Sahebkar A, Banach M. High density lipoprotein cholesterol and cancer: Marker or causative? Prog Lipid Res. 2018;71:54-69.

128. Tucker K, Staley SA, Clark LH, Soper JT. Physical Activity: Impact on Survival in Gynecologic Cancer. Obstet Gynecol Surv. 2019;74(11):679-692.

129. Mongraw-Chaffin M, Foster MC, Anderson CAM, et al. Metabolically Healthy Obesity, Transition to Metabolic Syndrome, and Cardiovascular Risk. J Am Coll Cardiol. 2018;71(17):1857-1865. 
130. Lavie CJ, Ozemek C, Carbone S, Katzmarzyk PT, Blair SN. Sedentary Behavior, Exercise, and Cardiovascular Health. Circ Res. 2019;124(5):799-815.

131. Amant F, Mirza MR, Koskas M, Creutzberg CL. Cancer of the corpus uteri. Int J Gynaecol Obstet. 2018;143 Suppl 2:37-50.

132. Patten SB. Epidemiology for Canadian Students, 2nd ed.: Principles, Methods and Critical Appraisal. Canada: Brush Education; 2018.

133. Ross R, Neeland IJ, Yamashita S, et al. Waist circumference as a vital sign in clinical practice: a Consensus Statement from the IAS and ICCR Working Group on Visceral Obesity. Nature reviews Endocrinology. 2020;16(3):177-189.

134. American Diabetes Association. 2. Classification and Diagnosis of Diabetes: Standards of Medical Care in Diabetes-2020. Diabetes Care. 2020;43(Suppl 1):S14-S31.

135. Joint British Societies' Board. Joint British Societies' consensus recommendations for the prevention of cardiovascular disease (JBS3). Heart. 2014;100 Suppl 2:ii1-ii67.

136. Yeganeh L, Harrison C, Vincent AJ, Teede H, Boyle JA. Effects of lifestyle modification on cancer recurrence, overall survival and quality of life in gynaecological cancer survivors: A systematic review and meta-analysis. Maturitas. 2018;111:82-89.

137. Tollosa DN, Holliday E, Hure A, Tavener M, James EL. Multiple health behaviors before and after a cancer diagnosis among women: A repeated cross-sectional analysis over 15 years. Cancer Med. 2020;9(9):3224-3233.

138. von Gruenigen V, Frasure H, Kavanagh MB, et al. Survivors of uterine cancer empowered by exercise and healthy diet (SUCCEED): a randomized controlled trial. Gynecol Oncol. 2012;125(3):699-704. 


\section{APPENDIX A}

Literature Review Search Strategies

EMBASE (Ovid) databases 1990-March 19, 2020

1. uterus cancer/

2. endometrium tumor/

3. endometrium cancer/

4. (endometr* adj4 canc*).tw,kw.

5. (endometr* adj4 carcinom*).tw,kw.

6. (uter* adj4 canc*).tw,kw.

7. (uter* adj4 neopla*).tw,kw.

8. (endometr* adj4 neopla*).tw,kw.

9. 1 or 2 or 3 or 4 or 5 or 6 or 7 or 8

10. vital statistics/

11. mortality/

12. survival rate/

13. disease free survival/

14. survival analysis/

15. progression free survival/

16. tumor recurrence/

17. recurrent disease/

18. cancer mortality/

19. "cancer survival"/

20. Cause of Death/

21. Surviv*.tw,kw.

22. mortal*.tw,kw.

23. fatal.tw,kw.

24. Death.tw,kw.

25. Overall surviv*.tw,kw.

26. vital statist*.tw,kw.

27. (surviv* adj4 analys*).tw,kw.

28. (Neoplas* adj4 Recurren*).tw,kw.

29. (canc* adj4 Recurren*).tw,kw.

30. Recurren*.tw,kw.

31. (mort* adj4 endomet*).tw,kw.

32. (Cause adj4 Death).tw,kw.

33. 10 or 11 or 12 or 13 or 14 or 15 or 16 or 17 or 18 or 19 or 20 or 21 or 22 or 23 or 24 or 25 or 26 or 27 or 28 or 29 or 30 or 31 or 32

34.9 and 33

35. "metabolic syndrome".tw,kw.

36. obesity/ 
37. "disorders of lipid and lipoprotein metabolism"/

38. metabolic syndrome $\mathrm{x} /$

39. body mass/

40. morphometry/

41. anthropometry/

42. adipose tissue/

43. BMI.tw,kw.

44. Obes*.tw,kw.

45. Body mass index.tw,kw.

46. diabetes mellitus/

47. diabet*.tw,kw.

48. high density lipoprotein cholesterol/

49. dyslipidemia/

50. triacylglycerol/

51. glucose level/ or glucose blood level/

52. hypertension/ or elevated blood pressure/

53. hypertension.tw,kw.

54. HDL.tw,kw.

55. waist circumference/

56. Waist circumference.tw,kw.

57. triglycerid*.tw,kw.

58. cholester*.tw,kw.

59. "blood press*".tw,kw.

60.35 or 36 or 37 or 38 or 39 or 40 or 41 or 42 or 43 or 44 or 45 or 46 or 47 or 48 or 49 or 50 or 51 or 52 or 53 or 54 or 55 or 56 or 57 or 58 or 59

61.34 and 60

62. limit 61 to $\mathrm{yr}=" 1990$-Current"

\section{MEDLINE (Ovid) databases 1990-March 19, 2020}

1. uterus cancer/

2. Endometrial Neoplasms/

3. (endometr* adj4 canc*).tw,kf.

4. (endometr* adj4 carcinom*).tw,kf.

5. (uter* adj4 canc*).tw,kf.

6. (uter* adj4 neopla*).tw,kf.

7. (endometr* adj4 neopla*).tw,kf.

8.1 or 2 or 3 or 4 or 5 or 6 or 7

9. vital statistics/

10. mortality/

11. survival rate/ 
12. disease-free survival/

13. progression-free survival/

14. survival analysis/

15. Neoplasm Recurrence, Local/

16. Recurrence/

17. Endometrial Neoplasms/mo [Mortality]

18. Cause of Death/

19. Surviv*.tw,kf.

20. morta*.tw,kf.

21. Fatal.tw,kf.

22. Death.tw,kf.

23. Overall surviv*.tw,kf.

24. vital statist*.tw,kf.

25. (surviv* adj4 rate).tw,kf.

26. (surviv* adj4 analys*).tw,kf.

27. (canc* adj4 Recurren*).tw,kf.

28. Recurren*.tw,kf.

29. (mort* adj4 endomet*).tw,kf.

30. (Cause adj4 Death).tw,kf.

31.9 or 10 or 11 or 12 or 13 or 14 or 15 or 16 or 17 or 18 or 19 or 20 or 21 or 22 or 23 or 24 or 25 or 26 or 27 or 28 or 29 or 30

32.8 and 31

33. "metabolic syndrome".tw,kf.

34. metabolic syndrome/ or metabolic diseases/

35. glucose metabolism disorders/

36. diabetes mellitus/

37. hyperglycemia/

38. hyperinsulinism/

39. insulin resistance/

40. lipid metabolism disorders/

41. dyslipidemias/

42. obesity/

43. "body weights and measures"/

44. body mass index/

45. anthropometry/

46. Adipose Tissue/

47. Body mass index.tw,kf.

48. BMI.tw,kf.

49. Obes*.tw,kf.

50. Waist circumference.tw,kf.

51. diabet*.tw,kf. 
52. cholesterol/ or cholesterol, hdl/ or lipoproteins, hdl/

53. HDL.tw,kf.

54. waist circumference/

55. Dyslipidemias/

56. diabetes mellitus, type 2/ or hyperglycemia/ or hyperinsulinism/

57. hypertension.tw,kf.

58. Hypertension/

59. triglycerid*.tw,kf.

60. "blood press*".tw,kf.

61.33 or 34 or 35 or 36 or 37 or 38 or 39 or 40 or 41 or 42 or 43 or 44 or 45 or 46 or 47 or 48 or 49 or 50 or 51 or 52 or 53 or 54 or 55 or 56 or 57 or 58 or 59 or 60

62.32 and 61

63. limit 62 to $\mathrm{yr}=" 1990-2020 "$ 


\section{APPENDIX B}

\section{Letter of Permission}

I, Christine Friedenreich on June 25, 2020 grant permission to include the below mentioned manuscript in Renee Kokts-Porietis Masters Thesis “A Prospective Cohort Study of Metabolic Syndrome and Endometrial Cancer Survival."

Citation of manuscript included in thesis:

Kokts-Porietis RL, McNeil J, Nelson GS, Courneya KS, Cook LS, Friedenreich CM. Prospective Cohort Study of Metabolic Syndrome and Endometrial Cancer Survival. Gynecologic Oncology, in press.

This thesis will be added to the institutional repository at the University of Calgary and the Library and Archives Canada.

University of Calgary Theses Repository - The Vault http://theses.ucalgary.ca/

Library and Archives Canada http://collectionscanada.gc.ca/obj/s4/f2/frm-nl59-2-e.pdf 


\section{Letter of Permission}

I_Gregg Nelson__ on June_25_2020 grant permission to include the below mentioned manuscript in Renee Kokts-Porietis Masters Thesis “A Prospective Cohort Study of Metabolic Syndrome and Endometrial Cancer Survival.”

Citation of manuscript included in thesis:

Kokts-Porietis RL, McNeil J, Nelson GS, Courneya KS, Cook LS, Friedenreich CM. Prospective Cohort Study of Metabolic Syndrome and Endometrial Cancer Survival. Gynecologic Oncology, in press.

This thesis will be added to the institutional repository at the University of Calgary and the Library and Archives Canada.

University of Calgary Theses Repository - The Vault http://theses.ucalgary.ca/

Library and Archives Canada http://collectionscanada.gc.ca/obj/s4/f2/frm-nl59-2-e.pdf 


\section{Letter of Permission}

I, Linda S. Cook, on June 25, 2020 grant permission to include the below mentioned manuscript in Renee Kokts-Porietis Masters Thesis "A Prospective Cohort Study of Metabolic Syndrome and Endometrial Cancer Survival.”

Citation of manuscript included in thesis:

Kokts-Porietis RL, McNeil J, Nelson GS, Courneya KS, Cook LS, Friedenreich CM. Prospective Cohort Study of Metabolic Syndrome and Endometrial Cancer Survival. Gynecologic Oncology, in press.

This thesis will be added to the institutional repository at the University of Calgary and the Library and Archives Canada.

University of Calgary Theses Repository - The Vault http://theses.ucalgary.ca/

Library and Archives Canada http://collectionscanada.gc.ca/obj/s4/f2/frm-nl59-2-e.pdf 


\section{Letter of Permission}

I, Jessica McNeil, on June 252020 grant permission to include the below mentioned manuscript in Renee Kokts-Porietis Masters Thesis "A Prospective Cohort Study of Metabolic Syndrome and Endometrial Cancer Survival.”

Citation of manuscript included in thesis:

Kokts-Porietis RL, McNeil J, Nelson GS, Courneya KS, Cook LS, Friedenreich CM. Prospective Cohort Study of Metabolic Syndrome and Endometrial Cancer Survival. Gynecologic Oncology, in press.

This thesis will be added to the institutional repository at the University of Calgary and the Library and Archives Canada.

University of Calgary Theses Repository - The Vault http://theses.ucalgary.ca/

Library and Archives Canada http://collectionscanada.gc.ca/obj/s4/f2/frm-nl59-2-e.pdf 


\section{Letter of Permission}

I_Kerry Courneya , on June _26 2020 grant permission to include the below mentioned manuscript in Renee Kokts-Porietis Masters Thesis "A Prospective Cohort Study of Metabolic Syndrome and Endometrial Cancer Survival.”

Citation of manuscript included in thesis:

Kokts-Porietis RL, McNeil J, Nelson GS, Courneya KS, Cook LS, Friedenreich CM. Prospective Cohort Study of Metabolic Syndrome and Endometrial Cancer Survival. Gynecologic Oncology, in press.

This thesis will be added to the institutional repository at the University of Calgary and the Library and Archives Canada.

University of Calgary Theses Repository - The Vault http://theses.ucalgary.ca/

Library and Archives Canada http://collectionscanada.gc.ca/obj/s4/f2/frm-nl59-2-e.pdf 